\title{
IMPROVING DOMESTIC SPACE HEATING: A COST BENEFIT ANALYSIS BASED ON A RANDOMISED COMMUNITY TRIAL
}

\author{
by \\ Nicholas Jonathan Preval \\ Thesis \\ ENVIRONMENTAL STUDIES 593 \\ 2008 \\ A 90 point thesis submitted to Victoria University of Wellington,
} as partial fulfilment for the degree of Master of Environmental Studies.

School of Geography, Environment and Earth Sciences

Victoria University of Wellington

February 2008 


\section{ACKNOWLEDGEMENTS}

I would like to extend my heartfelt thanks to the many people who made it possible for me to complete my research. To my supervisor, Associate Professor Ralph Chapman, who patiently guided me and provided invaluable support in conceptualising, proof-reading and writing this thesis. To Professor Philippa HowdenChapman, Nevil Pierse, Sarah Nicholls, Julie Gillespie-Bennett, Dr. Malcolm Cunningham, Dr. Robyn Phipps, Mikael Boulic, Dr. Pär Fjällström, Sarah Free, Associate Professor Bob Lloyd, Helen Viggers, Dr. Kristin Wickens, David Shields, Associate Professor Michael Baker, Professor Chris Cunningham, Professor Alistair Woodward, Dr. Chris Bullen, Professor Julian Crane and all of the other members of He Kainga Oranga, who carried out the research that is the basis of my thesis and who provided a great deal of support, advice and encouragement. Particular thanks to Nevil Pierse, who gave a great deal of his time in helping me to understand the data and to analyse them appropriately.

I acknowledge the funders of the Housing, Heating and Health Study; Health Research Council of New Zealand, Contact Energy, Ministry for the Environment, Hutt Valley DHB, EECA, Housing New Zealand Corporation, Capital and Coast District Health Board, LPG Association, Otago University, Massey University and Building Research, without whom the valuable research that this thesis is based on could not have occurred.

Thanks to the children and their families/whanau who took part in the Study, the community coordinators, interviewers, general practitioners, energy companies, retrofitters and heater suppliers.

Thanks also to the faculty and students of the School of Geography, Environment and Earth Sciences, who provided a nurturing and challenging academic environment.

On a more personal level, I would like to thank my partner Mary-Lou Harris - I would not have completed my thesis without your unflagging support. I would also 
like to express my appreciation for the love and encouragement of my family. Thanks also to my classmates, particularly Tush and Patrick who stopped me from taking my thesis, or anything else, too seriously. 


\begin{abstract}
Despite New Zealand's temperate climate, New Zealand homes are generally cold, primarily as the result of a historical lack of insulation. Many New Zealand households also suffer fuel poverty and have inadequate domestic space heating, including unflued gas heaters which emit harmful gases directly into the indoor environment.
\end{abstract}

There is a large body of evidence correlating improved domestic space heating and respiratory health outcomes such as asthma. There is also evidence of connections between improved domestic space heating and mental health, COPD, rheumatism, ischaemic heart disease and strokes. Improvements in domestic space heating have the potential to improve occupant health via increased temperatures and reduced dampness, mould, and harmful emissions and also have the potential to reduce household energy bills and $\mathrm{CO}_{2}$ emissions.

This potential was the basis of the Housing, Heating and Health Study, a randomised community trial carried out by He Kainga Oranga, the Housing and Health Research Programme of the School of Medicine and Health Sciences of the University of Otago, Wellington, which involved the installation of energy efficient and healthy heaters in the dwellings of families who used ineffective heating and included an asthmatic child aged seven to twelve.

This thesis is a cost benefit analysis based primarily on energy use and health outcome related data from the Housing, Heating and Health Study. It concludes that the outcome of the intervention was equivocal from a societal perspective, due in part to limitations of the data and analysis, with a negative "net present value" (NPV) for the baseline scenario, but positive NPVs for a number of alternative scenarios and a strong suggestion that if the full benefits of the intervention were captured that the NPV of the intervention is likely to be positive. Predicted changes to the New Zealand economy resulting from climate change mitigation policies and increasing real energy costs also increase the likelihood that similar future interventions may have a positive NPV. 


\section{TABLE OF CONTENTS}

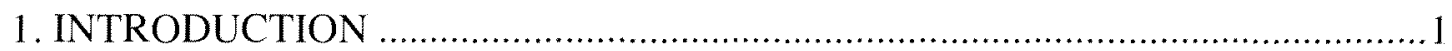

1.1 The Significance of Domestic Space Heating ................................................

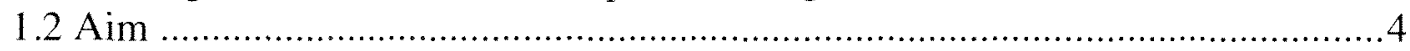

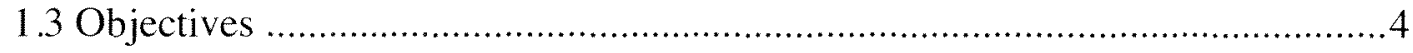

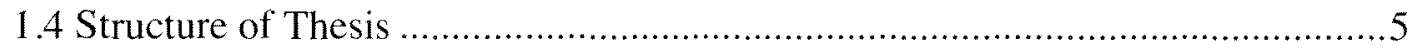

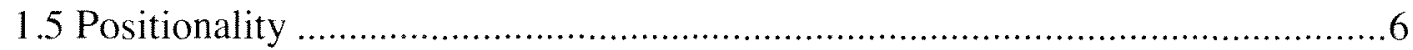

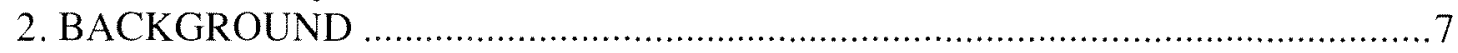

2.1 Housing and Heating in New Zealand ......................................................... 7

2.1.1 Energy and domestic space heating in New Zealand ..............................9

2.2 Connections between Housing, Heating and Health .................................. 10

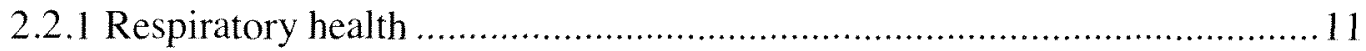

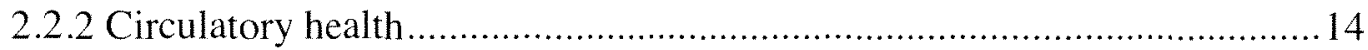

2.2.3 Other potential health effects .......................................................... 15

2.2.4 Mental health ............................................................................. 16

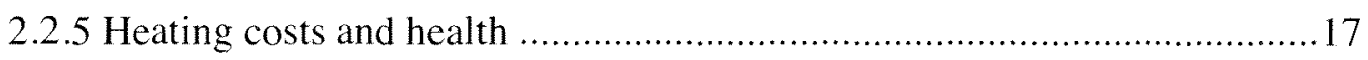

2.3 Housing Interventions and Health ............................................................ 18

2.3.1 The Housing, Heating and Health Study .............................................20

2.3.2 Data from the Housing, Heating and Health Study ................................22

2.4 Economic Analysis of Housing, Heating and Health ...................................23

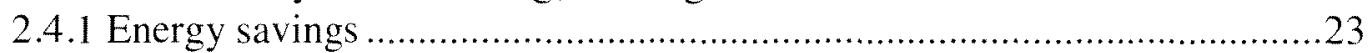

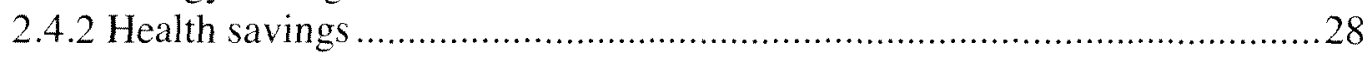

2.4.3 Comprehensive cost benefit analyses of housing improvements ..............29

2.4.4 Cost benefit analysis of the Housing, Insulation and Health Study ............31

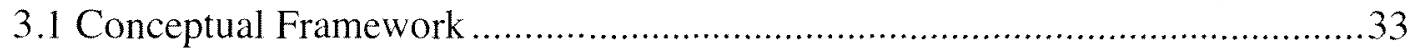

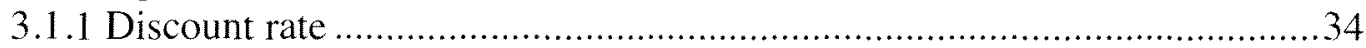

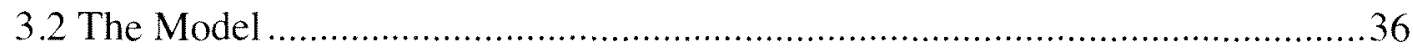

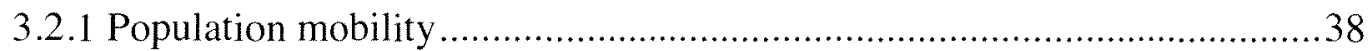

3.2.2 Prevalence of asthma in New Zealand ...............................................40

3.2.3 Purchase, maintenance and replacement of domestic space heating ..........42

3.2.4 Extrapolating winter data .............................................................4. 48

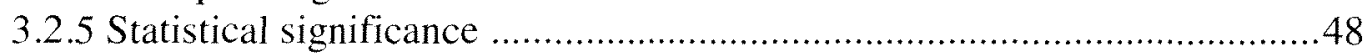

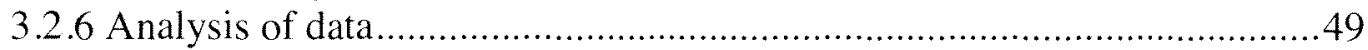

3.3 Analysis of Available Data from the Housing, Heating and Health Study .......50

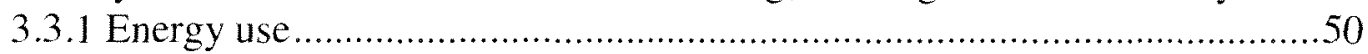

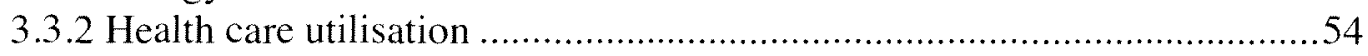

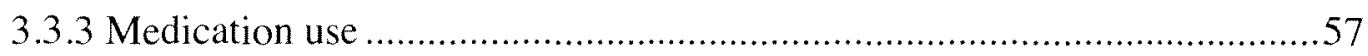

3.3.4 Days off school/paid work due to illness ..........................................58

3.4 Issues beyond the Direct Scope of the Data.................................................60

3.4.1 The value of improved comfort and mental health ..............................60

3.4.2 The value of new cases of asthma avoided ...........................................63

3.4.3 Excess mortality avoided .....................................................................63

3.4.4 The value of caregiver days off work ...............................................64

3.4.5 The value of reduced damp and mould related repair costs....................65

3.4.6 The potential impact of summer air conditioning using heat pumps .........65

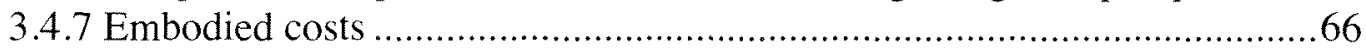

3.4.8 Non $\mathrm{CO}_{2}$ external emissions from electricity generation ......................66 
4.1 Cost of Heater Purchase, Maintenance and Replacement 69

4.2 Benefits of Changes in Health Care Utilisation, Medication Use and Days off

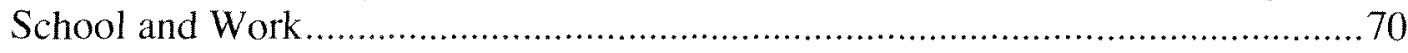

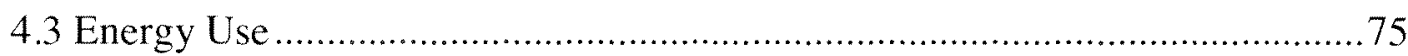

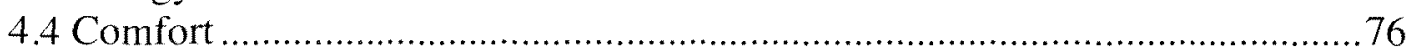

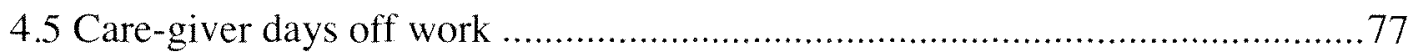

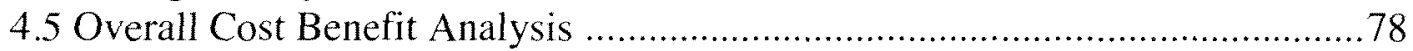

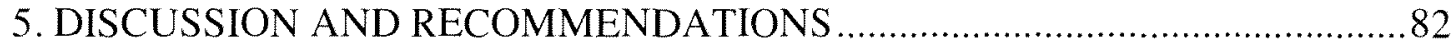

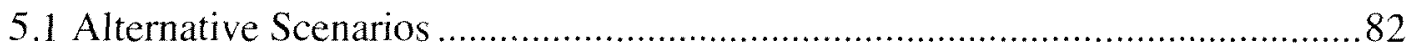

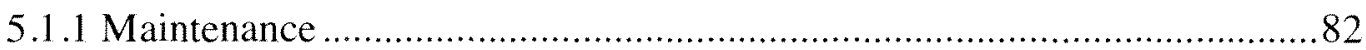

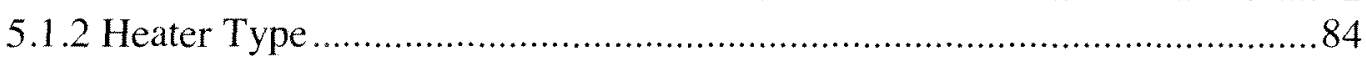

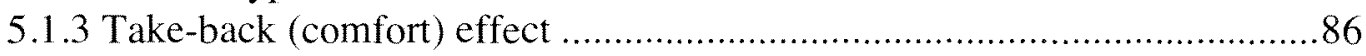

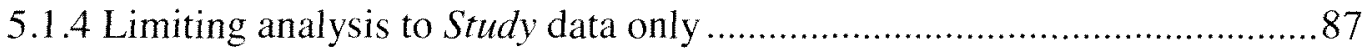

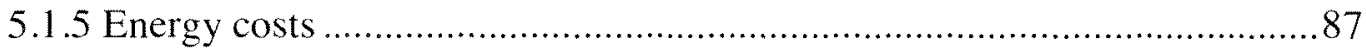

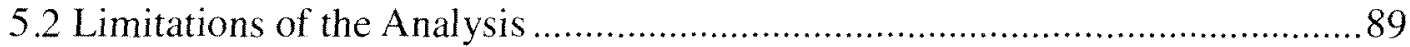

5.3 The Research and Policy Context ........................................................... 91

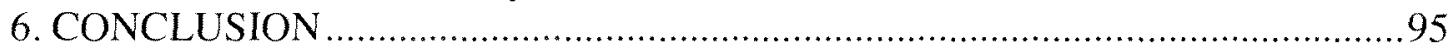

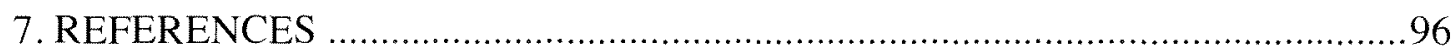




\section{LIST OF FIGURES}

Figure 1: An Example of the Calculation of the NPV of the Intervention per Household .50

Figure 2: Key .69 


\section{LIST OF TABLES}

Table 1: Housing, Insulation and Health Study: Costs and Benefits per Household ..31

Table 2: Household Composition ...................................................................42

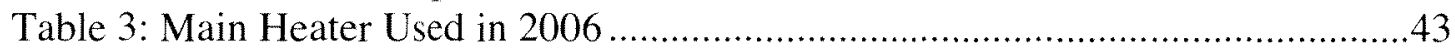

Table 4: Study Heaters Installed in Intervention Group Households ........................44

Table 5: Heater Installation Cost and Average Life-span ......................................45

Table 6: Annual Maintenance Costs ........................................................................46

Table 7: Energy Use Costs and Emissions per Unit .............................................52

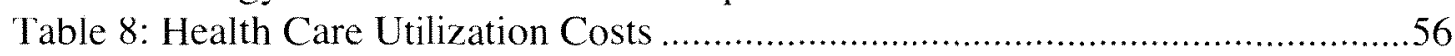

Table 9: Health Cost of Non- $\mathrm{CO}_{2}$ External Emissions from Electricity Generation in

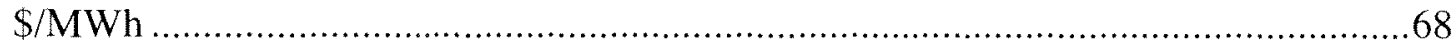

Table 10: NPV of Change in Heater Purchasing, Maintenance and Replacement Costs per Intervention Group Household Following the Intervention ................................70

Table 11: Value of Health Outcomes per Asthmatic Child as a Result of the Intervention ............................................................................................... 71

Table 12: Value of Health Outcomes per Non-asthmatic Child as a Result of the

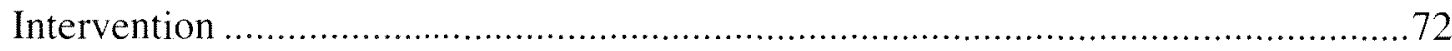

Table 13: Value of Health Outcomes per Asthmatic Adult as a Result of the

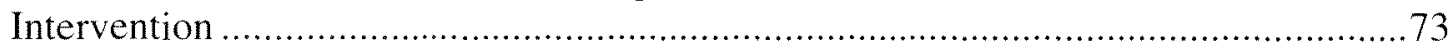

Table 14: Value of Health Outcomes per Non-asthmatic Adult as a Result of the

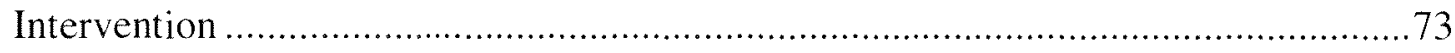

Table 15: Value of Health Outcomes per Household as a Result of Intervention for

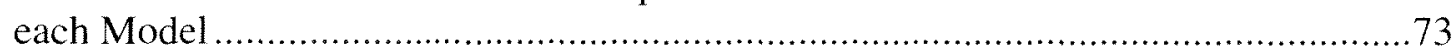

Table 16: Total Energy and Electricity Savings per Household as a Result of

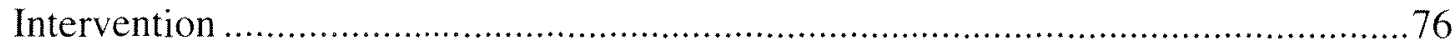

Table 17: The Value of Changes in Comfort per Household as a Result of the

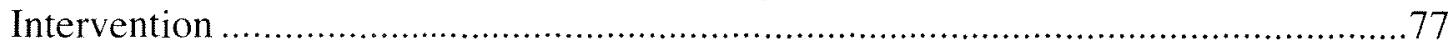

Table 18: Benefit per Household of Reduction in Care-giver Days off Work due to

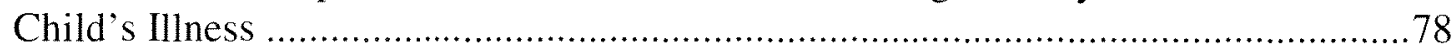

Table 19: NPV of the Intervention per Household over a 10 Year Horizon ................80

Table 20: NPV of the Intervention per Household over a 20 Year Horizon ................81

Table 21: NPV of the Intervention per Household over a 10 Year Period with no

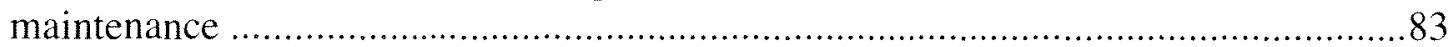

Table 22: NPV of Intervention for Different Heater Types.....................................85

Table 23: NPV of Intervention based on assumption of $10 \%$ and $50 \%$ take-back

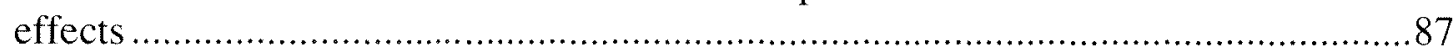

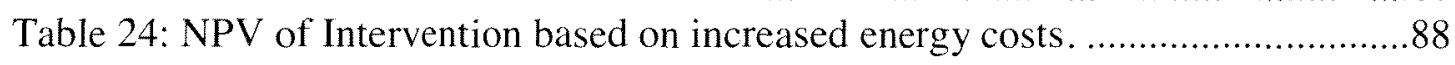




\section{LIST OF ABREVIATIONS}

CCDHD Capital Coast District Health Board

$\mathrm{CO} \quad$ Carbon Monoxide

$\mathrm{CO}_{2} \quad$ Carbon Dioxide

$\mathrm{CO}_{2}-\mathrm{e} \quad$ Carbon Dioxide equivalent

COPD Chronic Obstructive Pulmonary Disease

DBH Department of Building and Housing

EECA Energy Efficiency and Conservation Authority

HNZC Housing New Zealand Corporation

ICD - 10 International Statistical Classification of Diseases and Related Health

Problems, 10th Revision

IPCC Intergovernmental Panel on Climate Change

kWh Kilowatt Hour

LPG Liquefied Petroleum Gas

MED Ministry of Economic Development

MfE Ministry for the Environment

$\mathrm{MOH} \quad$ Ministry of Health

MRR Mean Rate Ratio

MWh Megawatt Hour

$\mathrm{NO}_{2} \quad$ Nitrogen Dioxide

NOx Nitrogen Oxides (including Nitrogen Dioxide)

$\mathrm{PM}_{10} \quad$ Particulate Matter $\leq 10 \mu \mathrm{m}$ in diameter

QALY Quality-Adjusted Life Year

$\mathrm{SO}_{2} \quad$ Sulphur Dioxide

YLD Years Lost to Disability 


\section{INTRODUCTION}

\subsection{The Significance of Domestic Space Heating}

In the global context, the consumption of fossil fuels to keep homes warm and comfortable is an important issue. The Intergovernmental Panel on Climate Change recently stated that: "most of the observed increase in global average temperatures since the mid- $20^{\text {th }}$ century is very likely due to the observed increase in anthropogenic greenhouse gas concentrations" (IPCC, 2007). The impacts of climate change are potentially catastrophic, and the IPCC reports that they may include increased extreme weather events, drought and erosion of coastlines due to rising sea levels (IPCC, 2007). Burning fossil fuels directly or indirectly to heat homes produces greenhouse gases and increases the likelihood of adverse climate change.

New Zealand ratified the Kyoto Protocol and has agreed to reduce its $\mathrm{CO}_{2}$ emissions to 1990 levels during the first commitment period of 2008-2012 or to take responsibility for any excess emissions. Recent projections indicate that New Zealand's greenhouse gas emissions will be around 45.5 million tonnes of $\mathrm{CO}_{2}$ equivalent in excess of its allocated or "target" level during the Kyoto Protocol's first commitment period (MfE, 2007), and it is likely that New Zealand will need to cover its "deficit" by purchasing carbon credits on the international carbon market in order to honour its commitments. Residential energy use produces around $10 \%$ of $\mathrm{New}$ Zealand's $\mathrm{CO}_{2}$ emissions (EECA, 2007) and space heating contributes approximately $34 \%$ of residential energy use (Isaacs et al., 2006). Improvements in domestic space heating efficiency have the potential to reduce emissions and thus have a positive impact on New Zealand's Kyoto deficit and on the physical environment. The 2007 New Zealand Energy Efficiency and Conservation Strategy (NZEECS) estimates that improvements in the energy efficiency of current homes could reduce $\mathrm{CO}_{2}$ emissions by 2.81 million tonnes of per year by 2025 (EECA, 2007).

The burning of fossil fuels for space heating either via electricity generation, or direct consumption of gas or coal has a number of other important negative environmental 
effects. Local emissions from fossil fuel and wood fires include $\mathrm{NO}_{\mathrm{x}}, \mathrm{SO}_{2}$ and $\mathrm{PM}_{10}$, which are all associated with negative health outcomes. New National Environmental Standards for Air Quality enacted under the Resource Management Act 1991 ban the installation of inefficient or excessively polluting woodburners (MfE, 2005); however, it will be many years before inefficient woodburners currently in use reach the end of their life-cycles. Fossil fuel based electricity generation produces similar emissions and is also associated with negative health and ecological impacts. Smog primarily produced by domestic space heating has had a particularly serious impact in Christchurch, due in part to the physical location of the city, and led to the development of Environment Canterbury's Natural Resources Regional Plan which mandates a large reduction in emissions from wood and coal fires (Hales et al., 1999; ECAN, 2007).

Living rooms in New Zealand dwellings have an average evening indoor winter temperature of $17.9^{\circ} \mathrm{C}$ (French et al., 2007), which is slightly below the WHO recommended minimum of $18^{\circ} \mathrm{C}$ (WHO, 1987), and almost $25 \%$ have average winter living room temperatures below $16^{\circ} \mathrm{C}$ (Buckett, 2007). When compared to other OECD nations New Zealand homes are colder, and less well heated (Rankine, 2005). Research suggests that the consequences for occupants of homes with low indoor temperatures and the dampness and mould that they are associated with may include a higher rate of asthma symptoms and other respiratory illnesses, increased mortality and impaired mental health (Hunt, 1990; Howden-Chapman, 2004; HowdenChapman et al., 2007; Mudarri and Fisk, 2007; Wilson et al., 2007).

The WHO assessment "The Global Burden of Asthma" states that in 2003 New Zealand had a population asthma rate of $15.1 \%$, which was approximately three times the world average (Masoli et al., 2004). The reasons why rates of asthma in New Zealand and other English speaking nations such as England, Scotland and Australia are relatively high are not clear but the consequences are. An assessment carried out by Holt and Beasley (2001) for the Asthma and Respiratory Foundation of New Zealand conservatively estimates that asthma cost New Zealand $\$ 800$ million per year during the late $1990 \mathrm{~s}$. 
Low indoor temperatures have been correlated with poor insulation and ineffective space heating. Prior to 1977 , when new regulations required that homes be built with insulation, a large proportion of New Zealand homes were constructed with poor or no insulation, and are therefore difficult to heat (Isaacs et al., 2006; Lloyd, Bishop and Callau, 2007).

Recent housing interventions in New Zealand have demonstrated improvements in indoor temperatures following the installation of basic insulation (Howden-Chapman et al., 2007; Lloyd et al., 2007). The Housing, Insulation and Health Study carried out by He Kainga Oranga, the Housing and Health Research Programme of the School of Medicine and Health Sciences of the University of Otago, Wellington also demonstrated that such improved temperatures lead to significant health benefits for householders (Howden-Chapman, et al., 2007). In addition, improved insulation may lead to a reduction in energy costs and $\mathrm{CO}_{2}$ emissions (Chapman et al. 2007, in review).

Another significant method of improving indoor temperatures is the installation of energy efficient and healthy heaters (Lloyd, Bishop and Callau, 2007). Dwellings heated using unflued gas heaters or portable electric heaters are colder on average than dwellings heated using solid fuel or natural gas (French et al., 2007). New domestic space heating technologies such as heat pumps and wood pellet burners that have become widely available in the last ten years are considerably more effective than older heat sources such as unflued gas heaters and plug-in electric heaters and offer much potential in this area. In addition, these heaters produce low levels of harmful local pollutants such as $\mathrm{NO}_{\mathrm{x}}$ and $\mathrm{SO}_{2}$ relative to their heat output (MfE, 2005).

The potential for energy efficient domestic space heating to positively impact on respiratory health was the basis of the Wellington School of Medicine and Health Sciences' Housing, Heating and Health Study which was carried out by He Kainga Oranga. The Study involved the installation of energy efficient and healthy heaters in the homes of families with an asthmatic child aged seven to twelve, and the data collected demonstrate a number of positive outcomes, including increased living room 
temperatures and reduced visits to health professionals and days off school for asthmatic children, (Howden-Chapman, et al, 2008, accepted with revisions).

While outcomes such as those described above are clearly positive, from a policy making perspective it is important that an assessment of the costs and benefits associated with such an intervention be available to assist with any decision to publicly fund or promote such heaters. An assessment of the Housing, Insulation and Health Study carried out by He Kainga Oranga in 2004 suggests a favourable ratio of benefits to costs, and has provided a valuable decision-making tool for relevant government agencies (Chapman et al., 2007, in review). It is the aim of this thesis to produce a comprehensive cost benefit analysis based on the data produced by the Housing, Heating and Health Study that will be of similar value.

\subsection{Aim}

To carry out a comprehensive cost benefit analysis of space heating improvements based on the data produced by the Housing, Heating and Health Study carried out by He Kainga Oranga.

\subsection{Objectives}

1. Analyse available data from the Housing, Heating and Health Study using appropriate statistical techniques and provide an estimate of the costs and benefits involved;

2. Address issues beyond the direct scope of the data: mortality, mould and structural damage, new cases of asthma avoided, comfort/mental health, embodied costs, caregiver days off work, non $\mathrm{CO}_{2}$ external emissions and the potential of the heat pump to be used as an air conditioner;

3. Construct a model to enable the data outcomes produced as part of objectives One and Two to be projected over a reasonable horizon of analysis; 
4. Draw conclusions as to the overall balance of costs and benefits of installing more effective domestic space heating, taking into account a sensitivity analysis of the outcomes.

\subsection{Structure of Thesis}

Chapter 1 has provided a general overview of the significance of domestic space heating and of the context in which the Housing, Heating and Health study took place, as well as the aims, objectives and scope of this thesis.

Chapter 2 explores in greater detail the potential ways that improved domestic space heating may affect people and the environment. The potential impacts of improved domestic space heating on respiratory, circulatory and mental health are discussed, with a particular focus on asthma. Key research from previous housing interventions is summarised and a full discussion of the structure and published outcomes of the Housing, Heating and Health Study is included. The results of previous economic analyses of housing improvements and the outcomes of previous cost benefit analyses of improvements in home energy efficiency, in particular the Housing, Insulation and Health Study, are discussed.

Chapter 3 focuses on methodology and falls into three main sections. Section 3.1 outlines the conceptual framework of this thesis. Section 3.2 presents the model that has been devised in order to project costs and benefits over the period that the study heaters are predicted to be in operation. Key model components such as heater replacement and maintenance, discount rates, population mobility and the extrapolation of winter data are discussed. Section 3.3 presents the methodology for analysing the costs and benefits that can be directly derived from the Housing, Heating and Health Study data. Section 3.4 explores potential costs and benefits that are beyond the scope of the Study data such as mental health and comfort, summer heat pump air conditioning, cases of asthma avoided, reduced mortality, and the value of reduced damp and mould from a structural perspective.

Chapter 4 presents the results of the cost benefit analysis. The significance of key variables such as the discount rate is explored for each model. 
Chapter 5 discusses the results presented in the previous Chapter and explores the conclusions that can be drawn from them. Potential limitations and flaws of the data, the model and the analysis are discussed. Future research directions are suggested, and the results are placed in the New Zealand housing, energy and health context.

Chapter 6 is a brief summary of the conclusions that can be drawn based on the results of the cost benefit analysis and the discussion.

\subsection{Positionality}

I am a middle class Pakeha male with strong ties to New Zealand. I am in favour of an approach to economics which takes account of environmental externalities. Before carrying out my thesis I was open minded about the potential of the installation of energy efficient domestic space heating to produce positive net economic outcomes.

My thesis supervisor Associate Professor Ralph Chapman is one of the researchers involved with the Study that my analysis is based on; however I do not believe this has affected the objectivity of my analysis. I also worked closely with a number of He Kainga Oranga members in designing and implementing my analysis, but again I do not feel that this involvement has compromised my neutrality as a researcher. 


\section{BACKGROUND}

\subsection{Housing and Heating in New Zealand}

New Zealand's climate is temperate and has a range of average annual temperatures from 16 degrees in the north to 10 degrees in the far south (Howden-Chapman et al. $2007, \mathrm{p} 2$ ). The lower South Island is considerably colder than the upper North Island, with Dunedin having 2580 heating degree days (base of $18^{\circ} \mathrm{C}$ ) in comparison with Auckland which has only 1150 heating degree days (base of $18^{\circ} \mathrm{C}$ ) (Lloyd, Bishop and Callau, 2007). Average yearly temperatures in New Zealand are predicted to rise $0.5-0.7$ degrees by the $2030 \mathrm{~s}$ as a result of global warming, with the majority of the increase occurring in the winter months (NIWA, 2006). This is predicted to reduce heating degree days to a greater extent than it will increase cooling degree days (MED, 2006).

New Zealand's housing stock is of a relatively poor average quality, with average indoor temperatures that are below WHO recommendations (Lloyd et al., 2007). Some authors estimate that one quarter of New Zealand homes currently lack insulation (Rankine, 2005), while others suggest a figure of ten percent (MfE, 2004). As mentioned in Chapter 1, insulation only became mandatory in 1977, after the 1973-74 oil shock, which means that houses built before 1978 are generally colder and damper as they are more difficult to heat economically (Lloyd, Bishop and Callau, 2007). A recent study carried out by BRANZ estimated that homes built after 1978 were $1.0^{\circ} \mathrm{C}$ warmer on average than pre-1978 homes (Isaacs et al., 2006). As homes in New Zealand last on average about 95 years (Isaacs et al., 2005), it is likely that without improvement these homes will continue to have an impact on New Zealand's economy in terms of health and energy use costs for a long time to come.

In 1996 the New Zealand Building Code was revised to require greater consideration of, among other things, energy efficiency in new building design and new hot water systems. The Building Code confirmed and did not largely alter the 1977 insulation standard (Cogan, 1996). The Building Code is currently under review and it is 
possible that higher standards may be imposed on new buildings in relation to the potential for thermal comfort (DBH, 2007).

Recent trends in home ownership patterns include an increase in the proportion of households that are renting (MfE, 2004; Statistics NZ, 2007). Many of the rental properties occupied may be older homes with a lack of insulation and efficient heating (MFE, 2004). This increase is significant because tenants are generally less likely than owner-occupiers to improve their insulation or their space heating arrangement, given that they are unlikely to occupy the home for the life-span of the improvement and that some of the benefits of their investment are likely to be received by the owners of the property or future tenants (MfE, 2004). In turn, landlords may be unlikely to improve insulation or space heating efficiency due to a lack of demand in the rental marketplace (MfE, 2005), although awareness of the benefits of energy efficiency amongst the general public may be increasing, which may in turn increase demand.

EECA is currently in the process of introducing a HERS (Home Energy Ratings Scheme), a voluntary scheme that will enable landlords/sellers to advertise the energy efficiency of a home by obtaining and displaying an efficiency rating (EECA, 2008). Energy efficiency measures captured by HERS will include domestic space heating efficiency. This will potentially make it more economically attractive for landlords/sellers to improve the energy efficiency of their properties as the credibility of the rating increases the likelihood that they may be rewarded with higher rental returns or sale prices. It remains to be seen what impact a voluntary HERS will have on New Zealand's housing market.

Other domestic energy efficiency initiatives include EECA's EnergyWise programme which provides grants to low-income households for basic insulation measures (EECA, 2008A). There are a also number of initiatives at a local government level such as Environment Canterbury's Clean Heat Project which provides 10 year interest free loans to enable households to purchase low emission domestic space heaters including heat pumps, fixed flued gas heaters and pellet burners as part of its efforts to reduce local emissions in accordance with its Natural Resources Regional Plan and the National Environmental Standards for Air Quality (ECAN, 2006). 
Housing New Zealand Corporation (HNZC) is currently undertaking a 10-12 year insulation retrofit programme of its pre 1978 housing stock. The Energy Efficiency Retrofit Programme involves the installation of ceiling, floor and hot-water cylinder insulation and of draught stoppers. It is estimated that by 201245,000 of HNZC's 65,000 homes will have been upgraded (Lloyd et al., 2007).

\subsubsection{Energy and domestic space heating in New Zealand.}

New Zealand homes utilise a variety of space heating methods. According to 2006 Census data, $74.8 \%$ use electricity as a fuel source, $13.2 \%$ use mains gas, $27.7 \%$ use bottled gas, $40.0 \%$ use wood, $7 \%$ use coal, $1.1 \%$ use solar power, $2.1 \%$ use other fuels and $2.4 \%$ do not use any fuel (Statistics NZ, 2007). A MfE report produces similar outcomes based on a nationwide survey, differing significantly only in that it reports a lower proportion of homes (57\%) using electricity as an energy source for space heating (MfE, 2004). The relatively small sample size of the nationwide survey utilised by the MFE suggests that 2006 Census data may be more reliable.

The MfE (2004) commissioned survey indicates that only a small proportion of households use energy efficient healthy heaters of the type installed as part of the Housing, Heating and Health Study: 9\% of households reported a flued gas heater, and $0 \%$ reported a pellet burner, while of the $74.8 \%$ of homes who used electricity as a fuel source, $13 \%$ reported that they owned a heat pump suggesting $9 \%$ of homes possess a heat pump. Buckett (2007) estimates that $7.5 \%$ of households may have a heat pump, based on Clark et al. (2005), and notes the rapid uptake in heat pump use in the last decade. Recent research by French (2008) confirms this trend, with 80,000 heat pumps sold in New Zealand in 2007. The reason for the low uptake of pellet burners is likely to be higher upfront capital costs, combined with a lack of information about the potential financial and health savings available, and personal preferences (PCE, 2006).

Historically New Zealand has had an extremely low space heating intensity measured in $\mathrm{GJ} /$ capita/annum relative to other nations (Lloyd et al. 2007). At first glance this is surprising given that electricity in New Zealand has historically been cheap in 
comparison with other OECD nations (Lloyd et al., 2007); however it is likely to be a natural consequence of poor insulation and fuel poverty. Electricity has been relatively cheap in New Zealand primarily due to the high proportion of renewable electricity available from New Zealand's hydroelectric dams, the negative externalities of which have not been incorporated into electricity prices. Locally extracted gas is used for electricity generation in New Zealand, however New Zealand's major source, the Maui field, is projected to reach the end of it's economic life within ten years, which, despite projected new discoveries, may ultimately entail the importation of gas and is predicted, when combined with the costs associated with building new generation capacity in order to meet demand, to increase the real price of residential electricity by up $20 \%$ (MED, 2006). It is not certain what effect such an increase may have on future domestic space heating related decisions and behaviours at a household level; however, research suggests that the demand for electricity is relatively price inelastic, which implies limited change (Parti and Parti, 1980; EECA, 2003, MED, 2006).

Despite historically relatively inexpensive electricity, recent model based research suggests that $10-14 \%$ of New Zealand households may be in fuel poverty, which is a similar proportion to that of households in England and Scotland, but much lower than Northern Ireland and Wales (Lloyd, 2006; Shortt and Rugkasa, 2007). Fuel poverty is a concept which originated in the United Kingdom in the 1980s and is now in use in many countries including New Zealand, although definitions and interpretations vary (Lloyd, 2006). In Britain a household is said to be experiencing fuel poverty if it would need to spend $10 \%$ or more of its total income on household fuels in order to achieve indoor temperatures of $21^{\circ} \mathrm{C}$ in the living room and $18^{\circ} \mathrm{C}$ in other areas of the home for 16 hours per day, in the case of homes with occupants at home all day, and 9 hours per day for households who work or study full-time (Lloyd, 2006; Shortt and Rugkasa, 2007).

\subsection{Connections between Housing, Heating and Health}


An overview of the available literature highlights four main potential connections between improved domestic space heating and health. These are respiratory health, circulatory health, mental health, and reduced heating costs.

\subsubsection{Respiratory health}

The respiratory tract consists of an upper and lower portion. The upper portion includes the sinus cavities, nose and the throat. Illnesses of the upper respiratory tract potentially affected by improved domestic space heating include allergic rhinitis (Howden-Chapman, 2004; Wilson et al., 2007). Allergic rhinitis is an inflammation of the mucus membrane of the nose resulting in excess mucus and nasal congestion. Allergic rhinitis is caused by airborne allergens, including the domestic space heating related allergens which can also cause symptoms in asthmatics. The connections between asthma and domestic space heating are summarised in the following section.

\subsubsection{Asthma}

Asthma is a chronic respiratory illness that has been extensively studied; however the reasons why people develop asthma are still not fully understood (Masoli, et al, 2004) Asthma involves the constriction and inflammation of the respiratory system in response to a trigger such as an allergen, exercise or cold air. This constriction can lead to coughing, wheezing and breathlessness and in extreme cases unconsciousness and even death from respiratory arrest. Asthmatics typically function well when not experiencing an episode, but may suffer from longer periods of shortness of breath after physical activity than non-asthmatics.

The literature suggests three potential connections between improved space heating and asthma. These are increased temperatures; reduced dampness and mould due to increased temperatures and/or the elimination of water vapour emissions from unflued gas heaters; and a reduction in the local emission of pollutants such as $\mathrm{NO}_{\mathrm{x}}$. 
Temperatures lower than 15 degrees have been correlated with increased severity of asthma episodes (Evans et al., 2000; Howden-Chapman, 2004), which suggests that improving space heating and thus, potentially, average indoor temperatures, will reduce the severity of asthma episodes for sufferers.

Dampness is the result of excess moisture in a building and can result from low temperatures because cold air increases the possibility of condensation (Rankine, 2005). Water vapour is a product of combustion, and if it is released internally (as is the case with unflued gas heaters), it will also potentially cause increased dampness (Howden-Chapman, 2004). Dampness is related to asthma symptoms in two ways. Firstly, humid environments are more favourable to dust mite survival and population size. The faecal allergens (e.g. Der $\mathrm{p} \mathrm{I}$ and Der $\mathrm{f}$ I) that dust mites produce are one of the main sources of indoor allergens which can affect sensitised asthmatics (Carrer et al., 2001). Secondly, dampness increases the possibility of mould growth, and inhaling moulds and other microbial allergens can produce allergic reactions in sensitised asthmatics as well as causing non immune specific inflammation which asthmatics may be more susceptible to (Carrer et al. 2001, Howden-Chapman, 2004).

There have been a number of literature reviews analysing the connection between dampness and asthma. Fisk, Gomez and Mendell (2007) carried out a meta-analysis of a review by the Institute of Medicine of the U.S National Academy of Sciences of the connections between dampness and health. Although they faced a number of difficulties, including impreciseness and variability in definitions of damp and mould and of asthma symptoms, they were able to analyse 33 studies and established statistically significant odds ratios for a variety of measures including upper respiratory tract symptoms, wheeze, current asthma, and asthma development of between 1.34 and 1.70 , generally with a lower bound of 1.20 or greater (p 287). An earlier review by Peat, Dickerson and Li (1998) produced similar results, although their outcomes exhibited greater variability.

$\mathrm{NO}_{\mathrm{x}}, \mathrm{SO}_{2}$ and $\mathrm{PM}_{10}$ are products of combustion which can irritate airways. Unflued gas heaters release $\mathrm{NO}_{x}$ into the indoor environment. A number of studies have reported a relationship between indoor $\mathrm{NO}_{2}$ levels and asthma symptoms (Chauhan et al., 2003, Melia, et al., 1982; Pilotto, 2003). One explanation of the connection 
between $\mathrm{NO}_{2}$ levels and asthma symptoms is that $\mathrm{NO}_{2}$ can increase the effect of allergens such as dust-mites on sensitised asthmatics (Tunnicliffe, 1994, Barck et al., 2002).

\subsubsection{COPD}

Chronic obstructive pulmonary disease (COPD) is an obstruction of the airways which can be caused by chronic bronchitis or emphysema. COPD is generally experienced by middle aged and older people who have been exposed to cigarette smoke (Kerstjens and Postma, 2002). COPD is a leading cause of mortality and morbidity in New Zealand, resulting in 30,115 YLD each year in the late 1990s (Holt and Beasley, 2002). A seasonal variability in hospital admissions for COPD has been observed, as low winter temperatures can lead to bronchoconstriction in patients and reduce the effectiveness of the thermoregulatory system in older patients (Marno, 2006). Older people typically spend a greater than average amount of time at home (Howden-Chapman, 2004) and improved indoor temperatures due to more efficient domestic space heating could potentially reduce acute symptoms and discomfort for COPD sufferers.

In addition, an $8.9 \%(3.0: 15.2,95 \% \mathrm{CI})$ increase in COPD related mortality per 10 $\mu \mathrm{gm}^{-3}$ increase in atmospheric $\mathrm{NO}_{2}$ levels, in the 14 days following the increase, and a $5.1 \%(1.3: 9.1,95 \% \mathrm{CI})$ increase per $10 \mu \mathrm{gm}^{-3} \mathrm{PM}_{10}$ was recorded in a recent study, suggesting that emissions from domestic space heating could potentially have similar negative impacts on COPD sufferers (Neuberger, Rabczenko and Moshammer, 2007).

\subsubsection{Influenza}

Influenza is an infectious viral disease. In humans, it can cause a variety of symptoms including fever, sore throat, pain, coughing and exhaustion. Influenza can also cause pneumonia, particularly in young children and older people; this is more serious and can lead to death. 
Influenza exhibits seasonal variation, with winter peaks in morbidity. Research suggests a number of potential explanations for this variability including suppression of resistance to respiratory infection due to cold stress and increased opportunity for infection to spread due to greater time spent indoors in close confines (Keatinge et al., 1997). A review of excess winter mortality in Europe found a statistically significant correlation between living room temperature and death from all respiratory diseases (including pneumonia) (Keatinge et al., 1997); however a causal connection between indoor temperature and influenza prevalence is not generally accepted.

\subsubsection{Circulatory health}

A review of excess winter mortality in New Zealand reported a ratio of winter to nonwinter mortality for ICD-10 defined circulatory disease of 1.2:1 (1.15 - 1.24; 95\% CI) (Davie et al., 2007). The ICD-10 classification for circulatory disease includes ischaemic heart disease (heart attacks) and cerebrovascular disease (strokes) which were the two leading causes of disability adjusted life years lost in New Zealand in 1996 ( Holt and Beasley, 2001).

\subsubsection{Ischaemic heart disease}

Ischaemic heart disease is the end result of the accumulation of atheromatous plaques within the walls of the arteries that provide the muscle of the heart with oxygen and nutrients. Many individuals with coronary heart disease demonstrate no symptoms for decades while the disease progresses; the first symptoms noticed may be a fatal heart attack.

Ischaemic heart disease has been related (but not significantly) to low indoor temperatures by some research (Keatinge et al., 1997). The causal mechanism proposed by some researchers is increased systolic and dystolic blood pressure caused by exposure to cold temperatures which causes increased oxygen consumption by the heart (Aylin et al., 2001; Pelle and Cobbe, 1999). Evidence that suggests a correlation between lower indoor temperatures and circulatory disease includes a pattern of 
variation in excess winter mortality in Europe which correlates the quality of building insulation and the availability of cheap energy with ischaemic heart disease mortality (Pell and Cobbe, 1999). However there is evidence which makes the connection less clear such as the fact that people at higher altitudes (which are colder) have lower rates of ischaemic heart disease (Fabsitz and Feinlieb, 1980; Pell and Cobbe, 1999). A number of other potential explanations include the theory that exposure to sunlight (and production of vitamin D) may protect people from ischaemic heart disease, and thus reduced hours of sunlight could explain excess winter mortality (Pell and Cobbe, 1999). Exposure to cold outside the home, higher rates of respiratory infection, inactivity, increased winter obesity, increased winter cholesterol levels and changes in blood coagulation factors may all contribute to excess winter mortality (Pell and Cobbe, 1999).

\subsubsection{Cerebrovascular disease}

Cerebrovascular disease is a collective term for a group of diseases, including strokes, which are characterized by defects of the arteries of the brain, or of the arteries connected to the brain.

Cerebrovascular disease has been correlated (not significantly) with low indoor temperatures by some research (Keatinge et al., 1997). A literature review uncovered relatively little research attempting to explain excess winter mortality from cerebrovascular disease. One potential mechanism identified is arterial thrombosis caused by increased haemoconcentration resulting from exposure to cold temperatures (Keatinge et al., 1997).

In general, the correlation between circulatory disease and housing, heating and health is less strong than the correlation between respiratory disease and housing, heating and health (Mudarri and Fisk, 2007).

\subsubsection{Other potential health effects}


Rheumatism is a non technical term which encompasses a wide variety of conditions including arthritis. Some research has found a statistically significant correlation between improved household energy efficiency and a decreased prevalence of self reported arthritis/rheumatism (Iverson, Bach and Lundqvist, 1986; Shortt and Rugkasa, 2007). Other studies have not reported a statistically significant change in self reported arthritis or rheumatism following a heating intervention (Somerville et al., 2002), and the IOM (2004) concluded that there was insufficient or inadequate evidence of a connection between damp or mouldy homes and rheumatologic and other immune diseases.

\subsubsection{Mental health}

Poor mental health is a broad category which includes both negative affect and psychiatric disorders. There is a reasonably strong link between housing and mental health (Howden-Chapman, 2004); however, as with other aspects of health and housing, the connection has been under researched (Evan, Wells and Moch, 2003).

A fundamental difficulty, regarding the connection between housing and mental health, is to establish to what extent poor housing is causative of, rather than correlated with, poor mental health (via, for example, poverty or inability to maintain home appropriately). An additional difficulty is that research has often used subjective reporting of both home condition and of mental health, making it difficult to assess to what extent self-reporting of the housing problem in question is coloured by poor mental health (Evan, Wells and Moch, 2003, p 490).

The author was not able to identify any research that focused exclusively on the potential connection between domestic space heating and mental health, but a number of studies have explored the connection between poor housing quality (including dampness and structural flaws) and mental health.

Hopton and Hunt (1996) found a correlation between dampness (measured via assessment of 6 dampness related problems) and poorer mental health in 451 Scottish homes when controlling for socio-economic status. Hunt (1990), found a correlation 
between the number of housing problems (including damp and cold) assessed by surveyors, and the proportion of adult and child occupants who reported psychological distress (Evan, Wells and Moch, 2003).

This, and other similar research (Gilbertson et al., 2006), indicates that there is likely to be an improvement in mental health as a result of improved domestic space heating, given the various correlations between cold, damp and domestic space heating. The potential mechanisms may include improved self-esteem and self perception (Evan, Wells and Moch, 2003), reduced stress from perceived failure to meet normative standards (Harrington et al., 2005), reduced financial stress (Harrington et al., 2005), improved physical comfort, reduced stress and improved relationships resulting from increased usable (warm) space in homes (Gilbertson, et al. 2006), reduced emotional stress resulting from ill health, and reduced anxiety about ones own health or the health of other family members, particularly in the case of parents with asthmatic children.

People's interaction with their homes is complex, however, and previous experience and expectations regarding domestic space heating are likely to influence, mitigate or even exacerbate the psychological impact of poor space heating, which will in turn influence the potential benefit of improved space heating (Harrington et al., 2005).

\subsubsection{Heating costs and health}

Reduced heating costs will potentially have the greatest impact on households currently experiencing fuel poverty or that include members who may be in poor health or particularly susceptible to the effects of low temperatures, dampness and mould.

On a practical level, improvements in domestic space heating efficiency can potentially reduce costs, thus freeing money to be spent on other essentials such as food or medication which have the potential to improve occupant health (HowdenChapman et al, 2007). 


\subsection{Housing Interventions and Health}

Epidemiological studies have demonstrated strong links between housing and health outcomes; however, epidemiological studies cannot demonstrate causal links in the way that interventions can (Thomson, Petticrew and Morrison, 2002).

Thomson, Petticrew and Morrison (2002) carry out a comprehensive literature review of housing interventions and health which concluded that there was a surprisingly small quantity of evidence of a connection between improved housing and health. The authors were able to identify only 19 relevant intervention studies, 9 of which included a control group, and point out the methodological difficulties inherent in housing intervention studies such as the complex interactions of multiple factors in producing outcomes and the fact that is difficult/impossible to blind occupants to changes in their environment. Despite difficulties in comparing data from different interventions the authors conclude that housing interventions generally have a positive but inconsistent impact on self reported physical and mental health.

Since 2002 the outcomes of several new housing intervention studies have been published. The Watcombe Housing Study, carried out between 1999 and 2001, assessed the effects of installing an upgrade package including wet central heating, wall and loft insulation, double glazing on doors and on-demand ventilation into single family unit social housing in an area with a high deprivation index (Richardson et al., 2006). Participants were randomised into two groups (Phase I and II) in 1999 and a variety of health and environmental data were recorded for all homes. Phase I homes had the upgrade package installed prior to data collection in early 2000 and Phase II homes were upgraded prior to data collection in 2001. There were few significant differences in health outcomes between Phases when comparing 1999 and 2000 data, other than with regard to non-asthmatic respiratory illness and adult asthma symptoms. During the 3 year period of analysis asthma prevalence was reduced in children but this reduction was not linked to the upgrade package, according to unpublished research by Barton et al. (Richardson et al., 2006, p 77)

Kercsmar et al. (2006) target mouldy homes occupied by asthmatic children. They provided a variety of housing upgrades including improved heating for intervention 
homes, but health advice only for control group homes. Positive outcomes include a statistically significant decrease in both symptom days and in the exacerbation of symptoms in the intervention group only.

Walker et al. (2006) study the Scottish Central Heating Programme which provides central heating and ceiling and pipe insulation and assessed the impact of retrofits using a control group of non-recipients. The authors find a strong correlation between three health outcomes including wheezing, and mould, cold and condensation. The three health outcomes were in turn correlated with levels of heating use, although paradoxically, self reported health was not associated with levels of heating use.

Shortt and Rugkasa (2007) compare pre and post-intervention self reported health data, for 54 homes that were part of a fuel poverty programme in Northern Ireland involving the installation of energy efficiency measures including improved central heating. They report statistically significant reductions in the post-intervention prevalence of arthritis/rheumatism, other illnesses and mean number of illnesses per person, but not, as might have been predicted, of respiratory illnesses ( $\mathrm{p} 106$ ).

In general, until recently, there has been a lack of research published on the health impacts of housing interventions, and insofar as the results are comparable, they are inconsistent and inconclusive. Whether the lack of research is due to the self evident nature of the connection between housing and health, or the difficulties outlined earlier, is difficult to ascertain. In this context the research produced by the Wellington School of Medicine and Health Sciences is particularly valuable.

The Housing, Insulation and Health Study was a single blind randomised study which involved the installation of a standard retrofit insulation package into uninsulated homes with at least one member who had experienced wheezing or other respiratory symptoms in the previous year. 1350 households from seven locations in New Zealand were randomised into intervention and control groups and baseline data including SF-36 self report scales, days off work/school, GP visits and hospitalisations were recorded following the winter of 2001. In 2002, prior to the winter, the retrofit was carried out in intervention group homes and at the end of the 
winter the same data set was collected. After the second winter the retrofit package was installed in the control group homes (Howden-Chapman et al., 2007).

Comparison of data from the two groups, primarily using ANCOVA, demonstrated statistically significant favourable adjusted odds ratios for self reported mould, cold, condensation, non-condensation related dampness, ineffective heating and combined self reported and measured energy use. Data recorded for a sub-sample of homes demonstrated a statistically significant increase in temperature of $0.5^{\circ} \mathrm{C}$, and a reduction in relative humidity, in the average number of hours per day colder than $10^{\circ} \mathrm{C}$, and in average hours/day with more than $75 \%$ humidity.

Health outcomes included statistically significant reductions in the adjusted odds ratios for SF-36 self report scale responses for low vitality, low happiness and fair or poor general health. Self reported respiratory symptoms such as wheezing, colds and morning phlegm were also reduced. Days off work for working adults and days off school demonstrated a statistically significant reduction as did self reported GP visits. There was no statistically significant reduction in GP reported visits, which could potentially be explained by the fact that people may use multiple GPs, or in admissions to hospital for respiratory conditions (Howden-Chapman, et al., 2007).

\subsubsection{The Housing, Heating and Health Study}

Following the Housing, Insulation and Health Study, the Housing, Heating and Health Study was a similar randomised community trial which was carried out between 2005 and 2006. It involved the installation of heat pumps, flued gas heaters and pellets burners into households with an asthmatic child, and recording the impact of this intervention on occupant health outcomes and energy usage.

The key characteristics necessary for a household to be included in the study were; having a child aged seven to twelve with doctor diagnosed asthma who had used asthma medication in the last twelve months and had asthmatic symptoms (the index child), and currently using either an unflued gas heater or a plug-in electric heater as the main form of heating (Howden-Chapman et al., 2005). 
Households in five locations - Hutt City and Porirua at the base of the North Island and Christchurch, Dunedin and Bluff in the South Island were recruited by researcher trained community co-ordinators who were identified by local primary health organisations who had been previously contacted. The community coordinators worked with households to ensure that data was collected accurately and in a timely fashion.

Prior to the winter of 2005 , households that had inadequate insulation were insulated in accordance with the minimum standards set out in the New Zealand Building Code in order to eliminate this potentially very important confounding variable (HowdenChapman et al., 2008, accepted with revisions). Households met with researchers to discuss the advantages and disadvantages of the heating options that were offered to them. Owner-occupiers selected a heater, while in the case of tenants the decision was made by their landlord.

A variety of data were collected during and after the winter of 2005 including seven questionnaires/forms, temperatures which were collected using a Thermocron ibutton, $\mathrm{NO}_{2}$ levels recorded using Palmes tubes and the lung function of index children recorded via PIKO meters. Forms completed by households included health questionnaires for each member of the family, daily health diaries for the index children, energy use questionnaires and a Head of Household questionnaire which covered a wide a variety of information including energy use, perceptions of coldness and dampness in the home, space heating choices and relevant household characteristics. Intensive monitoring of a subset of 40 households in Hutt City also took place involving the collection of measures such as $\mathrm{NO}_{2}$, fungi, moisture, carbon monoxide, carbon dioxide and formaldehyde. In addition, independently recorded data such as school attendance records, GP appointment records and power bills were collected (Howden-Chapman et al., 2008, accepted with revisions).

Of the 521 households who were accepted for baseline measures, 459 were still enrolled at the end of 2005 . Of that 459 a further 50 households were lost for a variety of reasons including having moved house, no forms being received and no heater choice having been made (Howden-Chapman et al., 2005; Howden-Chapman et al. 
2008, accepted with revisions). The remaining 409 households were randomised by an independent biostatistician into a control group of 209 and an intervention group of 200. The control group and intervention groups were similar with regard to all key characteristics (Howden-Chapman et al., 2008, accepted with revisions, p 9).

The new heaters were installed in intervention group homes prior to the winter of 2006. The same questionnaires and other measures filled out during and after the 2005 winter were filled out after and during the 2006 winter, with some minor modifications. Questionnaire design was altered to take account of the fact that some questions had been poorly answered in the previous year and that new questions were appropriate for those homes that had new study heaters. Of the 209 control and 200 intervention group households, 174 and 175 were still part of the study at the end of the study respectively; 60 households having withdrawn or been withdrawn for a variety of reasons.

After the study was completed the heaters chosen by the control group households were installed in early 2007.

\subsubsection{Data from the Housing, Heating and Health Study}

Data from the study were double entered and cleaned, and then analysed using $R$ project software R version 2.4.1 (Howden-Chapman, et al., 2008, accepted with revisions, p 8). Health outcome data were analysed using "generalised linear models with the logistic and Poisson log link" (p 9) and daily diary records such as medication use were analysed using generalised linear mixed effects models ( $\mathrm{p} 8$ ). Health data were adjusted for sex, age, ethnicity, parental income, region, smoking in the home and $\mathrm{NO}_{2}$ levels in the child's bedroom and baseline measures if available ( $\mathrm{p}$ 2). Daily records of asthma symptoms and medication use were analysed using generalized linear mixed effects models.

Key initial outcomes included a statistically significant increase in average intervention group living room temperatures of $1.1^{\circ} \mathrm{C}$ and in index children's 
bedrooms of $0.53^{\circ} \mathrm{C}$ and reduced exposure to temperatures below $10^{\circ} \mathrm{C}$ in both rooms. Levels of indoor $\mathrm{NO}_{2}$ were also halved.

Health outcomes reported for intervention group index children include a statistically significant reduction in poor health, dry cough, morning reliever use, numbers of colds/flus, and sleep disturbance from wheeze. Days off school in the winter term were reduced by 1.8 days according to school records, although there was not a statistically significant reduction in parentally reported days off school. Statistically significant reductions the number of in parentally reported GP visits for both asthma and non asthma related problems and asthma related visits to pharmacists were also reported.

The methodology used to analyse the Housing, Heating and Health Study is described further in Chapter 3, following the subsequent section's description of how housing, energy efficiency and health literature has approached economic analysis.

\subsection{Economic Analysis of Housing, Heating and Health}

Economic analyses of improvements in home energy efficiency generally focus on either the value of energy savings or of health improvements. Relatively few studies have comprehensively analysed the combined value of these factors. It is also important to note that none of the studies described below focused exclusively on domestic space heating improvements.

\subsubsection{Energy savings}

Clinch and Healy (2001) review a number of studies which evaluate the financial savings that result from improved home energy efficiency on a household level in terms of reduced energy use, and find that these improvements produce positive net outcomes. They also discuss several macro level studies that indicate similar results for retrofitting homes. However, the authors conclude that these studies are limited by the fact that they focused only on energy savings and in some cases $\mathrm{CO}_{2}$ emissions 
but not on the potential health impacts of the improvements, in some cases because of the difficulties in quantifying these impacts (Clinch and Healy, 2001).

An additional difficulty with some of the studies reviewed was the fact that they did not address the complex interaction between occupant behaviour, occupant economic situation and energy use (Clinch and Healy, 2001).

It is natural to assume that improvements in energy efficiency will result in energy savings. However, this is not necessarily the case due to the take-back effect. The take-back effect refers to any additional consumption that occurs as a result of financial savings due to improved efficiency (Binswanger, 2001; Herring, 2006; Herring and Roy, 2002; Sanne, 2000). In the case of improved space heating, the takeback effect means that households choose to be warmer than they were previously, which is likely to have economic benefits in terms of improved physical and mental health (via the resulting reductions in healthcare costs and increased productivity), but will reduce energy savings. Milne and Boardman (2000) find that in households with an average indoor temperature of $16.5^{\circ} \mathrm{C}$ typically $30 \%$ of energy saved via improvements in household energy efficiency will be "spent" in this manner; the authors find that it is only when a $19^{\circ} \mathrm{C}$ threshold is reached that the take-back effect is reduced to $20 \%$. A similar conclusion regarding the effect of improved energy efficiency on the behaviour of disadvantaged households in Britain is echoed by Gilbertson et al. (2006).

In addition to the take-back effect, there is a complex behavioural element which mediates the impact of improved home energy efficiency. Critchley et al. (2007) analyse qualitative and quantitative data from the England's Warm Front Project, which provides grant funded packages of insulation and domestic space heating improvements for households in fuel poverty, focusing on households who did not demonstrate an increase in indoor temperature following intervention. Of the 888 households analysed (a subset of a larger study), 222 households had remained cold, with mean living room or bedroom temperatures below WHO standards. For $40 \%$ of these cold households coldness was positively correlated with physical qualities of the home such as drafts and with the age of the home. Interviews with 79 of the households revealed that many older people had had trouble using their new space 
heating, which also corresponds with a "rational" explanation of coldness (p 5). However, $40 \%$ of households interviewed reported a preference for colder temperatures, which had not been altered by the intervention as predicted by theories of thermal creep over time. Harrington et al. (2005) also analyse qualitative interview data from the Warm Homes Project and conclude that participants' responses are consistent with the hypothesis that people do not interact mechanically with their environment and thus that a causal account of fuel poverty and its impact on health and energy use is of limited value ( $\mathrm{p}$ 264). Attitudes, priorities and preferences all have an impact on the space heating decisions and the behaviour of households and cannot be discounted.

Reductions in energy use have been reported by a number of recent studies. Somerville et al. (2002) report that, as a result of the Watcombe Housing Study intervention, SAP scores (the United Kingdom's Standard Assessment Procedure for rating home energy efficiency) increased by an average of 33 points which, following SAP assessment procedures, is predicted to produce a reduction in energy costs of $£ 250$ and a corresponding reduction in $\mathrm{CO}_{2}$ emissions of 2 tonnes per home. However, changes in SAP scores do not take into account factors such as occupant behaviour (DEFRA, 2005), and these results must be viewed in this light.

Shortt and Rugkasa (2007) report a statistically significant reduction in self reported total fuel costs from an average of $£ 1,113(\mathrm{NZ} \$ 2849)$ per annum to $£ 751.56(\mathrm{NZ}$ $\$ 1,924)$ for 54 homes following the installation of energy efficiency measures including central heating.

Other recent research has more ambiguous outcomes, including some New Zealand research. Oreszczyn et al. (2006) analyse data collected for the winters of 2001/2002 and 2002/2003 from 3099 Warm Front scheme homes in five urban locations in England. These homes represented a cross section of pre and post intervention homes and included 390 that were retrofitted between winters, allowing for a longitudinal comparison. The authors conclude that, after standardising average temperature data to take into account external temperatures, homes which received the full insulation and heating intervention had an increase in daytime living room temperatures of $1.6^{\circ} \mathrm{C}$ and of night-time bedroom temperature of $2.8^{\circ} \mathrm{C}$. Hong et al. (2006) review changes 
in metered energy use resulting from the retrofit. Longitudinal comparison of the data finds a $35 \%$ increase in total mean energy consumption, while cross-sectional comparison finds a $15 \%$ increase. The authors had predicted that, based on their model, the Warm Front measures would result in a $25-35 \%$ reduction in normalised space heating energy consumption (Wh/K/m $/ 2$ day); however analysis of cross sectional and longitudinal data did not reveal any statistically significant change in normalised space heating fuel consumption ( $\mathrm{p}$ 1176). The lack of change could not be attributed to increased comfort/temperature, and the authors conclude that the disparity between modelled and actual results either reflected flaws in the assumptions of their model or factors such as poor installation of insulation, unmetered fuel usage changes that were not included in the data or changes in occupant behaviour such as increased opening of windows (p 1178-1180).

Guler et al. (2005), cited by Lloyd et al. (2007), conclude that an insulation retrofit of Canadian homes would have a limited potential for energy savings $(0-8 \%$ of total energy consumption), and would be unlikely to have a payback period of less than twenty years.

New Zealand based research on home energy efficiency has produced outcomes that are reasonably consistent with the international research cited above. Early work by the New Zealand Department of Statistics reviewed temperature and electricity usage for 200 homes, and concluded that while homes with ceiling insulation had $0.5^{\circ} \mathrm{C}$ higher temperatures than non insulated homes for both living rooms and bedrooms, there was no significant difference in the electricity consumed. This result was not consistent with a predicted $30-35 \%$ decrease in electricity use for insulated homes (New Zealand Department of Statistics, 1976).

A review of improvements made by HNZC as part of its New Zealand National Energy Efficiency Retrofit Programme was recently carried out (Lloyd et al., 2007). Temperature and energy use data was recorded for 100 Dunedin homes over a two year period after dividing the homes into two groups, one group having received the upgrade package and the other yet to have it installed. This allowed a comparison between uninsulated and insulated homes to be made during the same winter, and also for the effect of installing insulation between winters to be analysed. The authors 
conclude that the upgrade resulted in a small increase in the annual indoor temperature of both living rooms and bedrooms of $0.4^{\circ} \mathrm{C} \pm 0.2^{\circ} \mathrm{C}$, which is consistent with the Housing, Insulation and Health Study outcomes. When energy use is analysed the authors conclude that there was a statistically significant reduction in electricity use of $10 \%( \pm 3 \%)$ as a result of the intervention based on meter and power company data, but that no statistically significant change in total energy use had occurred due to the large degree of error in self reported "other fuel" data.

More recently a highly technical cost benefit analysis based on improvements to two HNZC homes in Dunedin was carried out by some of the same researchers (Lloyd, Bishop and Callau, 2007). This was the second report of a 5 year project looking at the HNZC energy efficiency retrofit programme, and explored energy efficiency upgrades for HNZC houses beyond the standard upgrade package discussed above, which according to the authors may ultimately prove necessary given the limited improvements reported. The cost benefit analysis uses an uninsulated home with an open coal fire as the base case and assesses a variety of energy efficiency improvements in order to establish the most effective sequence of improvements. The model the authors use assumes no change in indoor temperature (no take-back effect) as a result of upgrades, and focuses entirely on reductions in energy costs. The following optimal upgrade path was established, with all options paying for themselves within a 10 year period:
1) Insulate the ceiling
2) Insulate the floor
3) Install a low emissions wood burner or pellet fire
4) Install a heat pump to replace electric heaters used elsewhere in the house
5) Improve air-tightness
6) Insulate walls
7) Install double glazing (or drapes)

(Lloyd, Bishop and Callau, 2007, p47)

The authors' results are reasonably consistent with those of Verbeeck and Hens (2005) who carried out an analysis of the Belgian residential sector. 
In general, the available research on the effect of home energy efficiency measures such as insulation and improved space heating on energy consumption is difficult to compare and inconclusive, with some studies reporting statistically significant reductions in energy consumption, while other studies report increases in temperature with no statistically significant change in energy usage. It is likely that the impact of an intervention will be strongly influenced by prior conditions; with greater energy savings in dwellings that were already fully heated and greater comfort gains in dwellings that were previously cold (Lloyd, Bishop and Callau, 2007). The complexities of occupant behaviour and the differences between modelled and actual outcomes discussed by Hong et al. (2006) confirm the value of empirical data.

\subsubsection{Health savings}

The author was unable to identify a great deal of research costing the health benefits of improved energy efficiency in housing. Three comprehensive cost benefit analyses that do so are discussed in Section 2.4.3. Research on the health costs of dampness and mould, which can potentially be avoided by improved home energy efficiency, is discussed below.

Mudarri and Fisk (2007) assessed the asthma related health costs of domestic dampness and mould in the United States. They estimated that, based on an odds ratio of 1.56 for current asthma cases in damp/mouldy homes, $21 \%$ of the United States' 21.8 million current cases of asthma could be attributed to dampness and mould in the home. The economic cost in terms of morbidity, mortality and lost work and school days was estimated to be $\$$ U.S. 3.5 billion per year.

Holt and Beasley (2001) carried out a comprehensive assessment of the cost of asthma to New Zealand in the late 1990s. Costing pharmaceutical expenditure, medical care, excess mortality, years lost to disability, days off school and days off work they estimated that asthma cost New Zealand roughly $\$ 825$ million per year in the late 1990s. Following Mudarri and Fisk (2007) above, if $21 \%$ of New Zealand's asthma cases can be attributed to dampness and mould then it is possible to 
speculatively assign a yearly cost of approximately $\$ 173$ million to asthma caused by dampness and mould.

Chapman (2007) carried out a comprehensive analysis of the health costs of dampness and mould resulting from leaky building syndrome ${ }^{!}$. The authors used an independent estimate of the number of damp and mouldy homes that could be attributed to leaky buildings $(30,000)$. Utilising data from Holt and Beasley (2001), the New Zealand Health Information Service and Statistics New Zealand, a conservative estimate of asthma costs per person was made based on hospitalisation costs and outpatient treatment costs of $\$ 866,000$ per year (2006 dollars). The value of increased days off work and school were calculated following Chapman et al. (2007, in review) by assuming that the negative effect of dampness is likely to be similar to the positive effect of insulation.

The author also assessed the potential mental health related costs of dampness and mould. Based on a standard formula for calculating the incremental incidence of a condition, and using an odds ratio of $1.4: 1$ for mental health conditions in damp/mouldy homes (Weich and Lewis, 1998), the author estimated that, given a cost per episode of mental illness of $\$ 6,171$ (following (Layard et al., 2006)) and a cost of suicide of $\$ 448,000$ (O'Dea and Tucker, 2005), leaky building related dampness/mould annually cost New Zealand \$59 million via an increased incidence of mental illness. Over a 10 year period, health related costs from leaky building related dampness and mould were estimated to have a present value, at a $5 \%$ discount rate, of around $\$ 474$ million.

\subsubsection{Comprehensive cost benefit analyses of housing improvements}

There has only been a limited amount of research based on the health or energy use impact of housing interventions and there have been fewer studies which assess the full social implications of such interventions. However, a number of model based

\footnotetext{
'Leaky building syndrome is a phenomenon in which recently built New Zealand homes have suffered structural damage from dampness and mould (extreme in some cases) as a result of inadequate design or construction materials (Consumer Build, 2008).
} 
studies have been carried out in which researchers analyse the costs and benefits associated with improved home energy efficiency.

Clinch and Healy (2001) carry out a comprehensive model based assessment of the effects of improving Ireland's housing stock by installing insulation, draught sealing, double glazing and central heating over a ten year period to meet current building standards. Their results include reduced excess winter mortality and morbidity, and improved comfort, and incorporated the take-back effect. Their comprehensive assessment includes an analysis of the savings in health costs resulting from reduced $\mathrm{SO}_{2}, \mathrm{NO}_{x}, \mathrm{PM}_{10}$ and $\mathrm{CO}$ emissions and incorporated improved comfort. They calculate that, under the most likely scenario, for the thirty year period of analysis, using a 5\% discount rate, the intervention would produce a net social benefit of $€ 3124$ million, with a payback period of seven years and a high internal rate of return of $33 \%$ (Clinch and Healy, 2001, p 121). Energy savings make up 57\% of the benefits, health benefits $25 \%$, comfort benefits $10 \%$, and emissions reductions comprise the remaining $8 \%$.

Goodacre, Sharples and Smith (2002) carry out a similar model based analysis of the English housing stock, assessing the costs and benefits of improving home energy efficiency. They cost reductions in energy use and $\mathrm{CO}_{2}$ emissions, improvements in thermal comfort, employment gains and health saving from predicted reductions in dampness and cold and conclude that, with a $5 \%$ discount rate and a 15 year period of analysis, there would be a net social benefit of $\$ 3165$ million, and a benefit-cost ratio of 1.24:1. Based on an assessment of the $5 \%$ discount case, energy savings make up $38 \%$ of benefits, NHS savings $35 \%$, comfort $16 \%$, employment related savings $9 \%$ and $\mathrm{CO}_{2}$ savings $2 \%$.

The results of research by Goodacre, Sharples and Smith (2002) and Clinch and Healy (2001) are valuable from a policy perspective; however the limitations of model based studies demonstrated, for example, by the difference between predicted and actual energy savings for the Warm Front Project discussed above, suggest that cost benefit analyses based on empirical data are of particular value. In addition, neither study is based on New Zealand data, which limits their value from a New Zealand perspective. The only comprehensive cost benefit analysis of improved home energy efficiency 
based on empirical data that a review of the available literature revealed was the cost benefit analysis of the Housing, Insulation and Health Study; it is thus useful to discuss the results of that analysis separately.

\subsubsection{Cost benefit analysis of the Housing, Insulation and Health Study}

The cost benefit analysis of the Housing, Insulation and Health Study produced a highly favourable ratio of benefits to costs of almost 2:1 (Chapman et al., 2007, in review).

Energy and $\mathrm{CO}_{2}$ savings, health costs, days off school and days off work were all valued. In order to account for the changes in the control and intervention groups relative to the baseline year an ANCOVA based analysis was used; if, for example, the control group increased its energy use by $5 \%$ while the intervention group reduced its energy usage by $5 \%$, the impact of the intervention was considered to be a $10 \%$ decrease in energy use. The benefit of this approach is that it compares changes within the intervention and control groups, thus potentially controlling for variables such as income, family size etc. Table 1 details net benefits and costs over the 30 year period of analysis:

Table 1: Housing, Insulation and Health Study: Costs and Benefits per Household

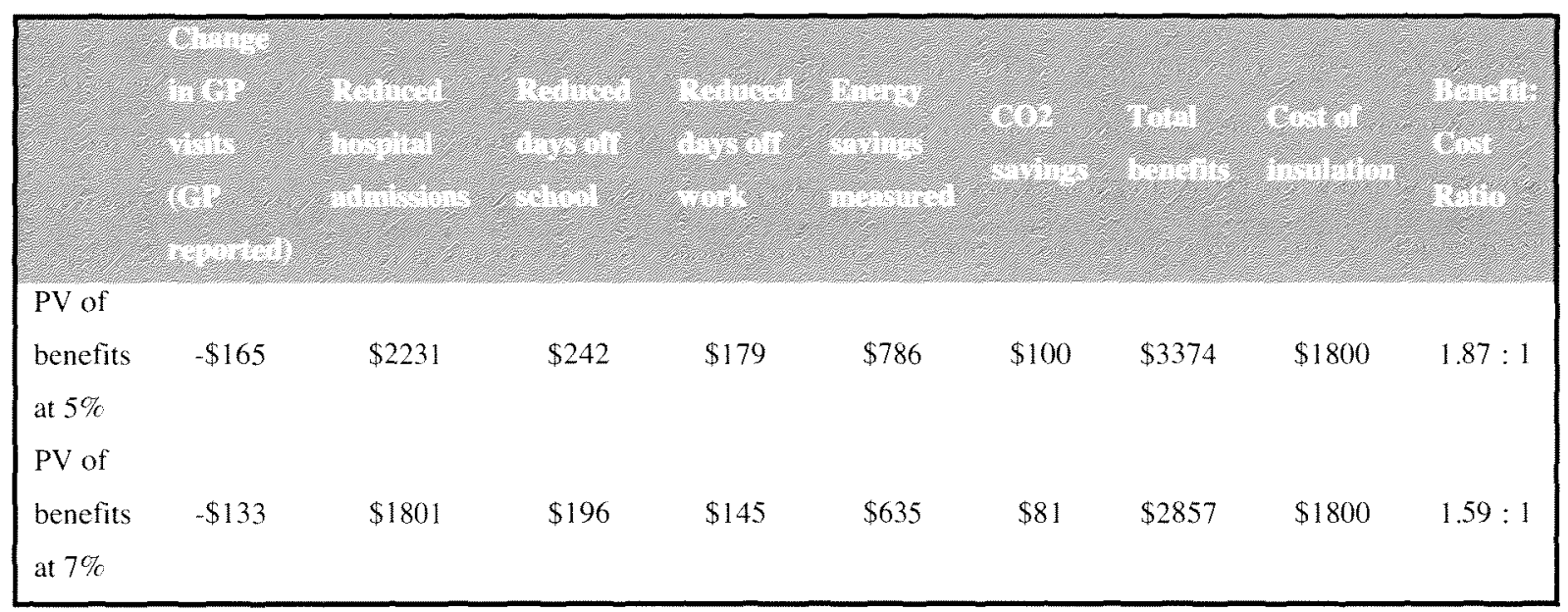

Source (Chapman et al., 2007, in review, p 7) 
This analysis naturally forms the starting point for a cost benefit analysis of the Housing, Heating and Health Study, the methodology of which is set out in the following chapter. 


\section{METHODOLOGY}

Chapter 3 sets out the conceptual framework, model and analytical approach that are the basis of the cost benefit analysis.

\subsection{Conceptual Framework}

The assumption that cost benefit analysis is a useful and appropriate tool for assessing interventions such as the Housing, Heating and Health Study is the foundation of the conceptual framework of this thesis.

Cost benefit analysis is a method of assessing the net present costs and benefits of an action or policy that can form an important part of the decision making process of organisations and governmental bodies. Cost benefit analysis is routinely used by governments throughout the world, although specific assumptions may vary greatly (Daly and Farley, 2004; MED, 2007). In general, this thesis follows the guidelines set out by the New Zealand Treasury (Treasury, 2005); this was a pragmatic decision based on the value of an approach consistent with standard New Zealand governmental practice; in any case, Treasury guidelines are generally consistent with international practice. As already noted, the cost benefit analysis of the Housing, Insulation and Health Study (Chapman et al., 2007, in review) was also an important starting point, given the similarities between that study and the Housing, Heating and Health Study.

The New Zealand Treasury suggests that cost benefit analysis should be undertaken from a national perspective, and should attempt to encompass all of the public and private benefits and costs of an action for the economy as a whole. To this end, transfer payments such as taxes, which redistribute wealth within the economy, are not included in cost benefit analysis. Fiscal costings such as depreciation, capital charges and accounting costs/benefits are also not included. Environmental and social costs and benefits should be included when it is possible to monetise them or otherwise quantify them (Treasury, 2005). This general approach appears to be consistent with standard analytic practice (US EPA, 1999; Clinch and Healy, 2001; Goodacre, Sharples and Smith, 2002, Access Economics, 2005; MED, 2007) 
There are a number of limitations inherent in cost benefit analysis that it is important to acknowledge:

Firstly, there are often a number of assumptions built into a given analysis resulting from the availability of information and the costs of obtaining more accurate information (Treasury, 2005). The significance of these assumptions can be explored by using sensitivity analysis to assess the degree to which they may affect the outcome of the analysis.

Secondly, despite the potentially comprehensive nature of cost benefit analysis, there may be intangible costs and benefits which it is not possible to value monetarily, but which are still important. These intangibles should be assessed, if possible, using other methods (Treasury, 2005) and should not be accorded less importance with regard to decision making (Arrow et al, 1996). It is important to exercise caution in drawing conclusions where significant costs or benefits cannot be monetised and included.

Thirdly, cost benefit analysis can not address ethical concerns, such as the impact of a proposed policy on social inequality (Heinzerling and Ackerman, 2002).

Finally, the results of a cost benefit analysis may give a false impression of concreteness; it is important to acknowledge that it is only one of a number of tools that can be used as part of the decision making process (Daly and Farley, 2004). There is no reason why quantifiable costs and benefits should be given more weight than ethical or other qualitative considerations.

\subsubsection{Discount rate}

A discount rate is a measure of the degree to which future benefits/costs are given weight in present day terms and allows analysts to estimate the "net present value" (NPV) of a future benefit/cost stream. If the NPV of a project or investment is greater than zero it is generally considered to be worthwhile and the calculation of NPV enables multiple potential projects to be compared and ranked. Discounting can be 
based on time preference, the opportunity cost of capital or the uncertainties associated with future costs/benefits; the selection of an appropriate discount rate is one of the philosophical issues at the heart of environmental economics because the rate chosen for a given piece of analysis may potentially have a large effect on the outcome. Analysis of intergenerational environmental problems such as pollution may be particularly affected, as over longer periods of time, particularly using higher discount rates, the present value of future environmental costs and benefits will be negligible (Daly and Farley, 2004; Rose, 2007; MED, 2007). The ethical issues associated with such intergenerational comparisons have led some theorists to suggest that cost benefit analysis is not an appropriate tool for assessing the value of government actions with social benefits (Heinzerling and Ackerman, 2002).

Establishing an appropriate discount rate for a given cost benefit analysis requires an assessment of the nature of the project/issue. Young (2002) details two main approaches for assessing government decisions, based on the social opportunity cost of capital and the social rate of time preference respectively. The social opportunity cost of capital approach is based on market interest rates, and has been the preferred method of the New Zealand Treasury which has used a discount rate of $10 \%$ real and suggested that lower discount rates should be used "only in exceptional circumstances" (Treasury, 2005, p 29). This approach has been criticised by a number of economists including Rose (2007). The social rate of time preference approach is based on the "marginal rate of substitution between consumption in one period and the next" (Young, 2002, p 4), and may be a more appropriate tool for assessing proposed activities with positive environmental externalities which may be underprovided by the market (Chapman et al., 2007, in review). Chapman et al. (2007, in review) thus adopt a $5 \%$ discount rate based on the social rate of time preference, which is consistent with international analyses of home energy efficiency improvements (Clinch and Healy, 2001; Goodacre, Sharples and Smith, 2002).

In line with the analyses cited above a discount rate of $5 \%$ has been adopted and a sensitivity analysis using discount rates of $2.5 \%$ and $10 \%$ is also carried out. 


\subsection{The Model}

The model developed for this cost benefit analysis is "three pronged" and was designed as a form of sensitivity analysis in order to explore the extent to which different assumptions about the future occupancy of intervention group homes may affect the outcome of the cost benefit analysis. The model was developed from the intuitive starting point that population mobility and household change may have some impact on any health related costs and benefits resulting from the intervention. For example, if it is the case that the intervention reduces the number of visits of the average asthmatic child to his/her GP, that reduction will be considered a benefit of the intervention and be included in the analysis. However, the analysis takes place over the predicted life-time of the study heaters and during that time the child will grow up, raising the question of what effect the child becoming an adult will have on the net health outcomes of his or her household.

More importantly, the model addresses the potential impact of Study households moving to new homes. New households will move into the Study homes who may have fewer asthmatic children or perhaps no children at all and thus the net health outcomes due to improved domestic space heating may be different. It is very likely that the Study heaters will remain in the same physical location; they are not easy to move and are costly to reinstall in the case of owner-occupiers and in the case of renters, the Study heaters belong to the landlord and will not move when the household does. On a more pragmatic note, if Study heaters were to move location it would be impossible to predict their continued impact given unknowns involved in new location such as the degree of insulation, so it is not useful to consider this possibility.

The model was designed to explore some of these difficulties. Three future scenarios can be modelled in order to establish to what extent changes in study home occupancy will affect the outcome of the analysis;

Model A is considered to be the most likely model. In this scenario, intervention group households will continue to occupy their homes for a period predicted by data on average length of home occupancy. When these households move, they will be 
replaced by new families with identical composition in terms of age, sex and number of members, but with rates of asthma that are consistent with average population rates. These families will in turn move after a period of time, but as they will be replaced by identically composed families this and any subsequent changes will not have any further impact on the analysis. Aging is not a factor in Model A.

One assumption behind the design of Model $\mathrm{A}$ is that it is intuitively reasonable to assume that a dwelling that is suitably sized for a given household is likely to continue to be occupied by similarly sized households. A literature search did not reveal any research that would allow the author to predict future home occupancy based on current occupancy, so this assumption is unsupported, and may possibly be questionable in the light of demographic trends which suggest that the average New Zealand household size may slightly decrease in the coming decades (Statistics New Zealand, 2005).

Model B is based on similar assumptions. The key difference between the two Models is that $\mathrm{B}$ is based on the assumption that future households will have the same proportion of asthmatic adults and children as current occupants and thus all asthma related health benefits will be unaltered by changes in occupancy. The value of Model $\mathrm{B}$ is that it is somewhat plausible to assume that future occupants of a given dwelling are likely to share some health traits with the current occupants; given, for example, the positive correlation between lower socioeconomic status and higher asthma rates (Basagaña et al., 2004) and between socioeconomic status and the standard and location of home that can potentially be purchased or rented. Basagaña et al. also conclude that households in areas with a low educational level have higher asthma rates than could be predicted given the socioeconomic status of the individual households. It is also reasonable to assume that families with asthmatic children may seek out homes with suitable heating, increasing the likelihood of similar families occupying the Study homes.

Model $\mathrm{C}$ is very different from the previous two and assumes that the current occupants will continue to occupy their respective homes for the entire period of the analysis. During that period Study children will eventually become adults (i.e. reach the age of 14 , defined as an adult in terms of the Study) and at that point will be 
treated as Study adults in terms of their health outcomes. It is assumed that asthmatic children will become asthmatic adults and that non-asthmatic children will become non-asthmatic adults. As the average age of Study children was ten, the model assumes that children will become adults after four years. Eventually children will generally leave home; however, for ease of analysis, and in the absence of an ability to model such changes, it is assumed that all current occupants will continue to occupy the Study dwelling for the period of analysis. It is assumed there will be no new household members. Additional health savings resulting from aging in adults cannot be explored, due to the low number of older participants in the study, which meant that older adult health data were not analysed separately. Model $\mathrm{C}$ is thus conservative in that conditions such as COPD and heart disease affect a higher proportion of older people, but the potential health savings from reductions in the impact of these conditions as a result of the intervention will not be captured. Scenario $\mathrm{C}$ is the least plausible scenario and is limited by a large number of potentially challengeable assumptions.

\subsubsection{Population mobility}

An important element of Model $\mathrm{A}$ is establishing an average length of occupation for owner-occupiers and tenants. Statistics New Zealand Census data provides a suggestive picture of occupancy patterns, but does not allow for the degree of precision that is necessary for the model. A review of published and grey literature established that the average length of rental tenure for New Zealand households is 15 months (HNZC, 2005). Renters are not a homogenous group and can be divided into several distinct categories (MfE, 2004); however, it is not possible to find a more detailed assessment of New Zealand renter mobility. It is more difficult still to establish an estimate of the average length of occupancy for owner-occupiers, with a figure of seven years the best estimate (Richards, 2007).

Using these figures as a starting point, it is important to address the possibility that the duration of occupancy might be effected by the installation of the Study heaters. This possibility is relevant to Model A as the eventual replacement of current Study households with non-Study households with average population levels of asthma may 
reduce the effect of any health related changes. A longer period of occupancy for the Study households, resulting from the installation of the study heaters, might thus increase the estimated benefits of the cost benefit analysis to some degree. No quantitative research was available that addressed this point directly, but there is suggestive evidence that improvements in home heating might have some impact on length of occupancy. Clark and Onaka (1983) summarise evidence from 18 surveys suggesting that on average $6 \%$ of households move as a result of issues to do with the quality or design of their homes, which suggests that improvements in home heating might slightly increase the average duration of household occupancy. The authors discuss the vast array of reasons why a household might move which include forced moves, concerns over a broader range of home characteristics, changes in employment and changes resulting from the life-cycle of the household e.g. childbirth or aging.

The MfE (2004) reported that, based on a survey of renters in Christchurch, coldness was an important reason for moving. It seems plausible that the improved temperatures reported in Howden-Chapman et al. (2008, in press), might induce households who rent to occupy their current homes for a longer period, particularly as they are aware of the connections between asthma and space heating. It is, however, less plausible to imagine that owner-occupiers might significantly increase their duration of occupancy as a result of improved heating, as decisions to move home are not made lightly given the high transaction costs incurred when selling and buying a home.

The 2005 Study Head of Household Questionnaire asked householders to estimate how long they had lived in their current dwelling. Analysis of the response to this question indicates that owner-occupiers in the intervention and control groups had occupied their homes for approximately 6.6 years, and renters 5.6 years. Assuming that in 2005 households were halfway through their period of occupancy this suggests a further occupancy period of 6.6 years for owner-occupiers and 5.6 years for renters. This is a much longer period in both cases than could be predicted based on the available research. It is unclear whether these figures reflect a selection bias e.g. relatively settled families might be more likely to join the Study because they might predict that they are more likely to occupy their homes long enough to gain benefit 
from the Study heaters, or whether these figures simply reflect the reduced mobility of households with children of a certain age (6-12).

Based on the research and figures cited above, and taking any impact that the new heaters might have in extending occupancy, an average further period of occupancy for Study households of four years from the time of the intervention is a reasonably conservative estimate. This is one of the assumptions underlying Model A. Following the replacement of Study households with households with average rates of asthma in Model A, length of occupancy is not relevant for the rest of the period of analysis. Length of occupancy data is also not relevant to Models B and C.

\subsubsection{Prevalence of asthma in New Zealand}

Scenario A is based on the replacement of current Study households with households with a proportion of asthmatic members equal to that found in the general population. In order to assess this proportion it is necessary to define asthma. There are a number of approaches that can potentially be used to assess the prevalence of asthma, including whether or not a person has experienced wheezing in past year (Asher et al., 2001); however the prevalence of wheezing in a population does not necessarily correspond with the prevalence of asthma, as some people experience occasional wheeze which may not be the result of asthma (Holt and Beasley, 2001). Other possible definitions include a certain level of bronchial hyper-responsiveness combined with current symptoms, which is considered an epidemiologically sound approach (Holt and Beasley, 2001).

The approach taken in this cost benefit analysis was largely dictated by the data that was collected as part of the study. Participants were asked a wide variety of questions concerning asthmatic symptoms, but the most straightforward means of categorising participants as asthmatic or non-asthmatic was their response to the question "has a doctor ever told you that you have asthma?" In order to make predictions about the future outcomes of average New Zealand asthma rate households in Model A it is thus necessary to use this definition of asthma to assess rates of asthma in the general population of New Zealand. 
A recent study by D'Souza et al. (1999) reported that $15.9 \%$ (13.8\%-17.9\%, 95\% C.I.) of adults questioned had ever been told by a doctor that they had asthma. This figure is reasonably consistent with a 1989 study of children in Hawkes Bay which reported that $13.3 \%$ of children studied had ever been told by a doctor they had asthma, a large increase from a related 1975 study which reported an 8\% rate (Shaw et al., 1990). Given that there is an increasing prevalence of asthma in New Zealand children (Holt and Beasley, 2001), it is reasonable to adopt $15.9 \%$ as an estimate of the proportion of all New Zealanders (adult or child) who have ever been told by a doctor that they have asthma.

Table 2 details the composition of an average Study household, a typical New Zealand household of the same size and composition, and a Study household after children have become adults: 


\begin{tabular}{|c|c|c|c|}
\hline & 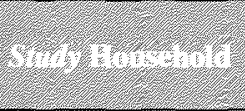 & 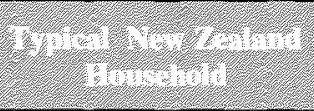 & (1) \\
\hline No. adults & 1.95 & 1.95 & 3.94 \\
\hline No. children & 1.99 & 1.99 & 0 \\
\hline Proportion of adults that are asthmatic & $37 \%$ & $15.9 \%$ & $55 \%$ \\
\hline $\begin{array}{l}\text { Proportion of children that are } \\
\text { asthmatic }\end{array}$ & $73 \%$ & $15.9 \%$ & $\mathrm{~N} / \mathrm{A}$ \\
\hline Total asthmatic adults per household & 0.72 & 0.31 & 2.16 \\
\hline Total non asthmatic adults & 1.23 & 1.64 & 1.77 \\
\hline Total asthmatic children & 1.44 & 0.32 & 0 \\
\hline Total non-asthmatic children & 0.54 & 1.67 & 0 \\
\hline
\end{tabular}

Sources: Head of Household Questionnaire, Adult's (14 years and over) Questionnaire and Child's (0 to 13 years) Questionnaire.

\subsubsection{Purchase, maintenance and replacement of domestic space heating}

Assumptions about the lifespan, maintenance and replacement of heaters form an important part of the cost benefit analysis.

The Housing, Heating and Health Study heaters were selected following an analysis by He Kainga Oranga (2005) which summarised information regarding heat output, outdoor emissions, indoor emissions and capital and operating costs for a number of heaters, and a tendering process (Howden-Chapman et al., 2008, accepted with revisions).The assessment by He Kainga Oranga (2005) established that a 20 year average life-span was a reasonable estimate for the Study heaters, and thus a 20 year horizon is the basis of the cost benefit analysis. A sensitivity analysis of the impact of life-span will be conducted by analysing outcomes over a ten year period.

The cost benefit analysis is based on the comparison of the net costs and benefits of the intervention and control groups, which will include the cost of maintaining and replacing heaters. Both groups reported using a variety of space heating devices during the winter months of 2006 with more than $60 \%$ of households reporting the use of multiple types. In order to make a comparison between the two groups in terms of maintenance and replacement costs it is necessary to simplify the comparison.

Question 16B of the 2006 Study Head of Household Questionnaire asked participants 
to indicate their main form of heating during the past winter, and it is practical to limit comparison to the results of this question. Table 3 summarises Q16B;

Table 3: Main Heater Used in 2006

\begin{tabular}{|lcc|}
\hline Open fire & \multicolumn{1}{|c|}{ (1) } \\
Enclosed fire & 1 & 1 \\
Wood pellet burner & 0 & 19 \\
Flued gas heater & 30 & 0 \\
Unflued gas heater & 4 & 0 \\
Heat pump & 2 & 59 \\
Electric heater & 138 & 0 \\
Central heating & 0 & 89 \\
Other what & 0 & 0 \\
None. & 0 & 0 \\
\hline Total & 0 & 0 \\
\hline
\end{tabular}

Source: Nevil Pierse, Study biostatistician and project manager

This data has been adjusted to take account of the fact that a small minority of control group participants reported the use of flued gas heaters or heat pumps, which is considered highly unlikely (post-questionnaire checks by Study researchers confirmed that some Study participants had difficulties in identifying their heater type). The data also reflects the complex realities of the intervention in that not all intervention group households made their Study heater their main heater; a number of participants in the intervention group reported problems with their Study heaters and others chose, for a variety of reasons, to make other heating decisions. It is reasonable to make the simplifying assumption that the comparison of the intervention and the control group is based on the Study heaters installed, given the likelihood that householders will learn how to use their heaters effectively. The heaters installed in the intervention group households are detailed in Table 4: 


\begin{tabular}{|ll|}
\hline & \\
Flued Gas Heater & 5 \\
Heat pump & 144 \\
Wood pellet burner & 30 \\
\hline
\end{tabular}

Source: Personal Communication, Louise Nicol, He Kainga Oranga

The basis for the comparison of the intervention and control groups is thus an assumption that over the period of analysis the main heater used in intervention dwellings will be the Study heater installed, and that control group homes will continue to use the same main heater recorded in Q16B. This is a necessary simplification, given that people's heating choices will fluctuate as a result of changes in circumstance, income and preference. In addition, when households move they will take portable domestic space heating devices with them and as new households enter the Study dwellings they will bring with them new domestic space heating devices and preferences.

If it is assumed that in 2006 non-Study heaters were halfway through their life-times we can predict when they will need to be replaced based on available data on average appliance lifespan. The Ministry for the Environment (MFE, 2005) compiled a detailed review of heating options which provides the basis for this analysis. Difficulties are raised by the fact that the categories available in Q16B of the 2006 Head of Household questionnaire such as "electric heater" can potentially encompass a wide range of electric heater types, such as convector panel and electric resistance based heaters, which have markedly different prices. However, it is reasonable to assume that the electric heaters used by the control group are relatively inexpensive and thus a conservative decision was made to select an average price towards to lower end of the cost spectrum. The data is presented in Table 5: 
Table 5: Heater Installation Cost and Average Life-span

\begin{tabular}{|c|c|c|c|c|}
\hline (n) & (n) & (3) & S & 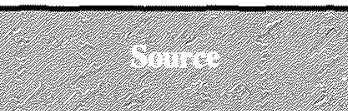 \\
\hline Electric Heater & $\$ 98.76$ & MFE (2005) & 10 & MFE (2005) \\
\hline Enclosed Fire & $\$ 2,765.16$ & MFE (2005) & 20 & MFE (2005) \\
\hline $\begin{array}{l}\text { Flued Gas } \\
\text { Heater }\end{array}$ & $\$ 2,864.70$ & $\begin{array}{c}\text { He Kainga } \\
\text { Oranga }\end{array}$ & 20 & He Kainga Oranga (2005) \\
\hline Heat Pump & $\$ 2,200$ & $\begin{array}{c}\text { He Kainga } \\
\text { Oranga }\end{array}$ & 20 & He Kainga Oranga (2005) \\
\hline Open Fire & N/A & N/A & Lifespan of house & $\begin{array}{c}\text { Authors simplifying } \\
\text { assumption }\end{array}$ \\
\hline $\begin{array}{l}\text { Unflued Gas } \\
\text { Heater }\end{array}$ & $\$ 296.27$ & MFE (2005) & 10 & MFE (2005) \\
\hline $\begin{array}{l}\text { Wood Pellet } \\
\text { Burner }\end{array}$ & $\$ 3516.66$ & $\begin{array}{c}\text { He Kainga } \\
\text { Oranga }\end{array}$ & 20 & He Kainga Oranga (2005) \\
\hline
\end{tabular}

Many types of domestic space heater require regular maintenance. Heat pumps require the regular cleaning of filters by owners in addition to annual service by a professional in order to continue functioning optimally (He Kainga Oranga, 2005). All heaters/fires with a flue require regular servicing for reasons of safety and efficiency and gas based heaters require regular maintenance in order to avoid gas leakage and to ensure optimal function (MoH, 2005). Annual maintenance for non electric heater types is typically recommended by central and local government agencies in New Zealand. Average maintenance costs were established by contacting a service provider in each of the four main Study communities and are detailed in Table 6 (certain assumptions such as single storey housing were made with regard to flue maintenance): 


\begin{tabular}{|lc|}
\hline Enclosed fire & \\
Flued gas heater & $\$ 57.78$ \\
Heat pump & $\$ 97.78$ \\
Open fire & $\$ 108.21$ \\
Pellet burner & $\$ 57.78$ \\
Unflued gas heater & $\$ 88.89$ \\
\hline
\end{tabular}

Source: Service providers.

Establishing an approach to the analysis of heater maintenance is an important part of the cost benefit analysis because of the connection between maintenance and optimal heater performance.

2006 Study data are based on the outcome of installing brand new (and presumably optimally functioning) heaters in intervention households, and comparing the health outcomes and energy use of these households with control group household outcomes that were presumably the result of using heaters that were on average half way through their lifespan and may have been operating at suboptimal performance levels if they were not maintained according to recommendations. Suboptimal control group heater performance is likely to be the case based on consultation with maintenance providers who suggest that it is rare that maintenance is carried out annually; when consulted many service providers assumed that the author had a perceived problem with a heater of the type discussed, indicating that a common pattern of behaviour is to wait for a problem such as noticeably poor performance, noises or, in the case of gas fuelled space heaters, odours, before requesting maintenance (personal communications, names withheld on request). Anecdotal evidence from industry sources also suggests that heater maintenance does not occur on a regular basis (Dr. Robyn Phipps, Senior Lecturer Massey University, personal communication). A literature review of academic and grey literature did not reveal any research on real world appliance maintenance behaviour.

If it is assumed that, on average, any decrease in energy efficiency or change in health impacts resulting from a lack of maintenance is linear over the lifetime of a space 
heating device, there is no difficulty in estimating the performance of non-Study heaters over the course of the 20 year period of analysis, as we can assume that the control group data collected in 2006 (when the heaters were on average halfway through their life-cycles) reflects average performance. However, it is not possible to make a similar assumption in the case of the Study heaters.

Thus, the difficulty faced, from an analytical perspective, is that the cost benefit analysis is based on a comparison of optimally functioning Study heaters which will function less optimally during the twenty year period of analysis without maintenance with heaters that have probably not been optimally maintained, but whose current performance is a reasonable basis for estimating future performance. A review of the available literature suggests it is not possible to estimate what effect decreasing Study heater performance might have on health and energy use related outcomes; however, we cannot assume that any change in intervention group outcomes as a result of the intervention will continue undiminished over the course of the analysis if Study heaters are not maintained.

The problem is explored by carrying out a sensitivity analysis, comparing intervention and control group costs based on three scenarios. The first assumes manufacturer/government recommended annual maintenance; the second assumes four yearly maintenance, and the third assumes non-maintenance. It is acknowledged that the second and third (non-annual) maintenance scenarios are limited because we cannot estimate the impact of any reduction in heater performance, which may exaggerate any health benefits and energy savings of the intervention. However, an assumption of annual maintenance is also clearly unrealistic. For this reason the four yearly maintenance scenario has been selected as the basis of the final cost benefit analysis. 


\subsubsection{Extrapolating winter data}

There is a strong case for extrapolating winter data to include cold days that occur during the non-winter period, particularly in the early spring and late autumn, when cold days are still likely and when heating behaviour is likely to be reasonably consistent with that which occurs in winter months e.g. fuel is likely to be available and behaviour patterns similar.

Chapman et al. (2007, in review) use a multiplier of 1.67 based on "degree day" data from BRANZ's Malcolm Cunningham to extrapolate energy savings and winter health costs and benefits in order to include cold days that occur outside the winter period in their analysis. The Housing, Insulation and Health Study analyses a winter period of three months (June, July and August), while the Housing, Heating and Health study analyses a four month winter period (June, July, August and September). Based on the assumption that all winter months are equally cold it is possible to convert the figure used by Chapman et al. (2007, in review) and a conservative multiplier of 1.25 can be calculated for use in the present analysis.

Following Chapman et al. (2007, in review), a smaller multiplier of 1.125 is appropriate when analysing days off school as the winter period defined by the Study encompasses a greater proportion of the total school days in a year.

\subsubsection{Statistical significance}

The statistical significance of results produced by analysing the Study data is an important consideration; typically a p-value below 0.05 for a given outcome (a $95 \%$ probability that that outcome is not the result of chance) is considered an appropriate criterion for statistical significance. However, in the case of the Housing, Heating and Health Study, which has a relatively small number of subjects, it is more appropriate to carry out a sensitivity analysis, comparing the net health use and energy consumption benefits/costs resulting from a range of $\mathrm{p}$-value criteria. The author decided, in consultation with members of He Kainga Oranga, that results based on 
three criteria for statistical significance, $p<0.05, p<0.10$ and $p<0.20$ should be analysed and contrasted, with the relatively low criterion for inclusion of $p<0.10$ ( $90 \%$ probability that an outcome is not the result of chance) being the basis for inclusion in the baseline scenario of the cost benefit analysis. This decision, while not conservative, is justified by the inherent limitations of housing intervention studies. Given the high cost of interventions of this type it is unlikely that future studies will be conducted that have sufficient statistical power to capture all of the benefits and costs resulting from an intervention at a $\mathrm{p}<0.05$ level, even if these benefits or costs genuinely resulted from the intervention in question; thus, from a policy perspective, it is important not to exclude benefits/costs from the present study which have a lower level of statistical significance.

\subsubsection{Analysis of data}

Analysis of the data is a reasonably straightforward procedure. The initial stage is to analyse whether any statistically significant change has occurred as a result of the intervention with respect to each separate category of person and health outcome, for example visits to GPs for chest problems by asthmatic adults, and to either include or discard data based on the statistical significance criterion chosen. A combined yearly health related benefit/cost figure resulting from the intervention can then be calculated for a typical asthmatic child, non-asthmatic child, asthmatic adult or non-asthmatic adult. Using these figures a total figure per household can then be calculated for the average Study household, typical New Zealand household, or Study household whose children have become adults as required. These figures can then be extrapolated over the appropriate horizon using the standard discounting formula and the appropriate discount rate, in accordance with the household occupancy predicted by the choice of Model. The NPV calculated can then be combined with the NPV of any energy use savings/costs, comfort benefits and care-giver related savings.

Finally, this figure can be contrasted with the additional purchase, replacement and maintenance costs resulting from the intervention for the same horizon and discount rate in order to calculate the NPV per household of the intervention. An example of 
the calculation is set out in Figure 1, based on Model B, a 20 year horizon, a 5\% discount rate and a statistical significance criterion of $p<0.10$.

Figure 1: An Example of the Calculation of the NPV of the Intervention per Household

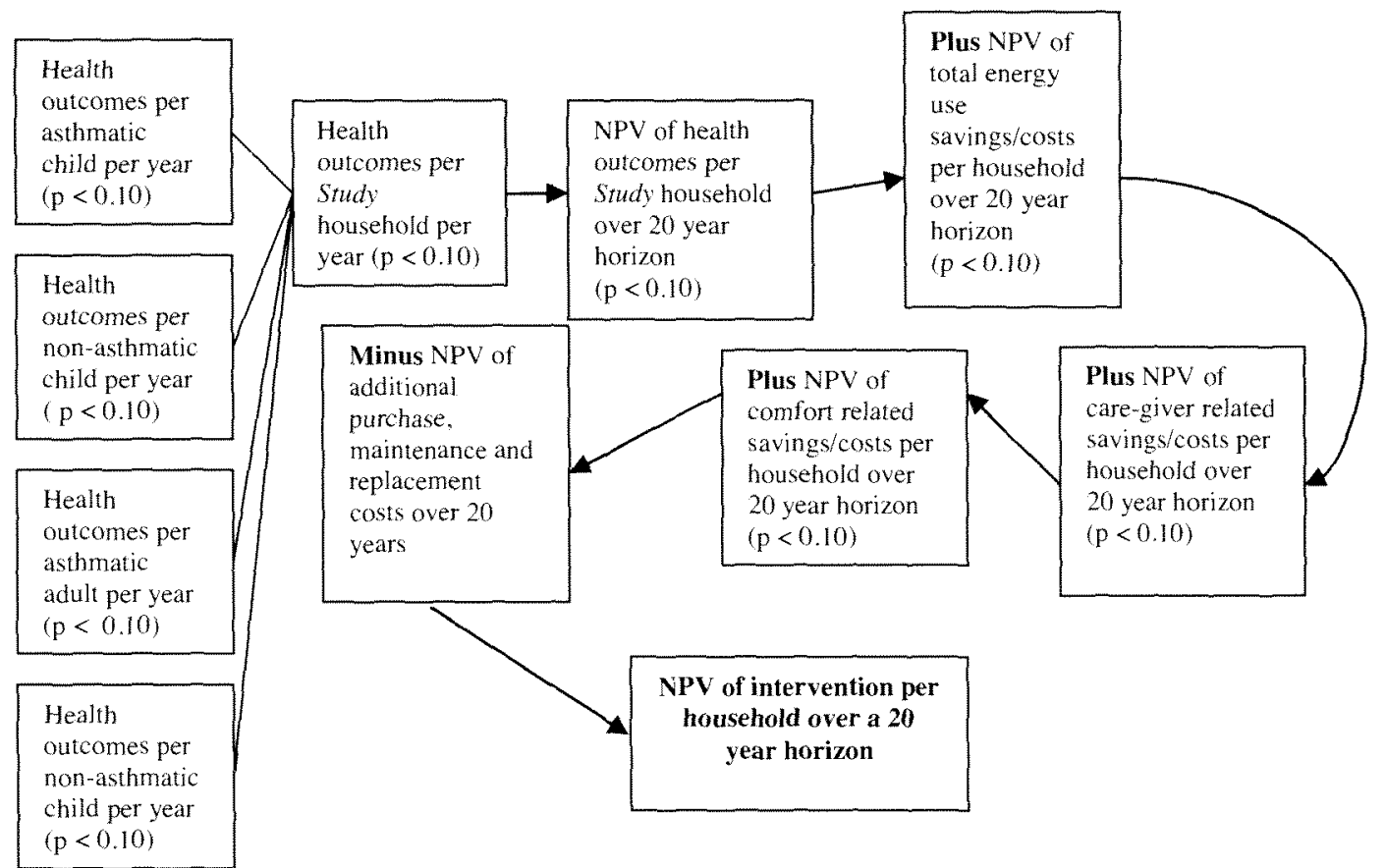

\subsection{Analysis of Available Data from the Housing, Heating and Health Study}

\subsubsection{Energy use}

Changes in energy use resulting from the intervention are a potentially important element of the cost benefit analysis. Energy use data were collected using fortnightly (2005) and monthly (2006) energy use questionnaires, and the Head of Household Questionnaire (2005). Electricity use data were also collected from the relevant power companies and compiled by He Kainga Oranga's data analyst.

There are two approaches to analyzing the available data: to assess whether any statistically significant change has occurred in the total energy costs and $\mathrm{CO}_{2}$ emissions of the intervention group households relative to the control group as a result of the intervention, or to focus on costs and emissions calculated based solely on power company data (which is more likely to be reliable, given the limitations of self 
reported data based on recall and estimation). The latter option is more conservative, but as the intervention involved, in many cases, replacing unflued gas heaters with electricity consuming heat pumps, an assessment which focuses solely on electricity use may produce distorted conclusions. Thus both sets of data will be analyzed, and total energy use will be the basis for the final analysis.

Costing energy use is straightforward for power company data (reported in kWh) using MED price data. It is also straightforward to cost self reported non-electricity energy use data from the monthly 2006 Study questionnaires as they include estimates of spending. However, the equivalent fortnightly data from 2005 are not available for analysis due to a poor response rate, so estimates of 2005 non-electricity energy use are based on energy use questions in the 2005 Head of Household Questionnaire, which required households to estimate the quantities of fuel consumed during the winter but did not require an estimate of spending, thus necessitating a more complex approach to calculation. 2005 spending on LPG was estimated based on an average price reported by the MfE (2005), spending on coal was based on a $\$ / \mathrm{kg}$ figure calculated from the 2006 monthly questionnaires as was spending on wood. In the case of wood it was necessary to use a different price for the control and intervention groups as there was a notable variation in the price per $\mathrm{m}^{3}$ (reflecting the fact that wood may sometimes be free). It is not necessary to provide a cost estimate for pellets used in 2005 as at that time no Study participants had pellet burners.

In order to analyze the energy use data, it is also necessary to calculate $\mathrm{CO}_{2}$ emissions per dollar for each fuel type. This was done using data from MED, MfE and carboNZero.

Key cost and emissions data are summarized in Table 7 below. It was not necessary to convert energy data into a standard format (e.g. kWh) in order to analyze them and thus data are presented in the units used in the analysis. 
Table 7: Energy Use Costs and Emissions per Unit

\begin{tabular}{|c|c|c|c|c|}
\hline 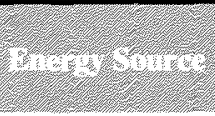 & and & 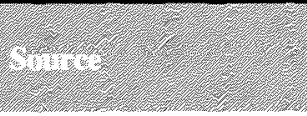 & (1) & 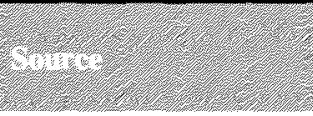 \\
\hline Electricity & $\$ 0.17 / \mathrm{kWh}$ & MED Energy Data File & $0.4 \mathrm{~kg} \mathrm{CO} 2 / \mathrm{kWh}$ & $\operatorname{MED}(2007)$ \\
\hline Reticulated gas & $\$ 0.10 / \mathrm{kWh}$ & MED Energy Data File & $0.19 \mathrm{~kg} \mathrm{CO} 2 / \mathrm{kWh}$ & www.carboNZero.co.nz \\
\hline LPG & $\$ 2.07 / \mathrm{kg}$ & MfE 2005 & $3 \mathrm{~kg} \mathrm{CO} 2 / \mathrm{kg}$ & MfE 2005 \\
\hline Coal & $\$ 0.45 / \mathrm{kg}$ & Study data & $2.77 \mathrm{~kg} \mathrm{CO} 2 / \mathrm{kg}$ & MfE 2005 \\
\hline $\begin{array}{l}\text { Wood } \\
\text { (intervention } \\
\text { group) }\end{array}$ & $\$ 39.13 / \mathrm{m}^{3}$ & Study data & $0 \mathrm{kgCO} 2 / \mathrm{m}^{3}$ & $\mathrm{MfE}(2007 \mathrm{~A})$ \\
\hline $\begin{array}{l}\text { Wood (control } \\
\text { group) }\end{array}$ & $\$ 58.22 / \mathrm{m}^{3}$ & Study data & $0 \mathrm{~kg} \mathrm{CO} 2 / \mathrm{m}^{3}$ & $\operatorname{MfE}(2007 \mathrm{~A})$ \\
\hline Pellets & $\begin{array}{l}\text { As per } \\
\text { data }\end{array}$ & Study data & $0 \mathrm{~kg} \mathrm{CO} 2 / \mathrm{kg}$ & $\mathrm{MfE}(2007 \mathrm{~A})$ \\
\hline
\end{tabular}

Emissions factors were generally not difficult to assess, however, the emission factor chosen for electricity consumption is potentially controversial. Because New Zealand has a high proportion of renewable energy, its average emissions factor for electricity generation is a relatively low $0.23 \mathrm{~kg} \mathrm{CO}_{2}-\mathrm{e} / \mathrm{kWh}$ (MED, 2007). However, it is more appropriate to assess changes in energy use in terms of marginal emissions factors, which reflect the reality of energy generation. Marginal emissions factors are based on the assumption that electricity generated from renewable sources such as hydrodams will be used first, while non-renewable coal and gas based generation will “accommodate changes in electricity demand" (MED, 2007, p 2.4). Marginal emissions factors are based on $\mathrm{CO}_{2}$-e emissions from a more fossil fuel intensive mix of electricity sources and are therefore higher than average emissions factors. The cost benefit analysis of the Housing, Insulation and Health Study was based on a marginal emissions factor of $0.63 \mathrm{~kg} \mathrm{CO}_{2}$-e $/ \mathrm{kWh}$ (Chapman et al., 2007, in review). More recently, however, an analysis by the Ministry of Economic Development has indicated that, while a figure of $0.6 \mathrm{~kg} \mathrm{CO}_{2}-\mathrm{e} / \mathrm{kWh}$ is appropriate for assessing marginal emissions for the period 2006-2010, beyond that point predicted increases in renewable energy mean that a lower figure of $0.4 \mathrm{~kg} \mathrm{CO}_{2}$-e $/ \mathrm{kWh}$ is more appropriate (MED, 2007).

\footnotetext{
${ }^{2}$ As burning pellets does not cause net $\mathrm{CO}_{2}$ emissions (following $\mathrm{MfE}$ (2007A)), and it was not necessary to estimate the cost of any pellets used in 2005 (as none were used) it was not necessary to calculate a $\$ /$ bag figure for pellets. Pellet use was costed based on the spending reported by pellet burner users in the 2006 monthly questionnaires.
} 
For reasons of simplicity, the lower figure of $0.4 \mathrm{~kg} \mathrm{CO}_{2}-\mathrm{e} / \mathrm{kWh}$ has been adopted for this cost benefit analysis, as the majority of the period of analysis falls beyond 2010 . This can be considered a conservative decision as it may marginally underestimate $\mathrm{CO}_{2}$ emissions savings from the intervention.

It is also necessary to assign a financial value to any change in $\mathrm{CO}_{2}$-e emissions resulting from the intervention. This is a difficult task given the high degree of volatility in the emissions trading markets and the broad range of marginal social costs that have been estimated (Tol, 2005; Sinclair, 2006). Chapman et al. (2007, in review) use a price of $\$ 30 \mathrm{NZ}$ per tonne based on the then current EU Emissions Trading Scheme EUA (EU allowances) price of EUR 20-30 per tonne. This price has since undergone a sharp decline (Stern, 2007). Sinclair (2006), in a report commissioned by the New Zealand Treasury, suggests that these prices are an artifact of the EU-wide emissions cap and are thus not appropriate for New Zealand based assessments, and that it is more reasonable to estimate the cost of emissions based on Certified Emission Reductions (CERs), the market value of which the author estimates at US $9.65 / \mathrm{tCO}_{2}$-e $(\$ 14.85)$. It is important to note that both EUA and CER prices result from emissions trading based on the reductions required of Annex One nations during the first commencement period of the Kyoto Protocol; these reductions are relatively modest, given that in order to curb global warming it is likely to be necessary to reduce developed countries annual emissions by around $80 \%$ (Stern, 2007) or more (Hansen, 2008).

The social benefit of a reduction of $\mathrm{CO}_{2}$-e emissions by one tonne is thus potentially much greater and has been estimated to be as high as US $85 \mathrm{tCO}_{2}-\mathrm{e}$ (year 2000 prices) (Stern, 2007). Tol (2005) has reported a broad range of estimates with a mean of US $\$ 28 / \mathrm{t} \mathrm{CO} 2$-e (year 2000 prices). Social benefit estimates vary as a result of multiple factors including discount rate and risk modeling (Stern, 2007).

In the context of such a high degree of uncertainty, it seems reasonable to use the $\$ 14.85$ estimate of Sinclair (2006), as this is based on a relatively conservative approach. Sources such as MED (2007) predict that beyond 2012 a higher figure will be appropriate; however the current analysis takes a more conservative approach for reasons of simplicity. 
Household energy cost and emissions data is normally distributed and linear regression is carried out using the $\mathrm{R} 2.4 .1$ package. Following a review of energy use analysis in previous interventions (Shortt and Rugkasa (2007); Oreszczyn et al. (2006)), and an assessment of the available data, the following potential confounding variables were initially included in the regression analysis:

- Number of people in the household

- Low income level reported

- No. of people under 5 in the household

- No. of people over 65 in the household

- Previous year's energy use

- Thermal drapes

- General condition of house

- Geographic location of household

- Age of the house

It was considered necessary to assess these factors, despite control and intervention groups having been assessed as generally very similar (Howden-Chapman et al., 2008 , accepted with revisions), because usable energy data were only available for a subset of Study households $(\mathrm{n}=176)$, and it was important to avoid any distortion of outcomes. It is important to note that the Study was designed primarily to assess the impact of the intervention on asthma related outcomes, and as such, there is a limit to the statistical power of the Study in terms of analyzing energy data.

\subsubsection{Health care utilisation}

Changes in health care utilisation are a potentially important benefit resulting from the intervention: index child data, previously analysed, suggested a statistically significant reduction in parentally reported GP visits (Howden-Chapman et al., 2008, accepted with revisions). Health data were collected for all Study participants and are available for analysis. In accordance with the model it is possible to analyse health care utilisation data by dividing householders into four categories: asthmatic child, non-asthmatic child, asthmatic adult and non-asthmatic adult. 
The health data collected in the Health Questionnaires include a variety of useful information regarding mental and physical health; however, the most easily costed data were those relating to health care utilisation. Questions quantifying usage of the following health services were included in the questionnaires (in some cases separate questions were asked about respiratory and non-respiratory related visits):

- GP visits

- Talks with nurses for health care or advice (respiratory related only)

- Talks with pharmacists for health care or advice (not including prescription collection, respiratory related only)

- Visits to after-hours clinics

- Emergency room visits

- Admissions to hospital

- Appointments with public hospital specialists/ clinics

- Visits to private specialist doctors.

At the present time, official GP record data is not available for analysis, and hospital record data were not collected (personal communication, Nevil Pierse, project manager and biostatistician).

In order to carry out any analysis of this data it is first necessary to establish costs for the different health services utilised. An initial literature review suggested that such costs are not readily available at a national level (Access Economics, 2005); there is no government organisation that compiles average health costs, and contact with the New Zealand Health Information Service confirmed that, while this information may be available at a District Health Board level, it is not available at a national level.

It is thus necessary to review published and grey literature in order to estimate costs. A starting point is the cost benefit analysis carried out by Holt and Beasley (2001), which includes estimates of the cost of asthma related hospitalisation and emergency room visits ( $\mathrm{p}$ 36). Chapman et al. (2007, in review) assess the resource cost of GP visits, and their figure is adopted. Aish et al. (2003) provide a useful source of cost estimates from a South Auckland health intervention, including costs for after hours A\&M (accident and medical) clinic visits and registered nurse consultation. A 
literature review did not reveal a useful means of estimating the resource cost of a non prescription related visit to a pharmacist for health care or advice. Intuitively a potential measure would be based on the length of an average consultation and a calculation of the value of a pharmacist's time in terms of average salary and hours worked; however, the uncertainties surrounding this type of calculation mean that it not worth pursuing. The remaining health care services to be costed; specialist private doctor visits, and visits to public hospital specialists and clinics are more difficult to estimate. In order to produce a cost estimate for appointments at public hospitals with respiratory specialists, contact was made with an expert at the Capital \& Coast District Health Board (confidential personal communication). An estimate of the resource cost of private specialist doctors was not possible and thus the figure calculated by Chapman et al. (2007, in review) for GPs was used; this is a plausible and conservative estimate. The results of the costing process are summarised in Table 8:

Table 8: Health Care Utilization Costs

\begin{tabular}{|c|c|c|}
\hline GP visit & $\$ 49.18$ & Chapman (2007 in review) \\
\hline After hours clinic & $\$ 41.63$ & Aish et al. (2003) \\
\hline $\begin{array}{l}\text { Admission to emergency } \\
\text { ward - respiratory }\end{array}$ & $\$ 227.43$ & Holt and Beasley (2001) \\
\hline $\begin{array}{l}\text { Public hospital specialist or } \\
\text { clinic-chest }\end{array}$ & $\$ 310.18$ & $\begin{array}{l}\text { Personal communication } \\
\text { CCDHB (2008) }\end{array}$ \\
\hline Private specialist doctor & $\$ 49.19$ & Chapman ( 2007 , in review) \\
\hline $\begin{array}{l}\text { Hospital admission - chest } \\
\text { (age } 0-19 \text { ) }\end{array}$ & $\$ 1299.94$ & Holt and Beasley (2001) \\
\hline $\begin{array}{l}\text { Hospital admission - chest } \\
\text { (age 20-69) }\end{array}$ & $\$ 1609.97$ & Holt and Beasley (2001) \\
\hline $\begin{array}{l}\text { Hospital admission - chest } \\
\text { (age } 70+\text { ) }\end{array}$ & $\$ 2931.45$ & Holt and Beasley (2001) \\
\hline
\end{tabular}

All figures were adjusted to 2006 levels using the "Health and Community Services, Inputs" element of the Producer Price Index (Statistics NZ).

It is not unreasonable to use the figures cited above to analyse the cost of non-chest related health care utilisation. It is certainly reasonable to analyse visits to GPs, specialist doctors and after-hours clinics, given the connection between mental health and dampness/mould and the connection between mental health and increased community health care usage (Layard et al., 2006) and the fact that utilisation costs do not vary based on the health problem in question. However, it is less reasonable to 
analyse non-chest related hospitalisation, hospital clinic visits and emergency room visits given the difficulty in assigning costs and the limited evidence of a connection between serious non-chest related health problems and improved space heating. Following this reasoning any change in non-chest related hospital admissions and visits to public hospital specialist or clinics will not be included in the analysis.

All analysis of medical use data was carried out using the $\mathrm{R} 2.4 .1$ package, and was analysed using generalised linear models with the logistic and Poisson log link, as per the analysis of index child data presented in Howden-Chapman et al. (2008, accepted with revisions). Data were adjusted for sex, age, ethnicity, parental income, region, smoking in the home and $\mathrm{NO}_{2}$ levels in the child's bedroom and baseline measures if available. Data entry and statistical modelling were carried out by He Kainga Oranga; the author had little input into this part of the analysis, other than in decisions relating to the model used in the cost benefit analysis.

Other medical visit related benefits of the intervention, such as potential reductions in transport use for trips to the doctor, are not assessed. Reductions in transport time have a number of benefits including reduced fuel costs and reductions in harmful emissions.

\subsubsection{Medication use}

Prescription data for index children were collected from GPs' records as part of the data collection process; however the data requires analysis by a qualified medical professional and at the time of writing this has not occurred (Nevil Pierse, personal communication). A secondary source of data is the Child's (0 -13) Questionnaire, which contains questions about asthma related non-inhaled steroid use and chest related antibiotic use which have the potential to be analysed. The Adult's (14 and over) Questionnaire did not contain detailed questions regarding medication use, so any changes in adult medication use cannot be assessed.

Winter non-inhaled steroid use data reported in the Child's $(0-13)$ Questionnaire was more detailed; however discussion with a number of pharmacies (Johnsonville, 
Hataitai) lead to the conclusion that the three brands used by Study children have a similar cost, with a suggested value of $\$ 6.35$ (adjusted to 2006 prices using the PPI) for an average five day course of Betnesol, Prednisone or Redipred.

The Child's (0-13) Questionnaire also included a question regarding winter chest related antibiotic use. However, it did not require parents to specify the type of chest related antibiotic used by their child and thus an assessment of the costs of antibiotic use requires a certain degree of approximation. A recent cost benefit analysis of community acquired pneumonia in New Zealand estimated that a typical course of antibiotics for pneumonia (Amoxycillin clavulate) and paracetemol cost $\$ 28.78$ (adjusted to 2006 prices using the PPI) (Scott et al., 2004), and this provides a reasonable basis for costing changes in antibiotics use in the absence of more specific information.

No useable inhaled asthma medication data was available from the Child's Health (013) Questionnaire; however, the use of inhaled asthma medication was recorded in the index children's daily health diaries, and the results were reported in HowdenChapman et al. (2008, accepted with revisions). This provides a good proxy for analysis of the use, by all asthmatic children, of the medications Flixotide (Fluticasone) and Ventolin (Salbutamol) which were selected as typical examples of the categories "preventer" and "reliever" in order to assign a cost to usage. The cost of these medications can be estimated via PHARMAC data as they are both fully funded, based on the assumption that all index children aged six and over are members of a Primary Health Care Organisation (PHO) and assuming a 10\% wholesaler mark-up of the manufacturers price (PHARMAC, 2007; personal communication, Hataitai Pharmacy, 2008).

A single puff of Flixotide was estimated to cost $\$ 0.03$, while a puff of Ventolin was estimated to cost $\$ 0.05$. The change in daily puffs is analysed and then extrapolated to include the entire winter (approximately 120 days).

\subsubsection{Days off school/paid work due to illness}


Reductions in days off school or days off work are an important potential benefit of the intervention. Days off school/work for adults (14 and older) were recorded in the Adult Health Questionnaires. There are two potential sources of days off school data. The Child's (0-13) Questionnaire included a question regarding the number of days off school due to illness, and school record data for index children was also sourced directly from schools as part of a Masters thesis by Sarah Free (Howden-Chapman, 2008 , accepted with revisions). Data based on official records will be used in the final analysis and assumed to apply to all asthmatic children, as it is it is more likely to be accurate. All days off school/work data was processed using R 2.4.1 as per health service utilisation data.

Costing days off work and school requires an assessment of lost production from the perspective of society. Chapman et al. (2007, in review) base their assessment of the value to society of a day of paid work lost on $2 / 3$ of the average gross daily wage, which can be considered reasonable given the potential of co-workers to "pick up some of the slack" in the short term. Other recent assessments of the value of a day off work have been based on the average gross wage (Access Economics, 2005B; Jakob, Craig and Fisher, 2006) suggesting that this approach is conservative. Based on an average gross hourly wage in 2006 of \$22.24 (Statistics New Zealand, 2007A), following the approach used by Chapman et al. (2007, in review), the loss of productivity resulting from a sick day is roughly $\$ 111.20$.

The value of a day off school for a teenager is estimated to be $2 / 3$ of the daily gross minimum wage ( $\$ 41.00$, based on the 2006 youth minimum wage of $\$ 8.20$ ) and the value of a day off school for a child at primary school half this figure (Chapman et al., 2007 , in review). Intuitively, it is reasonable to assume that additional days of school due to illness may also have a longer term negative impact on children's educational achievement which could potentially be costed; however, research by McNaughton et al. (1993) discussed in Biddulph, Biddulph and Biddulph (2003) suggests that while asthmatic children do take more days off school than average, this does not have an impact on their long term reading ability. Taras and Potts-Datema (2005) carry out a comprehensive review of the connections between asthma, school attendance and achievement and conclude that two thirds of published studies "demonstrate no 
difference in levels of academic achievement or ability" in asthmatic children as a result of additional days off school (p 304).

It is also important to note that opportunity costs resulting from the loss of parent/care-giver time due to children's illness may not be captured by the analysis (except in the case of working adults who may possibly have included days off work looking after their sick children when reporting days off work due to illness).

Research by Laforest et al. (2004) suggests that days off school for asthmatic children may have a significant economic cost in terms of care-giver absenteeism. As there is no Study data directly addressing the question of care-giving for sick children this issue is addressed in Section 3.4.4 as one of several issues beyond the direct scope of the data.

\subsection{Issues beyond the Direct Scope of the Data}

There are a number of potential costs and benefits that cannot be assessed by a direct analysis of the available Study data. This section briefly discusses some of these costs and benefits, and the methodological issues around attempting to quantify them.

\subsubsection{The value of improved comfort and mental health}

It is difficult to assess the value of any changes in the comfort of Study participants, as concepts such as comfort are nebulous and difficult to define in practice. Clinch and Healy (2001) and Goodacre, Sharples and Smith (2002) measure comfort in their respective models by calculating the difference between the maximum potential energy saving that households could theoretically make following energy efficiency improvements and the actual saving that they are predicted to make assuming a $30 \%$ take-back effect following research by Milne and Boardman (2000).

Such an approach can be taken with regard to the Housing, Heating and Health Study by assuming that any reductions in energy usage as a result of the intervention represent $70 \%$ of an intervention household's maximum potential savings. This approach can be considered reasonable because average temperatures in control 
households during the winter of 2006 were not far from the $16.5^{\circ} \mathrm{C}$ that was the basis of Milne and Boardman (2000)'s assessment. A calculation of the value of comfort is made on this basis and is reported in Chapter 4 (Section 4.4). A sensitivity analysis is carried out based on a 10\% take-back effect and a $50 \%$ take-back effect and the outcomes are reported in Section 5.1.3.

It is important to note the limitation of such an approach, which is that if the data indicates that no energy savings have occurred as a result of the intervention, perversely comfort benefits cannot be estimated. As we know that the intervention has resulted in an increase in indoor temperatures, it certain that this will result in improved comfort, however difficult this is to value.

Improved mental health is a potential outcome of improved domestic space heating; however, mental health is a broad category and presents serious problems from an analytic perspective. SF36 questions asked as part of the Study included questions regarding mood, energy levels and changes in social activities. However, as Chapman et al. (2007, in review) concluded with regard to similar data from the Housing, Insulation and Health Study, there is insufficient data to make an economic assessment of any changes in these variables.

Improvements in mental health result in reduced healthcare utilisation for both physical and mental complaints (Layard et al., 2006). In so far as improved mental health results in fewer visits to GPs or specialist doctors any mental health benefit of the intervention will be captured by the analysis of study participants' utilisation of such health services. Visits to mental health professionals may have been captured by the Study question regarding non-chest related visits to private specialist doctors, but it is unclear whether participants would have included visits to mental health professionals in this category. Reduced days off work or school are also a potential consequence of improved mental health (Layard et al., 2006), and these are captured by the analysis outlined in Section 3.3.4. Potential changes in other medical costs such as reductions in quantities of psychiatric drugs prescribed cannot be assessed and neither can other possible benefits such as increased employment, reduced stress or harm for family members, lost leisure time and reduced police/judicial costs. 
Chapman's (2007) discussion of the health costs of leaky buildings provides one potential basis for assessing mental health related costs that cannot be measured via the Study data. However, the cost of mental illness that the author calculates includes medical costs which must be ignored by the current analysis in order to avoid double counting. Focussing only on the value of the QALYs (quality adjusted life years) lost per case of mental illness, and adjusting for a different cost structure, it is possible to estimate a New Zealand cost of $\$ 4,137$ per year (Associate Professor Ralph Chapman, personal communication).

Given that there is an odds ratio of 1.4:1 for mental illness for occupants of structurally flawed dwellings relative to the general population (Weich and Lewis, 1998) it would, thus, theoretically be possible to estimate the value of reductions in damp/mould in Study dwellings as a result of the intervention in terms of proportionate reductions in QALYs lost to mental illness. However, assessing changes in mould and damp is not a simple matter, as there are a number of different potential measures which may not always produce consistent outcomes (Mikael Boulic, PhD candidate, Massey University, personal communication). Research examining a 40 dwelling subset of Study dwellings suggests that the intervention resulted in a statistically significant reduction in relative humidity (which relates to dampness). Mould related results are difficult to assess, with no statistically significant change in the presence of xerophilic or saprophytic fungi according to air sampling results, but a statistically significant reduction in xerophilic fungi according to dust samples and in both types according to slide results (Boulic and Phipps, 2008).

It is not possible to draw conclusions about the value of any reduction in QALYs lost to mental illness that might occur as a result of the changes in dampness and mould reported above, although it seems likely that some benefit will have occurred.

Analysis of health care utilisation, days off work data and increases in comfort based on the take-back effect provides a simplistic but useful method for capturing the mental health and comfort related benefits of the intervention. The inability of the current analysis to capture any reduction in QALYs lost to mental illness as a result of the intervention means that the analysis may be conservative. The baseline scenario for the cost benefit analysis will include comfort related benefits. 


\subsubsection{The value of new cases of asthma avoided}

There is some evidence that exposure to damp/mouldy environments may increase the odds of developing asthma. The IOM (2004), which conducted a comprehensive review of the health effects of exposure to damp or mouldy indoor environments, concluded that there was "limited or suggestive evidence of an association" between the development of asthma and exposure to damp indoor environments. Fisk, Gomez and Mendell (2007), drew similar conclusions, calculating an odds ratio of 1.34 for asthma development in damp/mouldy homes based on four studies, with a $95 \%$ confidence interval that included unity.

The possibility that improving domestic space heating might result in fewer household members developing asthma suggests potentially large benefits to the economy during the lives of those people. A life-span based analysis of the cost of asthma has not been carried out in New Zealand; however, a U.S. EPA (1999) assessment estimated direct medical costs alone of approximately NZ $\$ 24,000$ (converted to 2006 prices using the "Health and Community Services, Inputs" element of the Producer Price Index) discounted at a 5\% rate over the lifetime of an average patient.

This figure does not include indirect costs and reflects a different cost structure; however, it is suggestive. Given the limited nature of the evidence of a connection between asthma development and dampness/mould, and the difficulties associated with analysing such changes, any such cost cannot be included in the cost benefit analysis. Future research may clarify the connection between asthma development and damp and mould, enabling a more comprehensive cost benefit analysis of reductions in damp and mould to be undertaken at that time.

\subsubsection{Excess mortality avoided}

Excess winter mortality, as discussed in Chapter 2, may potentially be linked with domestic space heating via indoor temperature and harmful emissions. No 
information is currently available regarding any mortality that may have occurred in Study households, but it is unlikely, given the small size of the Study population, and the small proportion of household members aged 65 and over, that a statistically significant effect could be captured were this data available. A similar conclusion was reached as part of the cost benefit analysis of the Housing, Insulation and Health Study, which was based on a much greater number of participants (Chapman et al., 2007 , in review). Exclusion of any mortality related costs makes the cost benefit analysis slightly more conservative.

\subsubsection{The value of caregiver days off work}

As noted in Section 3.3.2, data on caregiver days off work looking after sick children were not collected, although it is possible that some Study participants may have included such days off work when asked the question "did you take days off work/school due to illness?" If it is assumed that this did not occur often, then it is possible to estimate the value of such care-giver time. White, Lavoie and Nettleman (1999) make such an estimate with regard to the costs relating to influenza vaccination in the United States. Following their basic methodology, it is possible to analyse the additional cost of a day off school. If we assume that care-giving will primarily be undertaken by mothers, we can then use labour force participation data showing that $62 \%$ of mothers were employed in 2001 (33\% full-time and $29 \%$ parttime) (Johnston, 2005). Using figures from Statistics New Zealand's Quarterly Employment Survey that show that the average woman earned $\$ 144.09$ per day (a part-time day of work is valued by the author at half this figure) (Statistics New Zealand, 2006) and applying Chapman et al. (2007)'s 2/3 reduction to account for coworkers "picking up the slack" and a 1.125 multiplier to account for cold days outside the winter period it is possible to estimate the additional caregiver cost of a child's day off school:

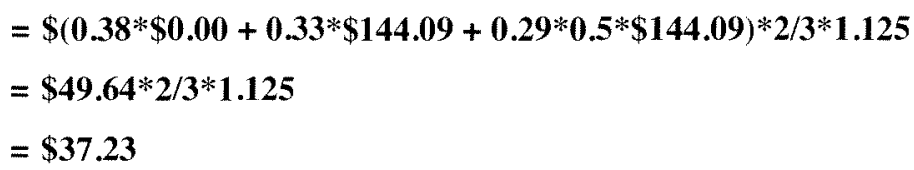

This figure is a simplistic one and does not take account the complicating fact that in some cases partners, relatives and friends who are not in paid employment may 
provide care-giving (although this will still entail an opportunity cost). It was not practical to assess the availability of such support. Despite the uncertainty surrounding such benefits the baseline scenario for the cost benefit analysis will include this data.

\subsubsection{The value of reduced damp and mould related repair costs}

Mould is an unsightly condition, and damp and mouldy houses have a characteristic musty and unpleasant smell. Extreme cases, such as the mould and dampness associated with "leaky building syndrome", can result in hundreds of thousands of dollars worth of repair bills (Consumer Build, 2008A). However, there is no evidence from a literature review that suggests that this degree of damage might be avoided by improving domestic space heating.

Minor mould and damp can be addressed by home owners by cleaning with a suitable product or in some cases by painting. It is likely, however, that if damp and mould in a home becomes significant it will eventually require professional repair work (personal communication, Mikael Boulic, 2008), although it is difficult to estimate the repair costs associated with damp and mould because they will vary greatly from case to case. Reductions in repair costs due to dwellings being drier and warmer are an additional benefit that could potentially be included in the analysis. A literature review did not suggest an appropriate method for estimating repair costs, or predicting what proportion of households might choose to undertake repairs. Given these difficulties it is necessary to ignore any possible reductions in repair costs that might result from the intervention.

\subsubsection{The potential impact of summer air conditioning using heat pumps}

When analysing changes in energy use and $\mathrm{CO}_{2}$ emissions following the intervention, a complicating factor is the possibility that any annual savings made during winter (and on cold non-winter days) could be reduced by the potential of heat-pumps to be used as air conditioners on warm days. Historically, only a very low proportion of New Zealanders use air conditioning, relative to other OECD nations such as the United States, which is likely to be a result of New Zealand's relatively temperate 
climate (Buckett, 2007). However, predicted increases in average temperature resulting from global warming may alter behaviour patterns, as might the availability of an air conditioning option. It is difficult to predict to what extent households may take advantage of this potential; two CEA surveys of a small sample of Christchurch homes which had heat pumps installed found that, over the course of two summers using the new technology, the proportion of homes reporting use of heat pumps for cooling on "the hottest of days" increased from 17\% to 57\% (Walker, 2004; Fyfe, 2005). Given that it is unlikely that the households in question had access to air conditioning prior to the installation of heat pumps, this change suggests that the occupants have developed a new expectation of summer comfort (Buckett, 2007), and is consistent with theories of thermal comfort creep discussed by Critchley et al. (2004). 60\% of respondents in a recent BRANZ study stated that they used their heat pump for cooling (French, 2008).

The impact of these complicating factors will be slightly mitigated, in the context of the intervention, by the fact that $20 \%$ of intervention group households did not have a heat pump installed. As electricity use data was collected for the entire period of the study, it would potentially be possible to compare the electricity use of these households during the summer months with control group electricity use data for the same period, using the methodology set out in section 3.3.1. However, possible gains such as lower heat related morbidity or improved comfort resulting from air conditioning cannot be estimated due to a lack of summer health data and thus summer electricity usage is not included in the analysis for reasons of consistency.

\subsubsection{Embodied costs}

The embodied costs of an appliance such as a heat pump include the environmental harm caused by emissions of $\mathrm{CO}_{2}$ as a by-product of the production and transport of the good, and may also include an estimate of the environmental harm caused by the extraction of the raw materials from which the device is made, depending on which measure is chosen. Methods such as life-cycle cost analysis also include costs associated with the disposal of a product. There is no single agreed framework for assessing embodied costs, and more importantly, there is no source of embodied cost 
data that could be used in the current cost benefit analysis in order to assess the relative embodied costs of Study and non-Study domestic space heaters. The lack of information regarding variables such as the make and origin of non-Study heaters mean that such an analysis is entirely beyond the scope of the available data.

\subsection{8 $\mathrm{Non}_{\mathrm{CO}_{2}}$ external emissions from electricity generation}

As discussed previously, domestic space heating can result in external emissions of environmentally harmful gases and particulate matter, either from the home directly via the chimney, windows and doors, or at the point of generation, in the case space heaters powered by electricity from coal and gas fired power stations.

There are a number of complications which limit the potential estimation of the health cost of external emissions at the household level. For example, factors such as the wetness of wood (which influences the efficiency of burning), the type of coal and the age of the heating appliance (older enclosed fires produce greater levels of external emissions than their modern counterparts) and the behaviour of occupants (opening of windows etc.) effect the quantity of harmful non- $\mathrm{CO}_{2}$ external emissions per $\mathrm{kWh}$ of energy produced by space heaters (ECAN, 2007; MfE, 2005). Although information regarding some of these factors was collected as part of the Study, the limits of the data and the complexity of analysis that would be required mean that it is reasonable to ignore the potential benefits of reduced external emissions at a local level. This decision is also justified by the fact that among the criteria for the inclusion of households in the Study were that the households use either an plug-in electric heater or an unflued gas heater as their main heat source, and these heaters are not a major source of local external emissions relative to open and enclosed fires (MfE, 2005).

It is relatively practical, however, to estimate the impacts of external emissions resulting from electricity generation. An analysis of the environmental costs of electricity generation in the Waikato region was recently commissioned by the Environment Waikato that included an assessment of the environmental cost of generation-related $\mathrm{SO}_{2}$ and $\mathrm{NO}_{\mathrm{x}}$ emissions (Denne, 2007). The potential health effects of $\mathrm{NO}_{\mathrm{x}}$ emissions on respiratory health have been discussed previously and $\mathrm{SO}_{2}$ has 
been associated with direct harm to the respiratory and cardiovascular systems (Denne, 2007, p 9). $\mathrm{SO}_{2}$ can also combine with water vapour and other molecules to form acid rain, which can cause a number of environmental harms; however this is not a significant issue in New Zealand currently (Denne, 2007). Denne concluded that there was not sufficient evidence of a causal relationship to include the direct health effects of $\mathrm{NO}_{\mathrm{x}}$ and $\mathrm{SO}_{2}$ emissions in his analysis, but that there was reason to assess the health effect of the sulphate and nitrate aerosols they can form. The health related costs established (with a high degree of acknowledged uncertainty), are presented in the following Table 9 in terms of $\$ / M W h$;

Table 9: Health Cost of Non- $\mathrm{CO}_{2}$ External Emissions from Electricity Generation in \$/MWh

\begin{tabular}{|lcccc|}
\hline Low Cost & & & & \\
Scenario & $\mathrm{SO}_{2}$ & $\$ 4.03$ & $\$ 0.00$ & $\$ 0.00$ \\
& $\mathrm{NO}_{\mathrm{x}}$ & $\$ 1.57$ & $\$ 0.70$ & $\$ 0.50$ \\
\hline $\begin{array}{l}\text { High Cost } \\
\text { Scenario }\end{array}$ & $\mathrm{SO}_{2}$ & $\$ 20.36$ & $\$ 0.00$ & $\$ 0.00$ \\
& $\mathrm{NO}_{x}$ & $\$ 7.91$ & $\$ 3.54$ & $\$ 2.52$ \\
\hline
\end{tabular}

Source: Denne $(2007, \mathrm{p}$ ii)

The marginal approach to the calculation of emissions factors discussed in 3.3.1 would be an appropriate means to assess the change in $\mathrm{SO}_{2}$ and $\mathrm{NO}_{\mathrm{X}}$ emissions resulting from a $1 \mathrm{kWh}$ reduction in electricity usage; however, such analysis is complex in practice and is beyond the scope of this thesis. Based on the average proportion of total electricity generated using coal and gas respectively in June 2006 (MED Energy Data File), and assuming that the figures above are representative of typical coal and gas based electricity generation in New Zealand, it is possible to calculate a health benefit per kWh saved of $\$ 0.0009$ following the low cost scenario and $\$ 0.005$ following the high cost scenario. It is clear that these figures are so low that they can be ignored in terms of the current analysis. 


\section{RESULTS}

This chapter presents the results of the cost benefit analysis of the Housing, Heating and Health Study. All tables include outcomes for a range of discount rates, statistical significance criteria and horizons. All benefits of the intervention are expressed as positive figures and all costs of the intervention are expressed as negative figures. For ease of understanding, in some tables, data that is significant at a p-value $<0.05$ level is coloured red, data that is significant at a p-value $<0.10$ level is coloured yellow, and data that is significant at a p-value $<0.20$ level is coloured green. In other tables scenarios or values of interest are highlighted in blue.

Figure 2: Key

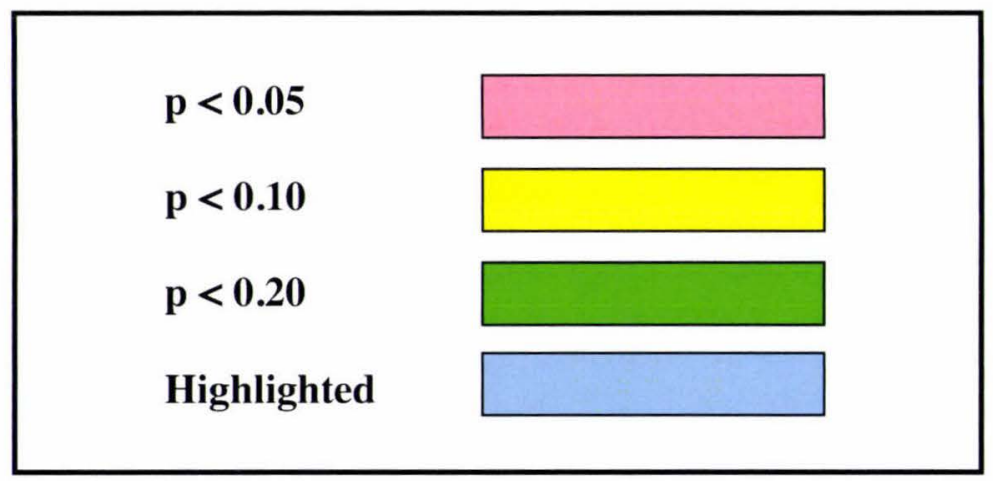

\subsection{Cost of Heater Purchase, Maintenance and Replacement}

Purchase, maintenance and replacement costs include the initial purchase and installation of the Study heaters. The figures reported in Table 10 below represent the NPV of the difference between the yearly heater purchasing, maintenance and replacement costs per average intervention group household and per average control group household. Three different maintenance scenarios are included as are results over both 10 and 20 year horizons. For example, the total additional purchase, maintenance and replacement costs of the intervention, assuming a 20 year horizon, maintenance every four years and a discount rate of $5 \%$ are $\$ 2,234.44$ (highlighted). 
Table 10: NPV of Change in Heater Purchasing, Maintenance and Replacement Costs per Intervention Group Household Following the Intervention

\begin{tabular}{|c|c|c|c|c|c|c|}
\hline $\begin{array}{l}\text { Discount } \\
\text { rate }\end{array}$ & \multicolumn{2}{|c|}{ Yearly maintenance } & \multicolumn{2}{|c|}{$\begin{array}{c}\text { Maintenance every four } \\
\text { years }\end{array}$} & \multicolumn{2}{|c|}{ Non maintenance } \\
\hline & $\begin{array}{l}10 \text { year } \\
\text { horizon }\end{array}$ & $\begin{array}{l}20 \text { year } \\
\text { horizon }\end{array}$ & $\begin{array}{l}10 \text { year } \\
\text { horizon }\end{array}$ & $\begin{array}{l}20 \text { year } \\
\text { horizon }\end{array}$ & $\begin{array}{l}10 \text { year } \\
\text { horizon }\end{array}$ & $\begin{array}{l}20 \text { year } \\
\text { horizon }\end{array}$ \\
\hline $\mathrm{r}=0.025$ & $-\$ 2772.58$ & $-\$ 3237.41$ & $-\$ 2176.18$ & $-\$ 2234.88$ & $-\$ 2056.73$ & $-\$ 1948.76$ \\
\hline $\mathrm{r}=0.05$ & $-\$ 2753.23$ & $-\$ 3075.20$ & $-\$ 2225.52$ & $-\$ 2264.72$ & $-\$ 2124.73$ & $-\$ 2049.52$ \\
\hline $\mathrm{r}=0.10$ & $-\$ 2716.40$ & $-\$ 2877.43$ & $-\$ 2293.59$ & $-\$ 2312.46$ & $-\$ 2221.58$ & $-\$ 2184.14$ \\
\hline
\end{tabular}

Table 10 demonstrates that the choice of discount rate does not alter the cost of the intervention to a great degree for a given maintenance scenario; this reflects that fact that the initial purchase and installation of the Study heaters were by far the greatest costs of the intervention and are not affected by discounting as they took place in year zero of the analysis. It is also clear from a comparison of the 10 and 20 years horizons for a given combination of discount rate and maintenance scenario that the choice of time-frame does not have a large impact on the total for similar reasons. The only significant difference is between maintenance scenarios, with the yearly maintenance scenario costing between on average $\$ 1000$ more than the no maintenance scenario over 20 years, and $\$ 790$ more than the four year maintenance scenario over 20 years. It is thus clear that the yearly maintenance model is the most conservative model, and, for the reasons discussed in Section 3.2.3, the four year maintenance scenario represents the most plausible case.

\subsection{Benefits of Changes in Health Care Utilisation, Medication Use and Days off School and Work}

Reductions in index children's health care utilisation, medication use and days off school are an established benefit of the intervention. This section sets out the benefit evaluation of the intervention per asthmatic child, non-asthmatic child, asthmatic adult and non-asthmatic adult. Adjusted health care utilisation, days off work/school, antibiotic and non-inhaled steroid use data are presented on a per winter basis, and inhaler use on a per day basis; all figures are converted to a full year figure using the appropriate multiplier (Tables $11-14$ ). Following this the results per household and the NPV of the total health related impacts of the intervention are presented (Table $15)$. 
Table 11: Value of Health Outcomes per Asthmatic Child as a Result of the Intervention

\begin{tabular}{|c|c|c|c|c|c|c|c|c|}
\hline & MRR & LCL & UCL & $\begin{array}{c}\text { p - } \\
\text { value }\end{array}$ & $\begin{array}{l}\text { Control } \\
\text { group } \\
\text { mean }\end{array}$ & $\begin{array}{l}\text { Change } \\
\text { resulting from } \\
\text { the } \\
\text { intervention } \\
\text { (3 d.p.) }\end{array}$ & $\begin{array}{l}\text { Reduction in } \\
\text { cost per child } \\
\text { per winter }\end{array}$ & $\begin{array}{c}\text { Reduction in } \\
\text { cost per child } \\
\text { per year }\end{array}$ \\
\hline $\begin{array}{l}\text { Days off school } \\
\text { (school record data) }\end{array}$ & & & & 0.04 & & -1.8 & $\$ 38.70$ & $\$ 43.54$ \\
\hline Visits to GP for chest & 0.7 & 0.55 & 0.89 & 0 & 1.23 & -0.369 & $\$ 18.18$ & $\$ 22.73$ \\
\hline Visits to GP other & 0.86 & 0.69 & 1.07 & 0.19 & 1.1 & -0.154 & $\$ 7.59$ & $\$ 9.49$ \\
\hline Nurse for chest & 0.88 & 0.5 & 1.54 & 0.65 & 0.35 & -0.042 & & \\
\hline Clinic for chest & 0.92 & 0.49 & 1.71 & 0.79 & 0.12 & -0.01 & & \\
\hline Clinic for other & 0.62 & 0.31 & 1.24 & 0.18 & 0.14 & -0.053 & $\$ 2.22$ & $\$ 2.77$ \\
\hline A \& E for chest & 2.23 & 0.97 & 5.14 & 0.06 & 0.05 & 0.062 & $-\$ 13.99$ & $-\$ 17.48$ \\
\hline Admitted for chest & 1.05 & 0.36 & 3.09 & 0.93 & 0.03 & 0.002 & & \\
\hline $\begin{array}{l}\text { Appointment for } \\
\text { chest }\end{array}$ & 0.54 & 0.21 & 1.38 & 0.2 & 0.07 & -0.032 & & \\
\hline Specialist for chest & 3.09 & 0.06 & 150.71 & 0.57 & 0 & 0 & & \\
\hline Specialist for other & 1.14 & 0.45 & 2.9 & 0.78 & 0.04 & 0.006 & & \\
\hline $\begin{array}{l}\text { Courses of non } \\
\text { inhaled steroids }\end{array}$ & 0.79 & 0.58 & 1.09 & 0.16 & 0.26 & -0.055 & $\$ 0.35$ & $\$ 0.43$ \\
\hline Courses of antibiotics & 0.8 & 0.61 & 1.05 & 0.11 & 0.35 & -0.07 & $\$ 2.01$ & $\$ 2.52$ \\
\hline No. Reliever puffs & 0.677 & 1.032 & 0.444 & 0.07 & 1.042226 & -0.337 & $\$ 2.02$ & $\$ 2.52$ \\
\hline No. Preventer puffs & 1.091 & 1.749 & 0.681 & 0.717 & 1.557771 & 0.142 & & \\
\hline $\begin{array}{l}\text { Total reduction in } \\
\text { health related costs } \\
\text { per child per year } p \\
<0.05\end{array}$ & & & & & & & & $\$ 66.27$ \\
\hline $\begin{array}{l}\text { Total reduction in } \\
\text { health related costs } \\
\text { per child per year p } \\
<0.10\end{array}$ & & & & & & & & $\$ 51.31$ \\
\hline $\begin{array}{l}\text { Total reduction in } \\
\text { health related costs } \\
\text { per child per year } p \\
<0.20\end{array}$ & & & & & & & & $\$ 66.52$ \\
\hline
\end{tabular}


Table 12: Value of Health Outcomes per Non-asthmatic Child as a Result of the Intervention

\begin{tabular}{|c|c|c|c|c|c|c|c|c|}
\hline & MRR & LCL & UCL & p - value & $\begin{array}{l}\text { Control } \\
\text { group } \\
\text { mean }\end{array}$ & $\begin{array}{l}\text { Change } \\
\text { resulting } \\
\text { from the } \\
\text { intervention } \\
(3 \mathrm{~d} . \mathrm{p} .)\end{array}$ & $\begin{array}{l}\text { Reduction } \\
\text { in cost } \\
\text { per child } \\
\text { per } \\
\text { winter }\end{array}$ & $\begin{array}{l}\text { Reduction } \\
\text { in cost } \\
\text { per child } \\
\text { per year }\end{array}$ \\
\hline Days off school & 1.18 & 0.83 & 1.67 & 0.37 & 3.14 & 0.565 & & \\
\hline Visits to GP for chest & 2.12 & 1.01 & 4.46 & 0.05 & 0.24 & 0.269 & $-\$ 13.25$ & $-\$ 16.56$ \\
\hline Visits to GP other & 0.88 & 0.59 & 1.32 & 0.54 & 0.84 & -0.101 & & \\
\hline Nurse for chest & 0.99 & 0.26 & 3.79 & 0.98 & 0.12 & -0.001 & & \\
\hline Clinic for chest & 0.9 & 0.35 & 2.34 & 0.83 & 0.04 & -0.004 & & \\
\hline Clinic for other & 1.3 & 0.46 & 3.68 & 0.62 & 0.07 & 0.021 & & \\
\hline A \& $E$ for chest & 4.45 & 0.27 & 73.85 & 0.3 & 0.01 & 0.035 & & \\
\hline Admitted for chest & 1 & 1 & 1 & 0.36 & 0 & 0 & & \\
\hline $\begin{array}{l}\text { Appointment for } \\
\text { chest }\end{array}$ & 0 & 0 & 0 & 0 & 0.03 & -0.03 & $\$ 9.31$ & $\$ 11.63$ \\
\hline Specialist for chest & 1 & 1 & 1 & 0.37 & 0 & 0 & & \\
\hline $\begin{array}{l}\text { Other service for } \\
\text { chest }\end{array}$ & $2.09 \mathrm{E}+09$ & 0 & Infinite & 0.99 & 0 & 0 & & \\
\hline Specialist for other & 1.35 & 0.29 & 6.31 & 0.7 & 0.04 & 0.014 & & \\
\hline $\begin{array}{l}\text { Courses of non } \\
\text { inhaled steroids }\end{array}$ & 2.04 & 0.44 & 9.57 & 0.37 & 0.04 & 0.042 & & \\
\hline Courses of antibiotics & 1.06 & 0.52 & 2.15 & 0.88 & 0.17 & 0.01 & & \\
\hline $\begin{array}{l}\text { Total reduction in } \\
\text { health related costs } \\
\text { per child per year, } p \\
<0.05\end{array}$ & & & & & & & & $\$ 11.63$ \\
\hline $\begin{array}{l}\text { Total reduction in } \\
\text { health related costs } \\
\text { per child per year, } p \\
<0.10\end{array}$ & & & & & & & & $-\$ 4.92$ \\
\hline $\begin{array}{l}\text { Total reduction in } \\
\text { health related costs } \\
\text { per child per year, } p \\
<0.20\end{array}$ & & & & & & & & $-\$ 4.92$ \\
\hline
\end{tabular}


Table 13: Value of Health Outcomes per Asthmatic Adult as a Result of the Intervention

\begin{tabular}{|c|c|c|c|c|c|c|c|c|}
\hline & MRR & LCL & UCL & P - value & $\begin{array}{l}\text { Control } \\
\text { group } \\
\text { mean }\end{array}$ & $\begin{array}{l}\text { Change } \\
\text { resulting from } \\
\text { the } \\
\text { intervention } \\
\text { ( } 3 \text { d.p.) }\end{array}$ & $\begin{array}{l}\text { Reduction } \\
\text { in cost per } \\
\text { adult per } \\
\text { winter }\end{array}$ & $\begin{array}{c}\text { Reduction } \\
\text { in cost per } \\
\text { adiult per } \\
\text { year }\end{array}$ \\
\hline GP Visits chest & 0.73 & 0.5 & 1.06 & 0.1 & 0.91 & -0.246 & $\$ 12.11$ & $\$ 15.13$ \\
\hline GP Visits other & 0.93 & 0.63 & 1.37 & 0.71 & 1.06 & -0.074 & & \\
\hline Nurse for chest & 0.81 & 0.39 & 1.67 & 0.56 & 0.3 & -0.057 & & \\
\hline A/H clinic for chest & 1.01 & 0.23 & 4.48 & 0.99 & 0.03 & 0 & & \\
\hline A/H clinic for other & 0.92 & 0.33 & 2.53 & 0.87 & 0.1 & -0.008 & & \\
\hline$A \& E$ for chest & 1.03 & 0.31 & 3.45 & 0.96 & 0.03 & 0.001 & & \\
\hline Admitted for chest & 2.56 & 0.31 & 21.35 & 0.39 & 0.01 & 0.016 & & \\
\hline Appointment for chest & 6.14 & 1.78 & 21.2 & 0 & 0.02 & 0.103 & $-\$ 31.89$ & $-\$ 39.86$ \\
\hline Specialist for chest & 0 & 0 & Infinite & 0.99 & 0.04 & -0.04 & & \\
\hline Specialist for other & 0.59 & 0.2 & 1.75 & 0.35 & 0.06 & -0.025 & & \\
\hline Days off & 1.17 & 0.82 & 1.67 & 0.39 & 3.45 & 0.587 & & \\
\hline $\begin{array}{l}\text { Total reduction in health } \\
\text { related costs per adult per } \\
\text { year, } p<0.05\end{array}$ & & & & & & & & $-\$ 39.86$ \\
\hline $\begin{array}{l}\text { Total reduction in health } \\
\text { related costs per adult per } \\
\text { year, } p<0.10\end{array}$ & & & & & & & & $-\$ 39.86$ \\
\hline $\begin{array}{l}\text { Total reduction in health } \\
\text { related costs per adult per } \\
\text { year, } \mathrm{p}<0.20\end{array}$ & & & & & & & & $-\$ 24.72$ \\
\hline
\end{tabular}

Table 14: Value of Health Outcomes per Non-asthmatic Adult as a Result of the Intervention

\begin{tabular}{|c|c|c|c|c|c|c|c|c|}
\hline & MRR. & LCL & UCL & p-value & Mean & $\begin{array}{l}\text { Change } \\
\text { resulfing } \\
\text { from the } \\
\text { intervention } \\
(3 \text { did. }) \text {. }\end{array}$ & $\begin{array}{l}\text { Reduction } \\
\text { in cost per } \\
\text { adnit per } \\
\text { winter }\end{array}$ & $\begin{array}{l}\text { Reduction } \\
\text { in cost per } \\
\text { adult per } \\
\text { year }\end{array}$ \\
\hline GP Visits chest & 0.81 & 0.46 & 1.4 & 0.45 & 0.24 & -0.046 & & \\
\hline GP Visits other & 0.87 & 0.61 & 1.22 & 0.41 & 0.88 & -0.114 & & \\
\hline Nurse for chest & 0.78 & 0.24 & 2.53 & 0.68 & 0.07 & -0.016 & & \\
\hline A/H clinic for chest & 0.29 & 0.04 & 1.86 & 0.19 & 0.01 & -0.007 & $\$ 0.30$ & $\$ 0.37$ \\
\hline A/H clinic for other & 1.47 & 0.62 & 3.51 & 0.39 & 0.04 & 0.019 & & \\
\hline A\&E for chest & 1.48 & 0.27 & 8.06 & 0.65 & 0.02 & 0.01 & & \\
\hline Admitted for chest & 27.07 & 11.86 & 61.77 & 0 & 0 & 0 & $\$ 0.00$ & $\$ 0.00$ \\
\hline Appointment for chest & 2.81 & 0 & 2513.1 & 0.77 & 0.01 & 0.018 & & \\
\hline Specialist for chest & 1.97 & 0.83 & 4.69 & 0.13 & 0 & 0 & $\$ 0.00$ & $\$ 0.00$ \\
\hline Specialist for other & 1.12 & 0.46 & 2.75 & 0.8 & 0 & 0 & & \\
\hline Days off & 0.88 & 0.56 & 1.37 & 0.56 & 3.09 & -0.371 & & \\
\hline $\begin{array}{l}\text { Total reduction in health } \\
\text { related costs per adult per } \\
\text { year }, p<0.05\end{array}$ & & & & & & & & $\$ 0.00$ \\
\hline $\begin{array}{l}\text { Total reduction in health } \\
\text { related costs per adult per } \\
\text { year, } p<0.10\end{array}$ & & & & & & & & $\$ 0.00$ \\
\hline $\begin{array}{l}\text { Total reduction in health } \\
\text { related costs per adult per } \\
\text { year, } \mathrm{p}<0.20\end{array}$ & & & & & & & & $\$ 0.37$ \\
\hline
\end{tabular}

Table 15: Value of Health Outcomes per Household as a Result of Intervention for each Model 


\begin{tabular}{|c|c|c|c|c|}
\hline & & $p<0.05$ & $p<0.10$ & $p<0.20$ \\
\hline \multirow{3}{*}{\multicolumn{2}{|c|}{$\begin{array}{l}\text { Yearly savings per Study household } \\
\text { Yearly savings per average population asthma rate household } \\
\text { Yearly savings per Study household, when children become adults }\end{array}$}} & $\$ 73.01$ & $\$ 42.52$ & $\$ 75.78$ \\
\hline & & $\$ 28.27$ & $-\$ 4.16$ & $\$ 6.00$ \\
\hline & & $-\$ 86.09$ & $-\$ 86.09$ & $-\$ 52.75$ \\
\hline & p-value & $\begin{array}{l}\text { Discount } \\
\text { rate }\end{array}$ & $\begin{array}{l}10 \text { year } \\
\text { horizon }\end{array}$ & $\begin{array}{l}20 \text { year } \\
\text { horizon }\end{array}$ \\
\hline \multirow[t]{9}{*}{ NPV of health savings for Model A } & \multirow[t]{3}{*}{$\mathrm{p}<0.05$} & $\mathrm{r}=0.025$ & $\$ 415.74$ & $\$ 609.05$ \\
\hline & & $\mathrm{r}=0.05$ & $\$ 376.94$ & $\$ 510.97$ \\
\hline & & $\mathrm{r}=0.10$ & $\$ 315.53$ & $\$ 382.51$ \\
\hline & \multirow[t]{3}{*}{$\mathrm{p}<0.10$} & $\mathrm{r}=0.025$ & $\$ 139.21$ & $\$ 110.75$ \\
\hline & & $\mathrm{r}=0.05$ & $\$ 133.41$ & $\$ 113.68$ \\
\hline & & $\mathrm{r}=0.10$ & $\$ 122.42$ & $\$ 112.56$ \\
\hline & \multirow[t]{3}{*}{$\mathrm{p}<0.20$} & $\mathrm{r}=0.025$ & $\$ 315.04$ & $\$ 356.09$ \\
\hline & & $\mathrm{r}=0.05$ & $\$ 293.78$ & $\$ 322.24$ \\
\hline & & $\mathrm{r}=0.10$ & $\$ 258.07$ & $\$ 272.29$ \\
\hline \multirow[t]{9}{*}{ NPV of health savings for Model B } & \multirow[t]{3}{*}{$\mathrm{p}<0.05$} & $\mathrm{r}=0.025$ & $\$ 638.95$ & $\$ 1,138.10$ \\
\hline & & $\mathrm{r}=0.05$ & $\$ 563.73$ & $\$ 909.81$ \\
\hline & & $\mathrm{r}=0.10$ & $\$ 448.59$ & $\$ 621.54$ \\
\hline & \multirow[t]{3}{*}{$P<0.10$} & $\mathrm{r}=0.025$ & $\$ 372.18$ & $\$ 662.92$ \\
\hline & & $\mathrm{r}=0.05$ & $\$ 328.36$ & $\$ 529.95$ \\
\hline & & $\mathrm{r}=0.10$ & $\$ 261.29$ & $\$ 362.03$ \\
\hline & \multirow[t]{3}{*}{$P<0.20$} & $\mathrm{r}=0.025$ & $\$ 663.23$ & $\$ 1,181.34$ \\
\hline & & $\mathrm{r}=0.05$ & $\$ 585.15$ & $\$ 944.38$ \\
\hline & & $\mathrm{r}=0.10$ & $\$ 465.63$ & $\$ 645.15$ \\
\hline \multirow[t]{9}{*}{ NPV of health savings for Model C } & \multirow[t]{3}{*}{$\mathrm{P}<0.05$} & $\mathrm{r}=0.025$ & $-\$ 154.97$ & $-\$ 743.60$ \\
\hline & & $\mathrm{r}=0.05$ & $-\$ 100.63$ & $-\$ 508.76$ \\
\hline & & $\mathrm{r}=0.10$ & $-\$ 24.68$ & $-\$ 228.64$ \\
\hline & \multirow[t]{3}{*}{$\mathrm{P}<0.10$} & $\mathrm{r}=0.025$ & $-\$ 269.64$ & $-\$ 858.27$ \\
\hline & & $\mathrm{r}=0.05$ & $-\$ 208.72$ & $-\$ 616.84$ \\
\hline & & $\mathrm{r}=0.10$ & $-\$ 121.31$ & $-\$ 325.26$ \\
\hline & \multirow[t]{3}{*}{$\mathrm{P}<0.20$} & $\mathrm{r}=0.025$ & $\$ 21.85$ & $-\$ 338.80$ \\
\hline & & $\mathrm{r}=0.05$ & $\$ 48.44$ & $-\$ 201.62$ \\
\hline & & $\mathrm{r}=0.10$ & $\$ 83.30$ & $-\$ 41.67$ \\
\hline
\end{tabular}


For Models A and B, for all statistical significance criteria, discount rates and horizons there are health savings on a per household basis. The lower outcomes for Model A in comparison with Model B are the result of the reduced proportion of asthmatic children in Model A households after the Study households are modelled as moving. Model $\mathrm{C}$, is the exception, with negative health savings for all probability criteria, discount rates and time periods; this reflects the fact that according to the data the intervention resulted in additional costs per asthmatic adult, and thus the benefits accrued per asthmatic child became a loss when the children became adults - a surprising result.

Another surprising result of the data and modelling is that owing to the p-value of various health outcomes, there is a greater positive benefit as a result of the intervention (at a household level) at a $\mathrm{p}<0.05$ level of statistical significance than at a $p<0.10$ level for all Models. This means that, perversely, the choice of $p<0.10$ for the baseline analysis leads to reduced health outcomes relative to a more stringent $p<$ 0.05 criterion.

Analysis of the outcomes for asthmatic and non-asthmatic children and adults indicates that by far the largest positive impact of the intervention was on asthmatic children, with an annual health related saving per child of $\$ 66.27$ (significant at a $\mathrm{p}<$ 0.05 level).

\subsection{Energy Use}

Table 16 sets out any energy and electricity use related savings on a per household basis, and the NPV of these savings for a variety of statistical significance criteria, discount rates and horizons. 
Table 16: Total Energy and Electricity Savings per Household as a Result of Intervention

\begin{tabular}{|c|c|c|c|c|c|c|}
\hline & $\begin{array}{l}\text { Saving over full } \\
\text { year }\end{array}$ & $\begin{array}{l}\mathrm{p}- \\
\text { value }\end{array}$ & \multicolumn{4}{|c|}{ Model adjusted for: } \\
\hline $\begin{array}{l}\text { Total energy costs per } \\
\text { household }\end{array}$ & $\$ 76.65$ & 0.19 & \multicolumn{4}{|c|}{ Previous year, low income } \\
\hline $\begin{array}{l}\mathrm{CO}_{2} \text { emissions per household } \\
\text { (for total energy use) }\end{array}$ & $\$ 2.66$ & 0.18 & \multicolumn{4}{|c|}{ Previous year, low income, household in Dunedin } \\
\hline $\begin{array}{l}\text { Electricity costs per } \\
\text { household }\end{array}$ & $\$ 29.29$ & 0.53 & \multicolumn{4}{|c|}{ Previous year, household in Dunedin or Christchurch } \\
\hline $\begin{array}{l}\mathrm{CO}_{2} \text { emissions per household } \\
\text { (electricity use only) }\end{array}$ & $\$ 1.01$ & 0.53 & \multicolumn{4}{|c|}{ Previous year, household in Dunedin or Christchurch } \\
\hline & & & \multicolumn{2}{|c|}{$\begin{array}{l}\text { Total Dnergy Savings } \\
\left(\text { cost and } \mathrm{CO}_{2}\right)\end{array}$} & \multicolumn{2}{|c|}{$\begin{array}{l}\text { Total Blectricity Use } \\
\text { Savings (cost and } \mathrm{CO}_{2} \text { ) }\end{array}$} \\
\hline & $p$ - value & $\begin{array}{l}\text { Discount } \\
\text { rate }\end{array}$ & $\begin{array}{l}\text { Total over } \\
10 \text { year } \\
\text { horizon }\end{array}$ & $\begin{array}{l}\text { Total over } \\
20 \text { year } \\
\text { horizon }\end{array}$ & $\begin{array}{l}\text { Total over } \\
10 \text { year } \\
\text { horizon }\end{array}$ & $\begin{array}{c}\text { Total } \\
\text { over a } 20 \\
\text { year } \\
\text { horizon }\end{array}$ \\
\hline \multirow{9}{*}{$\begin{array}{l}\text { NPV of combined cost and } \\
\mathrm{CO}_{2} \text { savings per household } \\
\text { (All models) }\end{array}$} & $\mathrm{p}<0.05$ & $\mathrm{r}=0.025$ & $\$ 0.00$ & $\$ 0.00$ & $\$ 0.00$ & $\$ 0.00$ \\
\hline & & $\mathrm{r}=0.05$ & $\$ 0.00$ & $\$ 0.00$ & $\$ 0.00$ & $\$ 0.00$ \\
\hline & & $\mathrm{r}=0.10$ & $\$ 0.00$ & $\$ 0.00$ & $\$ 0.00$ & $\$ 0.00$ \\
\hline & $\mathrm{p}<0.10$ & $\mathrm{r}=0.025$ & $\$ 0.00$ & $\$ 0.00$ & $\$ 0.00$ & $\$ 0.00$ \\
\hline & & $\mathrm{r}=0.05$ & $\$ 0.00$ & $\$ 0.00$ & $\$ 0.00$ & $\$ 0.00$ \\
\hline & & $\mathrm{r}=0.10$ & $\$ 0.00$ & $\$ 0.00$ & $\$ 0.00$ & $\$ 0.00$ \\
\hline & $p<0.20$ & $\mathrm{r}=0.025$ & $\$ 694.18$ & $\$ 1,236.47$ & $\$ 0.00$ & $\$ 0.00$ \\
\hline & & $\mathrm{r}=0.05$ & $\$ 612.46$ & $\$ 988.45$ & $\$ 0.00$ & $\$ 0.00$ \\
\hline & & $\mathrm{r}=0.10$ & $\$ 487.36$ & $\$ 675.26$ & $\$ 0.00$ & $\$ 0.00$ \\
\hline
\end{tabular}

In general the effect of intervention was positive for both total energy use and electricity use, but the probability that this positive impact was the result of chance is relatively high, particularly with regard to electricity use. The difference between the probability outcome for total energy use and for electricity use is the result of including other energy costs in the analysis, particularly LPG, and indicates the importance of the decision to base the final cost benefit analysis on total energy use, rather than electricity use alone.

\subsection{Comfort}

Any increases in comfort resulting from the intervention are a calculated based on the total energy use savings reported above based on the assumption of a 30\% take-back (or 'comfort') effect. Changes in comfort are presented in table 17. 
Table 17: The Value of Changes in Comfort per Household as a Result of the Intervention

\begin{tabular}{|c|c|c|c|c|}
\hline \multirow{11}{*}{$\begin{array}{l}\text { NPV of changes in } \\
\text { comfort per household } \\
\text { for all models }\end{array}$} & & & \multicolumn{2}{|c|}{ Total Dnergy Use } \\
\hline & & & $10 \mathrm{yr}$ horizon & $\begin{array}{c}20 \mathrm{yr} \\
\text { horizon }\end{array}$ \\
\hline & $p<0.05$ & $r=0.025$ & $\$ 0.00$ & $\$ 0.00$ \\
\hline & & $r=0.05$ & $\$ 0.00$ & $\$ 0.00$ \\
\hline & & $r=0.10$ & $\$ 0.00$ & $\$ 0.00$ \\
\hline & $p<0.10$ & $\mathrm{r}=0.025$ & $\$ 0.00$ & $\$ 0.00$ \\
\hline & & $\mathrm{r}=0.05$ & $\$ 0.00$ & $\$ 0.00$ \\
\hline & & $r=0.10$ & $\$ 0.00$ & $\$ 0.00$ \\
\hline & $\mathrm{p}<0.20$ & $\mathrm{r}=0.025$ & 287.51 & 512.10 \\
\hline & & $r=0.05$ & 253.66 & 409.38 \\
\hline & & $\mathrm{r}=0.10$ & 201.85 & 279.67 \\
\hline
\end{tabular}

It is important to point out that, as discussed in Section 3.4.1, the method used to calculate the results in Table 17 has a notable flaw; it cannot estimate comfort benefits in the absence of a reduction in energy use. This means it cannot capture the comfort benefit of the increased living room and bedroom temperatures resulting from the intervention reported by Howden-Chapman et al. (2008, accepted with revisions) at p $<0.05$ and $\mathrm{p}<0.10$ levels, despite the fact that we can be reasonably certain that an improvement in comfort has occurred. This point is further discussed in Chapter 5 below.

\subsection{Care-giver days off work}

Following the calculation presented in Section 3.4.4 it is possible to estimate the additional benefits of the intervention in terms of care-giver days off work. This is set out in Table 18.

The results suggest a much greater positive outcome per household for Model B households, relative to Model A households and Model C households, reflecting the relative numbers of asthmatic children per household in the different models. Because changes in asthmatic children's days off school were significant at a $\mathrm{p}<0.05$ level and there were no statistically significant changes in non-asthmatic children's days off school, NPV calculations are only presented at a $\mathrm{p}<0.05$ level as they are identical for lower levels of statistical significance. 
Table 18: Benefit per Household of Reduction in Care-giver Days off Work due to Child's Illness

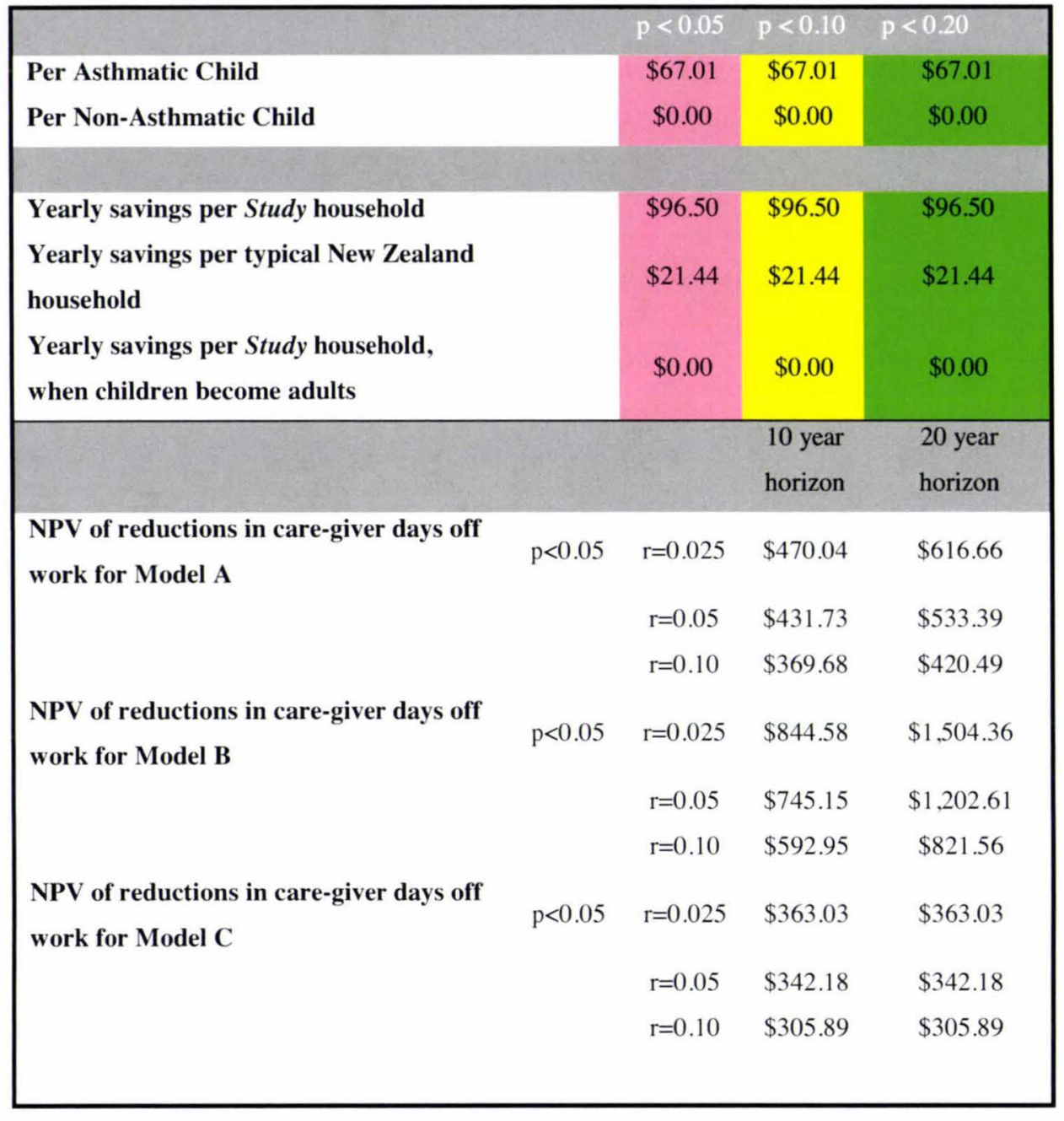

\subsection{Overall Cost Benefit Analysis}

This section sets out the results of the cost benefit analysis in Tables 18 and 19 .

Following the discussion in Chapter 3, the baseline scenario is Model A over a 20 year horizon with a p-value criterion of $\mathrm{p}<0.10$, a discount rate of 0.05 , and an assumption of four-yearly maintenance. The baseline scenario is highlighted in Table 20.

Initial analysis of the baseline scenario suggests that the intervention has a negative outcome from a cost benefit perspective, with a NPV per household of $-\$ 1,617.65$ and 
a benefit: cost ratio of $0.29: 1$. The choice of Model A,B or C does not significantly alter the basic outcome of the analysis, keeping all other variables constant; Model B resulted in a NPV of $-\$ 532.16$ and Model C resulted in a NPV of $-\$ 2,539.37$.

Discount rate does not have a major impact on the analysis of the baseline scenario, which reflects the fact that for Model A the majority of the health benefits per household occurred earlier in the period of analysis (e.g. before households with average population rates of asthma moved in). Analysis over a 10 year horizon also does not greatly alter the NPV of the intervention for the same reason.

The choice of statistical significance criterion has a noteworthy impact on the analysis. At p $<0.05$, the NPV of the intervention for the baseline scenario is $\$ 1,220.35$ and at $p<0.20$ it is $-\$ 11.25$; a major change. This reflects the fact that total energy use related savings and related comfort benefits are only included in the analysis at a $\mathrm{p}<$ 0.20 level. At a $\mathrm{p}<0.20$ there is a considerable positive benefit for Model B $(\$ 1,280.11)$ reversing the negative outcome for the same model at a $\mathrm{p}<0.10$ level.

The only 10 year horizon scenario which results in a positive NPV is Model B, p < $0.20, r=0.025(\$ 313.30)$.

In general it is difficult to draw any strong conclusions from the initial analysis. Although the baseline scenario clearly has a negative NPV, when energy use savings and comfort related savings are included at a $p<0.20$ level the NPV is nearly zero (generally considered a positive outcome from a cost benefit perspective). Several Model B scenarios result in a positive NPV. In this context it is clearly valuable to explore alternative scenarios and to consider issues beyond the direct scope of the analysis. 
Table 19: NPV of the Intervention per Household over a 10 Year Horizon

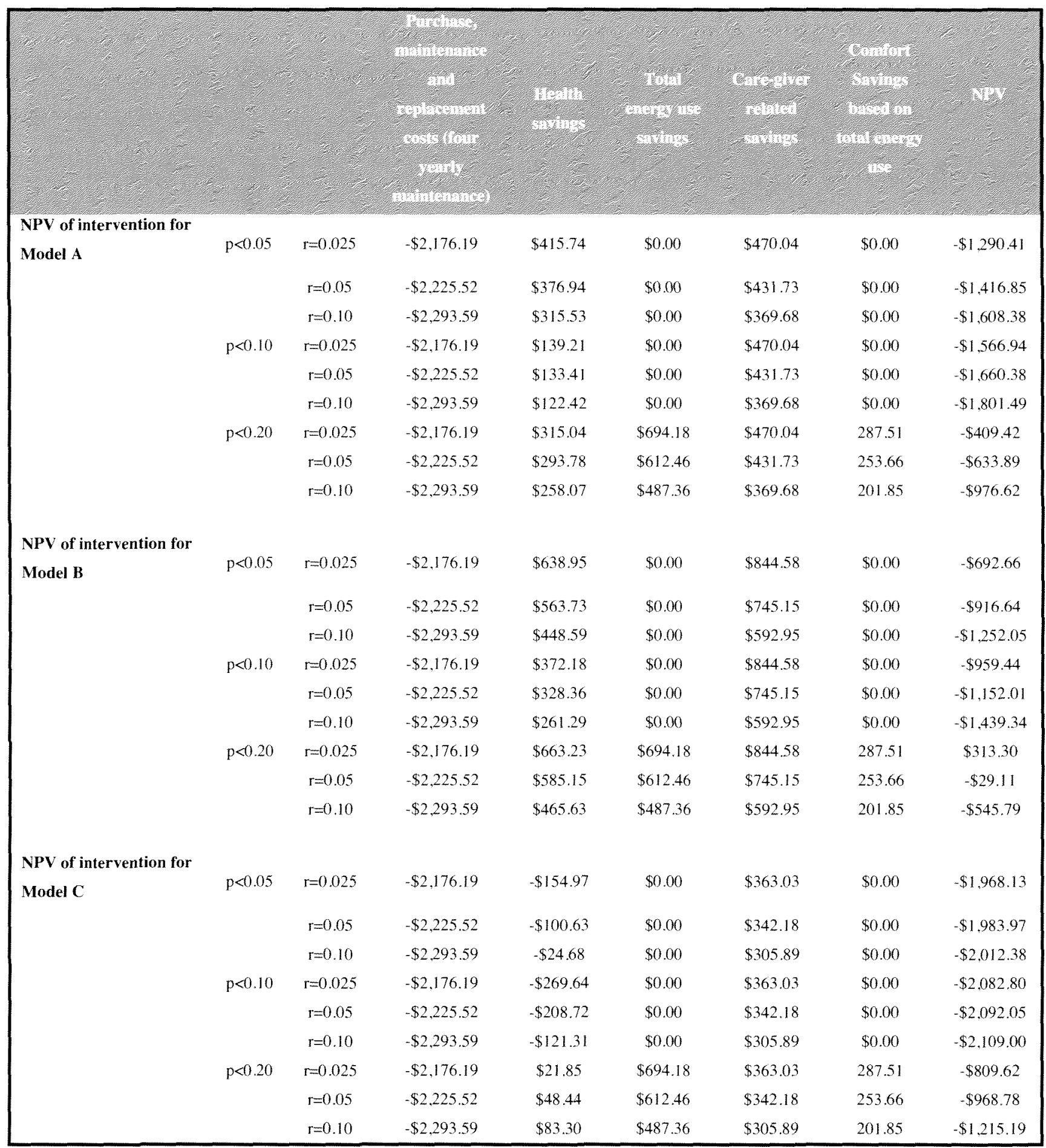


Table 20: NPV of the Intervention per Household over a 20 Year Horizon

\begin{tabular}{|c|c|c|c|c|c|c|c|c|}
\hline & & & $\begin{array}{l}\text { Purchase, } \\
\text { maintenance } \\
\text { and } \\
\text { replacement } \\
\text { costs (four } \\
\text { yearly } \\
\text { maintenance) }\end{array}$ & $\begin{array}{l}\text { Health } \\
\text { savings }\end{array}$ & $\begin{array}{l}\text { Total energy } \\
\text { use savings }\end{array}$ & $\begin{array}{l}\text { Care-giver } \\
\text { related } \\
\text { savings }\end{array}$ & $\begin{array}{c}\text { Comfort } \\
\text { Savings } \\
\text { based on } \\
\text { total energy } \\
\text { use }\end{array}$ & NPV \\
\hline \multirow{9}{*}{$\begin{array}{l}\text { NPV of } \\
\text { intervention for } \\
\text { Model A }\end{array}$} & $\mathrm{p}<0.05$ & $\mathrm{r}=0.025$ & $-\$ 2,234.88$ & $\$ 609.05$ & $\$ 0.00$ & $\$ 616.66$ & $\$ 0.00$ & $-\$ 1,009.18$ \\
\hline & & $\mathrm{r}=0.05$ & $-\$ 2,264.72$ & $\$ 510.97$ & $\$ 0.00$ & $\$ 533.39$ & $\$ 0.00$ & $-\$ 1,220.35$ \\
\hline & & $\mathrm{r}=0.10$ & $-\$ 2,312.46$ & $\$ 382.51$ & $\$ 0.00$ & $\$ 420.49$ & $\$ 0.00$ & $-\$ 1,509.47$ \\
\hline & $\mathrm{p}<0.10$ & $\mathrm{r}=0.025$ & $-\$ 2,234.88$ & $\$ 110.75$ & $\$ 0.00$ & $\$ 616.66$ & $\$ 0.00$ & $-\$ 1,507.48$ \\
\hline & & $\mathrm{r}=0.05$ & $-\$ 2,264.72$ & $\$ 113.68$ & $\$ 0.00$ & $\$ 533.39$ & $\$ 0.00$ & $-\$ 1,617.65$ \\
\hline & & $\mathrm{r}=0.10$ & $-\$ 2,312.46$ & $\$ 112.56$ & $\$ 0.00$ & $\$ 420.49$ & $\$ 0.00$ & $-\$ 1,779.42$ \\
\hline & $\mathrm{p}<0.20$ & $\mathrm{r}=0.025$ & $-\$ 2,234.88$ & $\$ 356.09$ & $\$ 1,236.47$ & $\$ 616.66$ & 512.10 & $\$ 486.44$ \\
\hline & & $\mathrm{r}=0.05$ & $-\$ 2,264.72$ & $\$ 322.24$ & $\$ 988.45$ & $\$ 533.39$ & 409.38 & $-\$ 11.25$ \\
\hline & & $\mathrm{r}=0.10$ & $-\$ 2,312.46$ & $\$ 272.29$ & $\$ 675.26$ & $\$ 420.49$ & 279.67 & $-\$ 664.75$ \\
\hline \multirow{9}{*}{$\begin{array}{l}\text { NPV of } \\
\text { intervention for } \\
\text { Model B }\end{array}$} & $\mathrm{p}<0.05$ & $\mathrm{r}=0.025$ & $-\$ 2,234.88$ & $\$ 1,138.10$ & $\$ 0.00$ & $\$ 1,504.36$ & $\$ 0.00$ & $\$ 407.57$ \\
\hline & & $\mathrm{r}=0.05$ & $-\$ 2,264.72$ & $\$ 909.81$ & $\$ 0.00$ & $\$ 1,202.61$ & $\$ 0.00$ & $-\$ 152.30$ \\
\hline & & $\mathrm{r}=0.10$ & $-\$ 2,312.46$ & $\$ 621.54$ & $\$ 0.00$ & $\$ 821.56$ & $\$ 0.00$ & $-\$ 869.36$ \\
\hline & $\mathrm{p}<0.10$ & $\mathrm{r}=0.025$ & $-\$ 2,234.88$ & $\$ 662.92$ & $\$ 0.00$ & $\$ 1,504.36$ & $\$ 0.00$ & $-\$ 67.61$ \\
\hline & & $\mathrm{r}=0.05$ & $-\$ 2,264.72$ & $\$ 529.95$ & $\$ 0.00$ & $\$ 1,202.61$ & $\$ 0.00$ & $-\$ 532.16$ \\
\hline & & $\mathrm{r}=0.10$ & $-\$ 2,312.46$ & $\$ 362.03$ & $\$ 0.00$ & $\$ 821.56$ & $\$ 0.00$ & $-\$ 1,128.86$ \\
\hline & $\mathrm{p}<0.20$ & $\mathrm{r}=0.025$ & $-\$ 2,234.88$ & $\$ 1,181.34$ & $\$ 1,236.47$ & $\$ 1,504.36$ & 512.10 & $\$ 2,199.38$ \\
\hline & & $\mathrm{r}=0.05$ & $-\$ 2,264.72$ & $\$ 944.38$ & $\$ 988.45$ & $\$ 1,202.61$ & 409.38 & $\$ 1,280.11$ \\
\hline & & $\mathrm{r}=0.10$ & $-\$ 2,312.46$ & $\$ 645.15$ & $\$ 675.26$ & $\$ 821.56$ & 279.67 & $\$ 109.19$ \\
\hline \multirow{10}{*}{$\begin{array}{l}\text { NPV of } \\
\text { intervention for } \\
\text { Model C }\end{array}$} & $\mathrm{p}<0.05$ & $\mathrm{r}=0.025$ & $-\$ 2,234.88$ & $-\$ 743.60$ & $\$ 0.00$ & $\$ 363.03$ & $\$ 0.00$ & $-\$ 2,615.45$ \\
\hline & & $\mathrm{r}=0.05$ & $-\$ 2,264.72$ & $-\$ 508.76$ & $\$ 0.00$ & $\$ 342.18$ & $\$ 0.00$ & $-\$ 2,431.29$ \\
\hline & & $\mathrm{r}=0.10$ & $-\$ 2,312.46$ & $-\$ 228.64$ & $\$ 0.00$ & $\$ 305.89$ & $\$ 0.00$ & $-\$ 2,235.20$ \\
\hline & $\mathrm{p}<0.10$ & $\mathrm{r}=0.025$ & $-\$ 2,234.88$ & $-\$ 858.27$ & $\$ 0.00$ & $\$ 363.03$ & $\$ 0.00$ & $-\$ 2,730.12$ \\
\hline & & $\mathrm{r}=0.05$ & $-\$ 2,264.72$ & $-\$ 616.84$ & $\$ 0.00$ & $\$ 342.18$ & $\$ 0.00$ & $-\$ 2,539.37$ \\
\hline & & $\mathrm{r}=0.10$ & $-\$ 2,312.46$ & $-\$ 325.26$ & $\$ 0.00$ & $\$ 305.89$ & $\$ 0.00$ & $-\$ 2,331.83$ \\
\hline & $\mathrm{p}<0.20$ & $\mathrm{r}=0.025$ & $-\$ 2,234.88$ & $-\$ 338.80$ & $\$ 1,236.47$ & $\$ 363.03$ & 512.10 & $-\$ 462.08$ \\
\hline & & $\mathrm{r}=0.05$ & $-\$ 2,264.72$ & $-\$ 201.62$ & $\$ 988.45$ & $\$ 342.18$ & 409.38 & $-\$ 726.32$ \\
\hline & & $\mathrm{r}=0.10$ & $-\$ 2,312.46$ & $-\$ 41.67$ & $\$ 675.26$ & $\$ 305.89$ & 279.67 & $-\$ 1,093.30$ \\
\hline & $\mathrm{p}<0.05$ & $\mathrm{r}=0.025$ & $-\$ 2,234.88$ & $\$ 609.05$ & $\$ 0.00$ & $\$ 616.66$ & $\$ 0.00$ & $-\$ 1,009.18$ \\
\hline
\end{tabular}




\section{DISCUSSION AND RECOMMENDATIONS}

The complexity of the cost benefit analysis, and the number of assumptions that it is based on mean that it is important to treat the results described in the previous chapter with caution. It is important to consider alternative scenarios and to place the analysis in the broader research and policy context.

\subsection{Alternative Scenarios}

Consideration of alternative scenarios is a useful way to explore the limitations of the data and the model.

\subsubsection{Maintenance}

One reasonably plausible combination of assumptions is a situation in which the Study heaters are not maintained professionally at all and thus perhaps only remain functional over a 10 year horizon. This can be considered a reasonably likely scenario, and it is interesting to analyse the results for that reason. It is, however, important to acknowledge that any decline in heater efficiency (with associated reductions in energy cost savings and health benefits) cannot be captured by this scenario. This scenario produces interesting results, with a negative NPV for all Model A scenarios, but a positive NPV for Model B over 10 years with a statistical significance criterion of $p<0.20$, and a discount rate of 0.05 or less (highlighted). This is particularly interesting because Model B is more plausible given a shorter horizon. Table 21 sets out the results of the non-maintenance scenario: 
Table 21: NPV of the Intervention per Household over a 10 Year Period with no maintenance

\begin{tabular}{|c|c|c|c|c|c|c|c|c|}
\hline & & & $\begin{array}{l}\text { Purchase, } \\
\text { maintenance } \\
\text { and } \\
\text { replacement } \\
\text { costs (non- } \\
\text { maintenance) }\end{array}$ & $\begin{array}{l}\text { Health } \\
\text { savings }\end{array}$ & $\begin{array}{l}\text { Total } \\
\text { energy } \\
\text { use } \\
\text { savings }\end{array}$ & $\begin{array}{l}\text { Care-giver } \\
\text { related } \\
\text { savings }\end{array}$ & $\begin{array}{l}\text { Comfort } \\
\text { Savings } \\
\text { based on } \\
\text { total } \\
\text { energy use }\end{array}$ & NPV \\
\hline \multirow[t]{9}{*}{$\begin{array}{l}\text { NPV of intervention } \\
\text { for Model A }\end{array}$} & $\mathrm{p}<0.05$ & $\mathrm{r}=0.025$ & $-\$ 2,056.73$ & $\$ 415.74$ & $\$ 0.00$ & $\$ 470.04$ & $\$ 0.00$ & $-\$ 1,170.95$ \\
\hline & & $\mathrm{r}=0.05$ & $-\$ 2,124.73$ & $\$ 376.94$ & $\$ 0.00$ & $\$ 431.73$ & $\$ 0.00$ & $-\$ 1,316.06$ \\
\hline & & $\mathrm{r}=0.10$ & $-\$ 2,221.58$ & $\$ 315.53$ & $\$ 0.00$ & $\$ 369.68$ & $\$ 0.00$ & $-\$ 1,536.37$ \\
\hline & $\mathrm{p}<0.10$ & $\mathrm{r}=0.025$ & $-\$ 2,056.73$ & $\$ 139.21$ & $\$ 0.00$ & $\$ 470.04$ & $\$ 0.00$ & $-\$ 1,447.48$ \\
\hline & & $\mathrm{r}=0.05$ & $-\$ 2,124.73$ & $\$ 133.41$ & $\$ 0.00$ & $\$ 431.73$ & $\$ 0.00$ & $-\$ 1,559.59$ \\
\hline & & $\mathrm{r}=0.10$ & $-\$ 2,221.58$ & $\$ 122.42$ & $\$ 0.00$ & $\$ 369.68$ & $\$ 0.00$ & $-\$ 1,729.48$ \\
\hline & $\mathrm{p}<0.20$ & $\mathrm{r}=0.025$ & $-\$ 2,056.73$ & $\$ 315.04$ & $\$ 694.18$ & $\$ 470.04$ & $\$ 258.75$ & $-\$ 318.71$ \\
\hline & & $\mathrm{r}=0.05$ & $-\$ 2,124.73$ & $\$ 293.78$ & $\$ 612.46$ & $\$ 431.73$ & $\$ 228.29$ & $-\$ 558.47$ \\
\hline & & $\mathrm{r}=0.10$ & $-\$ 2,221.58$ & $\$ 258.07$ & $\$ 487.36$ & $\$ 369.68$ & $\$ 181.66$ & $-\$ 924.79$ \\
\hline \multirow[t]{9}{*}{$\begin{array}{l}\text { NPV of intervention } \\
\text { for Model B }\end{array}$} & $\mathrm{p}<0.05$ & $r=0.025$ & $-\$ 2,056.73$ & $\$ 638.95$ & $\$ 0.00$ & $\$ 844.58$ & $\$ 0.00$ & $-\$ 573.20$ \\
\hline & & $\mathrm{r}=0.05$ & $-\$ 2,124.73$ & $\$ 563.73$ & $\$ 0.00$ & $\$ 745.15$ & $\$ 0.00$ & $-\$ 815.85$ \\
\hline & & $\mathrm{r}=0.10$ & $-\$ 2,221.58$ & $\$ 448.59$ & $\$ 0.00$ & $\$ 592.95$ & $\$ 0.00$ & $-\$ 1,180.04$ \\
\hline & $\mathrm{p}<0.10$ & $r=0.025$ & $-\$ 2,056.73$ & $\$ 372.18$ & $\$ 0.00$ & $\$ 844.58$ & $\$ 0.00$ & $-\$ 839.98$ \\
\hline & & $\mathrm{r}=0.05$ & $-\$ 2,124.73$ & $\$ 328.36$ & $\$ 0.00$ & $\$ 745.15$ & $\$ 0.00$ & $-\$ 1,051.22$ \\
\hline & & $\mathrm{r}=0.10$ & $-\$ 2,221.58$ & $\$ 261.29$ & $\$ 0.00$ & $\$ 592.95$ & $\$ 0.00$ & $-\$ 1,367.33$ \\
\hline & $\mathrm{p}<0.20$ & $\mathrm{r}=0.025$ & $-\$ 2,056.73$ & $\$ 663.23$ & $\$ 694.18$ & $\$ 844.58$ & $\$ 258.75$ & $\$ 404.01$ \\
\hline & & $\mathrm{r}=0.05$ & $-\$ 2,124.73$ & $\$ 585.15$ & $\$ 612.46$ & $\$ 745.15$ & $\$ 228.29$ & $\$ 46.32$ \\
\hline & & $\mathrm{r}=0.10$ & $-\$ 2,221.58$ & $\$ 465.63$ & $\$ 487.36$ & $\$ 592.95$ & $\$ 181.66$ & $-\$ 493.96$ \\
\hline \multirow{9}{*}{$\begin{array}{l}\text { NPV of intervention } \\
\text { for Model C }\end{array}$} & $\mathrm{p}<0.05$ & $\mathrm{r}=0.025$ & $-\$ 2,056.73$ & $-\$ 154.97$ & $\$ 0.00$ & $\$ 363.03$ & $\$ 0.00$ & $-\$ 1,848.67$ \\
\hline & & $\mathrm{r}=0.05$ & $-\$ 2,124.73$ & $-\$ 100.63$ & $\$ 0.00$ & $\$ 342.18$ & $\$ 0.00$ & $-\$ 1,883.18$ \\
\hline & & $\mathrm{r}=0.10$ & $-\$ 2,221.58$ & $-\$ 24.68$ & $\$ 0.00$ & $\$ 305.89$ & $\$ 0.00$ & $-\$ 1,940.37$ \\
\hline & $\mathrm{p}<0.10$ & $\mathrm{r}=0.025$ & $-\$ 2,056.73$ & $-\$ 269.64$ & $\$ 0.00$ & $\$ 363.03$ & $\$ 0.00$ & $-\$ 1,963.34$ \\
\hline & & $\mathrm{r}=0.05$ & $-\$ 2,124.73$ & $-\$ 208.72$ & $\$ 0.00$ & $\$ 342.18$ & $\$ 0.00$ & $-\$ 1,991.27$ \\
\hline & & $\mathrm{r}=0.10$ & $-\$ 2,221.58$ & $-\$ 121.31$ & $\$ 0.00$ & $\$ 305.89$ & $\$ 0.00$ & $-\$ 2,036.99$ \\
\hline & $\mathrm{p}<0.20$ & $\mathrm{r}=0.025$ & $-\$ 2,056.73$ & $\$ 21.85$ & $\$ 694.18$ & $\$ 363.03$ & $\$ 258.75$ & $-\$ 718.91$ \\
\hline & & $\mathrm{r}=0.05$ & $-\$ 2,124.73$ & $\$ 48.44$ & $\$ 612.46$ & $\$ 342.18$ & $\$ 228.29$ & $-\$ 893.36$ \\
\hline & & $\mathrm{r}=0.10$ & $-\$ 2,221.58$ & $\$ 83.30$ & $\$ 487.36$ & $\$ 305.89$ & $\$ 181.66$ & $-\$ 1,163.36$ \\
\hline
\end{tabular}




\subsubsection{Heater Type}

A limitation of the current analysis is that intervention group outcomes are the result of a particular combination of Study heaters installed. If, for example, the vast majority of health benefits or energy savings occurred only in households with pellet burners, this would not be captured by the analysis. An analysis by heater type installed was considered, but the limitations in terms of sample size (particularly for pellet burners and flued gas heaters) and analysis time meant that this option was not worth pursuing. A related issue is the question of to what extent the change from unflued gas heaters (given their additional health risks) was responsible for the improvement in health outcomes. At the time of writing He Kainga Oranga researchers are beginning a sub-analysis focusing only on households who reported an unflued gas heater as their main heater in 2005, which will address this question.

If it is assumed that the benefits of the intervention are identical for each Study heater type installed it is possible to calculate the NPV of the intervention per household for each heater type. Table 22 details the results of such a calculation assuming Model A with four yearly maintenance, $\mathrm{p}<0.10$ and $\mathrm{r}=0.05$.

Table 22 suggests that it is important that the results of the cost benefit analysis be interpreted with caution given the difference between the NPV of installing a heat pump and the NPV of installing a pellet burner over is over $\$ 1,200$ in favour of the heat pump over a twenty year period. It is also important to acknowledge that without information regarding any difference in health or energy use impacts for different heater types this conclusion is of limited value. 


\begin{tabular}{|llllll|}
\hline & & & & \\
\hline
\end{tabular}

The subject of price leads to the potential importance of economies of scale and of trends in the price of domestic space heaters. The Study heaters were purchased and installed with a reasonable discount in some cases. If a government body were to fund the purchase and installation of heaters on a large scale, for example in HNZC homes, the potential to obtain even greater discounts due to the scale of the operation would increase the likelihood of a positive outcome from a cost benefit analysis perspective. Additional factors that might improve the outcome include any future declines in the real cost of energy efficient heaters.

In terms of the baseline scenario, a massive discount of greater than $\$ 1,616.65$ would be required in order for the NPV of the intervention to be positive. Such a discount is highly unlikely. However, if energy and comfort related benefits are included (at a $\mathrm{p}<$ 0.20 level of statistical significance) the outcome is less clear cut, as the NPV of the 
intervention is $\quad-\$ 11.25$ and clearly even a moderate additional discount in heater prices would result in the calculation of a positive NPV.

\subsubsection{Take-back (comfort) effect}

One variable that it is particularly interesting is the estimate of comfort benefits resulting from the intervention. These were calculated assuming a $30 \%$ take-back effect. As this effect is an unknown, it is valuable to explore the potential impact that varying the $30 \%$ assumption may have. Assuming a 20 year horizon with a variety of discount rates and a statistical significance criterion of $p<0.20$ it is possible to calculate the impact of the intervention assuming either a $10 \%$ take-back or a $50 \%$ take-back and the results are displayed in Table 23. It is clear that the take-back effect is an important variable; in the case of Model $\mathrm{A}, \mathrm{r}=0.05$, which is closest to the baseline scenario (highlighted), a 50\% take-back effect means that the NPV of the intervention is positive, while it is negative assuming $10 \%$ or $30 \%$ take-back effects.

With respect to the highlighted scenario, following the discussion in Section 5.1.2, over a 20 year horizon even a slight increase in take-back related comfort benefits would result in a positive NPV.

Although previous international research suggests that a $30 \%$ take-back effect is likely, this analysis suggests that further research of the extent of the effect in the New Zealand context would be valuable, and would shed light on the current analysis. 
Table 23: NPV of Intervention based on assumption of $10 \%$ and $50 \%$ take-back effects

\begin{tabular}{|c|c|c|c|c|c|}
\hline & & & $\begin{array}{l}\text { NPV of } \\
\text { intervention } \\
\text { assuming } \\
10 \% \text { talke- } \\
\text { back }\end{array}$ & $\begin{array}{l}\text { NPV of } \\
\text { intervention } \\
\text { assuming } 30 \% \\
\text { take-back }\end{array}$ & $\begin{array}{l}\text { NPV of } \\
\text { intervention } \\
\text { assuming } 50 \% \\
\text { take-back }\end{array}$ \\
\hline \multirow[t]{3}{*}{ NPV of intervention for Model A } & \multirow[t]{3}{*}{$\mathrm{p}<0.20$} & $\mathrm{r}=0.025$ & $\$ 107.15$ & $\$ 486.44$ & $\$ 1,169.24$ \\
\hline & & $r=0.05$ & $-\$ 314.45$ & $-\$ 11.25$ & $\$ 534.60$ \\
\hline & & $\mathrm{r}=0.10$ & $-\$ 871.88$ & $-\$ 664.75$ & $-\$ 291.85$ \\
\hline \multirow[t]{3}{*}{ NPV of intervention for Model B } & \multirow[t]{3}{*}{$\mathrm{p}<0.20$} & $\mathrm{r}=0.025$ & $\$ 1,820.10$ & $\$ 2,199.38$ & $\$ 2,882.19$ \\
\hline & & $r=0.05$ & $\$ 976.90$ & $\$ 1,280.11$ & $\$ 1,825.95$ \\
\hline & & $\mathrm{r}=0.10$ & $-\$ 97.95$ & $\$ 109.19$ & $\$ 482.08$ \\
\hline \multirow[t]{3}{*}{ NPV of intervention for Model C } & \multirow[t]{3}{*}{$\mathrm{p}<0.20$} & $\mathrm{r}=0.025$ & $-\$ 841.36$ & $-\$ 462.08$ & $\$ 220.73$ \\
\hline & & $\mathrm{r}=0.05$ & $-\$ 1,029.52$ & $-\$ 726.32$ & $-\$ 180.47$ \\
\hline & & $\mathrm{r}=0.10$ & $-\$ 1,300.44$ & $-\$ 1,093.30$ & $-\$ 720.41$ \\
\hline
\end{tabular}

\subsubsection{Limiting analysis to Study data only}

Limiting analysis of the benefits of the intervention to data directly derived from the Study (i.e. excluding estimated care-giver and comfort related benefits) results in a negative NPV for all Models, horizons, statistical significance criteria and discount rates. Given that the goal of cost benefit analysis is to capture as many of the benefits of a proposed course of action as possible, this approach is unduly conservative.

\subsubsection{Energy costs}

The Ministry of Economic Development's report New Zealand's Energy Outlook to 2030 (MED, 2006) suggests that the real cost of residential energy will rise during the next twenty years, driven by the increasing price of natural gas and the costs associated with increasing generation capacity. If the environmental costs of electricity generation are internalised to a greater degree, for example via a carbon charge, this will also raise real energy prices. It is difficult to incorporate such changes into the cost benefit analysis but the relatively low price elasticity of demand in the residential electricity use sector suggests that it is likely that heating behaviours will not change dramatically following an increase in real costs (EECA 2003; MED, 2006), although there may be a minor substitution effect (Buckett, 2007), so it seems 
reasonable to assume that the health related benefits of the intervention will not be significantly altered. If the real price of energy is increased and we ignore such complications it is possible to observe the effect on the cost benefit analysis: this effect is presented in Table 24 for a variety of real energy cost scenarios (note that $\mathrm{p}<$ 0.20 and maintenance is assumed to occur every four years). The value of improved comfort is assumed to be unchanged.

Table 24: NPV of Intervention based on increased energy costs.

\begin{tabular}{|c|c|c|c|c|c|}
\hline & & $\begin{array}{l}\text { NPV of } \\
\text { intervention }\end{array}$ & $\begin{array}{l}\text { NPV of } \\
\text { intervention } \\
\text { assuming a } 10 \% \\
\text { increase in real } \\
\text { energy costs }\end{array}$ & $\begin{array}{l}\text { NPV of } \\
\text { intervention } \\
\text { assuming a } \\
30 \% \text { increase } \\
\text { in real energy } \\
\text { costs }\end{array}$ & $\begin{array}{l}\text { NPV of intervention } \\
\text { assuming a } 50 \% \\
\text { increase in energy } \\
\text { real energy costs }\end{array}$ \\
\hline \multicolumn{6}{|c|}{10 Year Horizon } \\
\hline \multirow[t]{3}{*}{ Model A } & $\mathrm{r}=0.025$ & $-\$ 409.42$ & $-\$ 340.01$ & $-\$ 201.17$ & $-\$ 62.33$ \\
\hline & $\mathrm{r}=0.05$ & $-\$ 633.89$ & $-\$ 572.64$ & $-\$ 450.15$ & $-\$ 327.66$ \\
\hline & $\mathrm{r}=0.10$ & $-\$ 976.62$ & $-\$ 927.89$ & $-\$ 830.42$ & $-\$ 732.94$ \\
\hline \multirow[t]{3}{*}{ Model B } & $\mathrm{r}=0.025$ & $\$ 313.30$ & $\$ 382.71$ & $\$ 521.55$ & $\$ 660.39$ \\
\hline & $\mathrm{r}=0.05$ & $-\$ 29.11$ & $\$ 32.14$ & $\$ 154.63$ & $\$ 277.12$ \\
\hline & $\mathrm{r}=0.10$ & $-\$ 545.79$ & $-\$ 497.06$ & $-\$ 399.59$ & $-\$ 302.11$ \\
\hline \multirow[t]{3}{*}{ Model C } & $r=0.025$ & $-\$ 809.62$ & $-\$ 740.20$ & $-\$ 601.37$ & $-\$ 462.53$ \\
\hline & $\mathrm{r}=0.05$ & $-\$ 968.78$ & $-\$ 907.53$ & $-\$ 785.04$ & $-\$ 662.55$ \\
\hline & $\mathrm{r}=0.10$ & $-\$ 1,215.19$ & $-\$ 1,166.45$ & $-\$ 1,068.98$ & $-\$ 971.51$ \\
\hline \multicolumn{6}{|c|}{20 year Horizon } \\
\hline \multirow[t]{3}{*}{ Model A } & $r=0.025$ & $\$ 486.44$ & $\$ 610.09$ & $\$ 857.38$ & $\$ 1,104.67$ \\
\hline & $r=0.05$ & $-\$ 11.25$ & $\$ 87.60$ & $\$ 285.29$ & $\$ 482.98$ \\
\hline & $\mathrm{r}=0.10$ & $-\$ 664.75$ & $-\$ 597.22$ & $-\$ 462.17$ & $-\$ 327.11$ \\
\hline \multirow[t]{3}{*}{ Model B } & $\mathrm{r}=0.025$ & $\$ 2,199.38$ & $\$ 2,323.03$ & $\$ 2,570.33$ & $\$ 2,817.62$ \\
\hline & $\mathrm{r}=0.05$ & $\$ 1,280.11$ & $\$ 1,378.95$ & $\$ 1,576.64$ & $\$ 1,774.33$ \\
\hline & $r=0.10$ & $\$ 109.19$ & $\$ 176.71$ & $\$ 311.77$ & $\$ 446.82$ \\
\hline \multirow[t]{3}{*}{ Model C } & $r=0.025$ & $-\$ 462.08$ & $-\$ 338.43$ & $-\$ 91.14$ & $\$ 156.16$ \\
\hline & $\mathrm{r}=0.05$ & $-\$ 726.32$ & $-\$ 627.47$ & $-\$ 429.78$ & $-\$ 232.09$ \\
\hline & $\mathrm{r}=0.10$ & $-\$ 1,093.30$ & $-\$ 1,025.77$ & $-\$ 890.72$ & $-\$ 755.67$ \\
\hline
\end{tabular}

It is clear that, for Model A over a 20 year horizon with $r=0.05$ (highlighted), which is the closest approximation to the baseline scenario, even a small increase in real energy costs will produce a positive NPV, with greater increases producing a notably positive NPV. Analysis of these scenarios suggests that in a future where real energy 
costs are predicted to increase, it is likely that an intervention of this kind will have a positive NPV.

Based on the alternative scenarios discussed above, the likely outcome of the intervention is less clear, as even slight changes in a number of variables will produce a positive net present value for Model A if energy savings and comfort benefits are also included in the analysis (at a $\mathrm{p}<0.20$ level).

\subsection{Limitations of the Analysis}

There are a number of factors that limit the value of the analysis such as the simplistic assumptions behind Models A, B and C, reliance on self reported data, the uncertainties regarding calculation of maintenance related costs, the fact that the outcomes of the analysis were based on the comparison of the particular mixture of Study heaters installed and those used in the control group (future interventions might produce different outcomes if particular heater types have a strong impact on the analysis), the uncertainties regarding comfort and care-giver related calculations and the limited size of the Study. The limited statistical power of the Study with respect to capturing changes in energy use is also a key limitation, given the impact of including energy use savings and comfort benefits on the outcome of the analysis. These limitations have been addressed where possible.

It is also important to note that the Study data only includes the South Island and the bottom of the North Island. The results of the analysis cannot be assumed to apply to the country as a whole.

More generally, the analysis has a limited scope of applicability; results cannot necessarily be generalised beyond dwellings with basic insulation occupied by households who previously used either an unflued gas heater or a plug in electric heater as their primary source of heat and include an asthmatic child aged seven to twelve. However, a priori, these are conditions that favour a positive outcome, which may suggest that the effects of installing energy efficient heating in "better" circumstances, e.g. better heating or lower rates of childhood asthma, are likely to be less positive from a societal perspective. 
However, it is certainly possible that installing Study type heaters in homes that already have higher than average indoor temperatures as a result of possessing more effective heating and/or insulation (double glazing for example) might result in greater energy use savings than the current intervention and thus a positive NPV (although additional health related benefits would be unlikely to occur). The current cost benefit analysis cannot be used to assess such a possibility

It is also possible that in situations where there are additional complicating variables, such as serious problems caused by external emissions from domestic space heating, that an intervention which involves replacing inefficient open and enclosed fires with Study type heaters might have a positive NPV. Another limitation that needs to be considered is the possibility that households containing a child with severe asthma might obtain much greater health related benefits from the intervention and that for such households the intervention might result in a positive NPV. The current analysis does not provide evidence to support or negate such a conclusion.

As noted in Section 3.2, it is not possible for the current analysis to fully capture any COPD, ischaemic heart disease or stroke related benefits, due to the size of the Study, the lack of mortality data, and because there were insufficient older Study participants to carry out a separate analysis of the health outcomes of this group. It is thus not reasonable to generalise the outcomes of the current analysis beyond families with children aged seven to twelve. Model $\mathrm{C}$ attempts to do so, but cannot account for changes in the health outcomes of adult household members as they age. This means that the current analysis does not provide a strong basis for assessing the NPV of installing Study type heaters into homes occupied by older people.

A further limitation of the analysis is the exclusion of potential benefits such as asthma cases avoided, reduced local external emissions, reductions in QALYs lost to mental illness and reduced structural repair costs. It is possible that if these benefits could be captured that the baseline scenario for the intervention would have a positive NPV, and it is very likely that the NPV of the intervention would be positive if energy and comfort benefits (significant at a $p<0.20$ level) were also included in the analysis. A more subtle issue is the exclusion of summer heat pump air-conditioning; 
such heat pump use potentially generates both higher energy costs and associated externalities and health and welfare gains, and the net effect is unclear (although an a priori consumer surplus argument would be that the welfare gains exceed the higher energy costs incurred).

A final consideration is that the current analysis should not be generalised beyond the domestic housing context. Replacing an unflued gas heater with a heat pump in a primary school, for example, could well have a much larger positive outcome owing to the greater number of asthmatic children who will benefit from such an intervention.

\subsection{The Research and Policy Context}

It is important to place the cost benefit analysis in the context of the Housing, Insulation and Health Study. Given the additional benefits of insulation which include a longer life-span, the lack of a maintenance requirement, and the elimination of complicating human variables including fuel poverty and difficulties in operating heaters, it was already reasonably clear, following the cost benefit analyses of Chapman et al. (2007) and Lloyd, Bishop and Callau (2007), that the primary focus of government household energy efficiency policy should be the installation of basic insulation. The current analysis would have had to produce a ratio of benefits to costs of about $1.7: 1$ or better to provide a case for prioritising the funding of energy efficient heating in already insulated homes ahead of insulating uninsulated homes. As discussed in Chapter 2, initiatives such as EECA's EnergyWise Home Grants programme already support the installation of basic insulation measures in pre 1978 homes and $\mathrm{HNZC}$ is currently carrying out a major retrofit of its pre-1978 housing stock (Lloyd, et al. 2007; EECA, 2008). The outcome of this cost benefit analysis suggests that the funding of Study type heaters should probably not be a government priority without further research unless a strong weighting is placed on health outcomes or credence is given to the alternative scenarios discussed in Section 5.1 (such as rising real energy costs) and in Section 5.2. The analysis suggests that the current focus on insulating uninsulated homes is well advised. 
Because analysis by Lloyd et al. (2007) of the HNZC retrofit in Dunedin only demonstrates modest temperature gains and few if any energy savings, Lloyd, Bishop and Callau (2007), as discussed previously, explore a more extensive efficiency upgrade. The authors' analysis does not include any take-back effect and calculates a positive NPV for installing a pellet burner in the living room of a house with basic insulation, and subsequently a heat pump elsewhere in the house. The current analysis, while not directly comparable, does raise the possibility, which the authors acknowledge, that such calculations may be overly optimistic in the light of occupant behaviour; even including health, comfort and caregiver benefits, the current analysis leads to a much more equivocal conclusion.

EECA's voluntary HERS programme, which enables home-owners to obtain an energy rating, is intended to encourage energy efficiency improvements including improved domestic space heating. The cost benefit analysis does not provide strong evidence as to the benefit of encouraging investment in such heaters, given complicating factors such as international research suggesting that only an educated and wealthy minority are likely to make decisions based on HERS (Farhar, 2000). If a voluntary HERS encourages the installation of energy efficient heating into homes that already have higher than average temperatures (as a result of effective insulation, effective heating or high household incomes), it may be, as discussed above, that this will produce positive net social outcomes for New Zealand via energy savings: the current analysis does not preclude this possibility.

The cost benefit analysis also does not suggest that initiatives such as the Clean Heat Project may be misguided; these projects are distinctive as they involve replacing different types of heaters (open and enclosed fires) in areas with additional smog related health problems, and take place in a specific regulatory context. It may, however suggest that further economic analysis of such interventions would be of interest.

In general, the limitations of the Study data in terms of sample size and statistical power suggest that there is a need for research on heating interventions at a larger scale but, as discussed in Chapter 3, this may be unlikely given the high degree of cost involved and as evidenced by the lack of previous research. In the context of global 
warming and rising real energy costs, the payoff from more efficient and sustainable heating is likely to rise over time, and it may not be advisable to wait for such research to be carried out.

The current analysis also suggests a number of other potentially valuable areas for future research. One of the first areas to be explored should certainly be a cost benefit analysis that focuses on the subset of Study households who used an unflued gas heater in 2005; such an analysis will potentially eliminate some of the uncertainties that limit the current research. Lloyd, Bishop and Callau (2007) produce a hierarchy of energy efficiency improvements with ceiling and floor insulation the first priority; it would be valuable to carry out research similar to the He Kainga Oranga studies with regard to other energy efficiency improvements that the authors discuss such as double glazing and cavity insulation. Other areas for further exploration include quantitative and qualitative research regarding appliance maintenance behaviour, research regarding the mobility of owner-occupiers in New Zealand and the cost, in terms of time-off work for care-givers, of days off school for sick children.

It is important to acknowledge the fundamental limitations of cost benefit analysis, from a policy perspective, which include the false sense of concreteness produced and the existence of issues that are beyond the scope of the analysis. In the present case, such intangibles include the value that is placed on reducing the distress and suffering of asthmatic children. It is difficult to assess the cost of such suffering, but it is certainly possible that the reductions in wheezing and other symptoms reported in Howden-Chapman et al. (2008, accepted with revisions), might represent sufficient value for an individual household to consider purchasing a Study type heater, especially if the child in that household has a particularly serious condition.

Cost benefit analysis, as discussed in Section 3.1, does not engage with ethical issues. Relevant ethical concerns, from a governmental perspective, may include the issue of continuing to allow the purchase and use of unflued gas heaters which are likely to cause health problems for users. Such concerns may justify the promotion of Study type heaters as a safe alternative, even given an uncertain net social benefit from a national perspective. More generally, there are moral issues surrounding the continued exposure of children and adults to cold, damp and mouldy environments as a result of 
fuel poverty and inefficient heating. The moral calculus involved in valuing health benefits and in resolving these issues is beyond the scope of the current analysis and it is thus important to reiterate the point that cost benefit analysis is merely one of a number of tools that policy makers can use to assess complex problems.

One of the interesting points that can be made following the cost benefit analysis is that untargeted improvements in household energy efficiency may not be the most viable or effective way to improve the health of asthmatic children from a governmental perspective. This is a result of population mobility and aging. A reasonable approach from a government perspective might be to target housing that is more likely to be continuously occupied by children with higher rates of asthma, for example low income HNZC homes; this is roughly analogous with Model B.

However, following the current cost benefit analysis, it is uncertain whether such a targeted intervention will produce positive net social outcomes. It is also important to note that care-giver related savings were calculated using average female wage rates and average employment rates for mothers and therefore the benefits of targeting low income households in terms of care-giver savings may be lower when factoring in higher unemployment rates and lower wage rates. 


\section{CONCLUSION}

In conclusion, the cost benefit analysis of the Housing, Heating and Health Study indicates that the intervention did not result in a positive NPV for the baseline scenario. This suggests that governmental investment in, or funding of, Study type domestic space heating for households with asthmatic children that currently have insulation but poor heating may not necessarily produce net benefits for New Zealand society.

However, there are a number of inherent limitations with regard to the data used, assumptions made and the method adopted, and for a number of scenarios, where energy use savings and comfort are included in the analysis, the intervention is predicted to result in a positive NPV. Future research with the statistical power to assess changes in energy use more effectively and the ability to accurately quantify improvements in comfort and to capture potential benefits such as reductions in new cases of asthma and QALYs lost to mental illness might well indicate a generally positive NPV.

More generally, there are a number of changes facing the New Zealand economy with regard to climate change mitigation measures and rising energy costs which are likely to have a large impact on future domestic space heating costs and while it is not possible to fully assess the impact of these and other variables, it seems likely that if real energy costs rise an intervention of this type may well produce positive net social outcomes.

It is also important to note that cost benefit analysis cannot capture the ethical component of policy decisions regarding health outcomes and as such is inherently limited.

In this context, it is important to again emphasise the equivocal nature of the conclusion stated above. 


\section{REFERENCES}

Access Economics (2005). The Economic Cost of Arthritis in New Zealand. A report prepared by Access Economics Pty for Arthritis New Zealand.

Access Economics (2005B). Arthritis - the bottom line. The economic impact of arthritis in Australia. A report prepared by Access Economics for Arthritis New Zealand.

Aish, H., Didsbury, P., Cressey, P., Grigor, J., Gribben, B. (2003) Primary Options for Acute Care: general practitioners using their skills to manage "avoidable admission" patients in the community. The New Zealand Medical Journal. Vol. 1169.

Arrow, K.J., Cropper, M.L., Eads, G.C, Hahn, R.W., Lave, L.B, Noll, R.G., Portney, P.R., Russell, M., Schmalensee, R., Smith, V.K, Stavins, R.N. (1996). Benefit-Cost Analysis in Environmental, Health, and Safety Regulation. American Enterprise Institute.

Asher, M.I., Barry, D., Clayton, T., et al. (2001) The International Study of Asthma and Allergies in Childhood (ISAAC) Phase One. The burden of symptoms of asthma, allergic rhinoconjunctivitis and atopic eczema in children and adolescents in six New Zealand centers: ISAAC Phase One. NZ Med J, 114: $114-20$

Aylin, P., Morris, S., Wakefield, J., Grossinho, A., Jarup, L., Elliott, P.( 2001). Temperature, housing, deprivation and their relationship to excess winter mortality in Great Britain, 1986-1996.

International Journal of Epidemiology, 30:1 100-1108

Barck C, Sandstrom T, Lundahl J, Halldén G, Svartengren M, Strand V, et al. (2002). Ambient level of $\mathrm{NO}_{2}$ augments the inflammatory response to inhaled allergen in asthmatics. Respiratory Medicine, 96(11): $907-917$

Basagaña, X, Sunyer, J., Kogevinas, M., Zock, Z., Duran-Tauleria, E.,Jarvis D., Burney, P., Maria, J. on Behalf of the European Community Respiratory Health Survey (2004). Socioeconomic Status and Asthma Prevalence in Young Adults: The European Community Respiratory Health Survey. American Journal of Epidemiology. 160:178-188

Biddulph, F., Biddulph, J., Biddulph, C. (2003). The complexity of community and family influences on children's achievement in New Zealand: Best Evidence Synthesis. Report prepared for the New Zealand Ministry of Education.

Binswanger, M (2000). Technological progress and sustainable development: what about the rebound effect? Ecological Economics, 36: 119-132.

Boulic, M. Phipps, R. (2008). Is mould presence related to heating? - other moisture sources in the home. Presentation.

Buckett, N.R. (2007) National Impacts of the Widespread Adoption of Heat Pumps in New Zealand. BRANZ Study Report No. 169 (2007). Judgeford, New Zealand.

Bushnell, J. (2007). Psychological Medicine: Research. The MaGPIe Study: The Mental Health and General Practice Investigation. http://www.otago.ac.nz/wsmhs/academic/psych/research/magpie.html. Accessed 07/11/07.

Carrer, P., Maroni, M., Alcini, D., Cavallo, D.(2001). Allergens in indoor air: environmental assessment and health effects. The Science of the Total Environment, 270: 33-42.

Chapman, R. (2007) The incremental costs of health-related consequences of leaky dwellings: a preliminary economic estimate. Appendix 1. Wilson, N., Howden-Chapman, P., Crane, J., Wickens, K., Chapman, R. (2007). Potential Health Impacts Associated with Mould in "Leaky Buildings": A review commissioned by the Auckland City Council. 
Chapman R, Howden-Chapman P, O'Dea D, Viggers H, Kennedy M. (2007) Retrofitting houses with insulation: a cost-benefit analysis of a randomized community trial. In review. Wellington, New Zealand; Housing and Health Research Programme,

www.maarama.co.nz/Insulation_Benefits_feb07.doc. Accessed 05/06/07.

Chauhan, A.J., Inskip, H.M., Linkaker, C.H. et al. (2003). Personal exposure to nitrogen dioxide $\left./ \mathrm{NO}_{2}\right]$ and the severity of virus induced asthma in children. The Lancet, 361:1939-1944.

Clark S.J., Jones, M., Page I.C. (2005). New Zealand 2005 House Condition Survey. BRANZ Study Report 142. 2005. BRANZ Ltd, Judgeford, New Zealand.

Clinch, J.P., Healy, J.D. (2001). Cost-benefit analysis of domestic energy efficiency. Energy Policy, 29: 113-124.

Clark and Onaka (1983). Life Cycle and Housing Adjustment as Explanations of Residential Mobility. Urban Studies, 20: 47-57.

Cogan (1996). The New Zealand Building Code -Implications and Opportunities for Designers. http:/www.ema.org.nz/publications/papers/cogan 1996.pdf. Accessed 04/12/07.

Consumer (2007) $9 \mathrm{~kg}$ bottled LPG prices.

http:/www.consumer.org.nz/newsitem.asp?docid $=2851 \&$ category $=$ News $\&$ topic $=9 \mathrm{~kg} \% 20 \mathrm{bottled} \% 20 \mathrm{~L}$ PG\%20prices. Accessed 09/09/07.

Consumer Build (2008). Background to the problem.

http://www .consumerbuild .org.nz/publish/leaky/leaky-background.php. Accessed 03/03/08.

Consumer Build (2008A). Case Study 1. http://www.consumerbuild.org.nz/publish/leaky/leakycasestudy-1.php. Accessed 03/03/08.

Critchley, R., Gilbertson, J., Green, G., Grimsey, M. (2004). Housing investment and health in Liverpool. Sheffield: CRESR. Sheffield Hallam University.

Critchley, R., Gilbertson, G., Green, G. and the Warm Front Study Group. (2007). Living in Warm Homes after heating improvements: Evidence from Warm-Front, England's Home Energy Efficiency Scheme. Applied Energy, Vol. 84, 2: 147-158.

Daly, H.E., Farley, J., (2004). Ecological Economics: principles and applications. Island Press, Washington, D.C.

Davie, G.S., Baker, M.G., Hales, S., Carlin, J.B. (2007). Trends and determinants of excess winter mortality in New Zealand: 1980 to 2000. BMC Public Health, 7: 263.

DBH (2007). Building Code Review 2007 Consultation: Discussion Document. http://www.dbh.govt.nz/bcr-discussion-document-index. Accessed 05/01/08.

DEFRA (2005). The Government's Standard Assessment Procedure for Energy Rating of Dwellings. BRE, Garston, Watford. http://www.projects.bre.co.uk/sap2005/pdf/SAP2005.pdf. Accessed 06/09/07.

Denne, T. (2007) Environmental Costs of Electricity Generation. Environment Waikato Technical Series $2007 / 09$.

D'Souza, W., Lewis, S., Cheng, S., McMillan, D., Pearce, N., et al. (1999). The prevalence of asthma symptoms, bronchial hyperresponsiveness and atopy in New Zealand adults. New Zealand Medical Journal, 112: 198-202.

EECA (2003). Exploring our Untapped Electricity Resource.

http:/www.eecabusiness.govt.nz/emprove/emprove-library/implementation/demandresponse/report/demand-side-participation-in-the-nz-market.pdf Accessed 20/03/08. 
ECAN (2006). Clean Heat Project Incentives and assistance for clean heating and energy efficiency in the Christchurch area. http://www.ecan.govt.nz/NR/rdonlyres/528A33D9-42D0-4A56-88273B6134CEC30D/0/CleanHeatrevisedpolicy pdf Accessed 02/02/08.

ECAN (2007) Canterbury Natural Resources Regional Plan. Chapter 3. http:/www .ecan.govt.nz/NR/rdonlyres/AD 1B5C1E-5C2C-43BC-974E97A6F17B0D21/0/Ch3NewptlPgs 1 to58.pdf. Accessed 21/11/07.

EECA (2007) New Zealand Energy Efficiency and Conservation Strategy. Energy Efficiency and Conservation Authority. ISBN 978-0-478-19503-3 (PDF) http:/www.eeca.govt.nz/eeca-library/eecareports/neecs/report/nzeecs-07.pdf. Accessed 07/02/08

EECA (2008) Home Energy Rating Scheme. http:/www.eeca.govt.nz/residential/home-energy-ratingscheme/indexnew html accessed 02/03/08.

EECA (2008A) EnergyWise Home Grants. http://www .eeca.govt.nz/residential/energywise-homegrants/index.html. Accessed 21/02/08.

Evan, G.W., Wells, N.M., Moch, A. (2003). Housing and Mental Health: A Review of the Evidence and a Methodological and Conceptual Critique. Journal of Social Issues. Vol 59, 3: 475-500.

Evans, J., Hyndman, S., Stewart-Brown, S. et al. An epidemiological study of the relative importance of damp housing in relation to adult health. J Epidemiol Community Health, 54: 677-686.

Fabitz, R., Feinleib, M. (1980). Geographic patterns in country mortality rates from cardiovascular diseases. American Journal of Epidemiology, 111: 315-328.

Farhar, B.C., Collins, N.E. and Walsh, R.W. Linking home energy rating systems with energy efficiency financing: progress on national and state programs. NREL. NREL/TP-460-21322.

Fisk, W.J., Lei-Gomez, Q., Mendell, M.J. (2007). Meta-analyses of the associations of respiratory health effects with dampness and mold in homes. Indoor Air, 17: 284-296.

French, L.J., Camilleri, M.J., Isaacs, N.P., Pollard, A.R. (2007). Temperatures and heating energy in New Zealand houses from a nationally representative study-HEEP. Energy and Buildings

Vol.39, 7: 770-782

French, L.J. (2008). Active Cooling and Heat Pump use in New Zealand-Survey Results. BRANZ Study Report No. 186. http:/www.branz.co.nz/branzltd/publications/pdfs/SR186_Heat_Pumps.pdf. Accessed 04/04/08.

Fyfe, C. (2005). Heat Pump Survey - Report 2: Winter 2004. Community Energy Action Charitable Trust, Christchurch, New Zealand.

Gilbertson, J., Stevens, M., Stiell, B., Thorogood, N. (2006). Home is where the hearth is: Grant recipients' views of England's Home Energy Efficiency Scheme (Warm Front). Social Science and Medicine, 63: 946-956.

Goodacre, C., Sharples, S., Smith, P. (2002) Integrating energy efficiency with social agenda in sustainability. Energy and Buildings. 34: 53-61.

Hales, S., Salmond, C. Town, G.I., Kjelstrom, T., Woodward, A. (1999) Daily Mortality in relation to weather and air pollution in Christchurch, New Zealand. Australian and New Zealand Journal of Public Health. Vol. 24, 1,

Hansen, J., Sato, M., Kharecha,P.Beerling, D. Masson-Delmotte, V. Pagani, M. Raymo, M., Royer, D., Zachos, J.C. Target Atmospheric CO2: Where Should Humanity Aim? Columbia University. http://www.columbia.edu/ jehI/2008/TargetCO2_20080317.pdf. Accessed 25/03/08. 
Harrington, B.E., Heyman, B., Merleau-Ponty, N., Stockton, H., Ritchie, N., Heyman, A. (2005) Keeping warm and staying well: findings from the qualitative arm of the Warm Homes Project. Health and Social Care in the Community, Vol. 13, 3: 259-267.

He Kainga Oranga, Chapman, R., Westergard, D. (2005) Housing, Heating and Health Study: heater Analysis. University of Otago, Wellington School of Medicine and Health Sciences.

Heinzerling, L. and Ackerman, F. (2002) Pricing the Priceless: Cost-Benefit Analysis of Environmental Protection, 150 U. PENN. L. REV. 1553 (symposium issue)

Herring, H. (2006). Energy efficiency - a critical view. Energy, Vol. 31: 10-20.

Herring, H., Roy, R. (2002). Sustainable services, electronic education and the rebound effect. Environmental Impact Assessment Review, 22: 525-542.

HNZC (2005). Building the Future: The New Zealand Housing Strategy. Housing New Zealand Corporation. http://www.hnzc.co.nz/hnzc/web/research-\&-policy/strategy-publications/nzhs/onlineversion/online-version---new-zealand-housing-strategy.htm. Accessed 04/10/07.

Holt, S., Beasley, R. (2001) The Burden of Asthma in New Zealand. Wellington: Asthma and Respiratory Foundation of New Zealand (Inc.).

Hong, S.H., Oreszczyn, T., Ridley, I. and the Warm Front Study Group. The impact of energy efficient refurbishment on the space heating fuel consumption in English dwellings. Energy and Buildings. 38: $1171-1181$.

Hopton, J.L, Hunt, S.M. (1996) Housing Conditions and mental health in a disadvantaged area in Scotland. Journal of Epidemiology and Community Health. 50: 56-61.

Howden-Chapman, P. (2004). Housing Standards: a Glossary of housing and health. Journal of Epidemiology and Community Health. 58: 162-168.

Howden-Chapman, P., Crane, J., Matheson, A., Viggers, H., Cunningham, M., Blakely, T., et al. (2005). Retrofitting houses with insulation to reduce health inequalities: aims and methods of a clustered, randomised trial in community settings. Social Science and Medicine, 61, 2600-2610

Howden-Chapman, P., Saville-Smith, K., Crane, J., Wilson, N. (2005A) Risk factors for mould in housing: a national survey. Indoor Air. 15: 469-476.

Howden-Chapman, P., Matheson, M., Crane, J. Viggers, H., Cunningham, M., Blakely, T., Cunningham, C., Woodward, A. Saville-Smith, K., O'Dea, D., Kennedy, M., Baker, M., Waipara, N., Chapman, R., Davie, G. (2007).Effect of insulating existing houses on health inequality: cluster randomized study in the community. BMJ, doi:10.1136/bmj.39070.573032.80

Howden-Chapman, P., Pierse, N., Nicholls, S., Gillespie-Bennett, J., Cunningham, M., Phipps, R., Boulic, M., Fjällström, P., Free, S., Chapman, R., Lloyd, B., Viggers, H., Wickens, K., Shields, D., Baker, M., Cunningham, C., Woodward, A., Bullen, C., Crane, J. (2008, accepted with revisions). Reducing childhood asthma morbidity through a randomised housing intervention: main health results from the Housing, Heating and Health Study. BMJ.

Hunt, S. (1990). Emotional distress and bad housing. Health and Hygiene. 11: 72-79.

IOM (2004). Damp indoor spaces and health. Institute of Medicine, Washington D.C. National Academy Press.

IPCC (2007). Climate Change 2007: Mitigation. Contribution of Working Group III to the Fourth Assessment Report of the Intergovernmental Panel on Climate Change (Metz, B., Davidson, O.R., Bosch, P.R., Dave, R.,Meyer, L.A. (eds.)), Cambridge University Press, Cambridge, United Kingdom and New York, NY, USA. 
Isaacs, N.P, Camilleri, M., French, L., Pollard, A., Saville-Smith, K., Fraser, R., Possouw, P. Jowett, J. (2006) Energy Use in New Zealand Households: Report on the Year 10 Analysis for the Household Energy End-use Project (HEEP). Branz Ltd. Study Report 155, Judgeford, New Zealand.

Isaacs, N.P., Camilleri, M., French, L., Pollard, A., Saville-Smith, K., Fraser, R., Rossouw, P. (2005). Energy Use in New Zealand Households: Report on the Year 9 Analysis for the Household Energy End-use Project (HEEP). Branz Ltd. Study Report 141, Judgeford, Porirua.

Iverson, M., Bach, E., Lundqvist, G.R. (1986). Health and comfort changes among tenants after retrofiting of their housing.

Environment International, Vol. 12: 161-166.

Jakob, A., Craig, J.L., Fisher, G., (2006). Transport cost analysis: a case study of the total costs of private and public transport in Auckland. Environmental Science and Policy, Vol. 9: 55-66.

Johnston, G. (2005) Women's participation in the labour force. New Zealand Treasury. Working Paper 05/06.

Keatinge, W.R., Donaldson, G.C. and the Eurowinter Group Study Organisation (1997). Cold exposure and winter mortality from ischaemic heart disease, cerebrovascular disease, respiratory disease, and all causes in warm and cold regions of Europe. The Lancet. Vol. 349: 1341-1346.

Kercsmar, C.M., Dearborn, D.G., Schluchter, M., Xue, L., Kirchner, H.L., Sobolewski, J., Greenberg, S.J., Vesper, S.J., Allan, T. (2006) Reduction in Asthma Morbidity in Children as a Result of Home Remediation Aimed at Moisture Sources. Environmental Health Perspectives, Vol. 114, 8: 1574-1580.

Kerstjens, H.A., Postma, D.S. (2002) Chronic obstructive pulmonary disease. American Family Physician, 66(5):845-6.

Laforest, L.,; Yin, D., Kocevar, V.S., Pacheco, Y., Dickson, N. Gormand, F. van Ganse, E. (2004). Association between asthma control in children and loss of workdays by caregivers. Annals of Allergy, Asthma and Immunology. Vol 93, 3: 265-271

Layard, R., Clark, D., Knapp, M., Mayraz, G. (2006). Implementing the NICE guidelines for depression and anxiety. A cost-benefit analysis. Preliminary draft. Available:

http://cep.Ise.ac.uk/textonly/research/mentalhealth/RL446g.pdf. Accessed 05/12/07.

Lloyd, B. (2006). Fuel Poverty in New Zealand. Social Policy Journal of New Zealand. 27: 142-155.

Lloyd, B., Bishop, T., Callau, M. (2007). Retrofit alternatives for State Houses. Report No. 2. Energy Management Group, Physics Department - University of Otago - Dunedin - New Zealand

Lloyd, C.R., Callau, M.F., Bishop, T., Smith, I.J. (2007) The Efficacy of an Energy Efficient Upgrade Program in New Zealand. doi:10.1016/j.enbuild.2007.11.006

Marno, P., Bryden, C., Bird, W., Watkin, H.A. (2005). How different measures of cold weather affect chronic obstructive pulmonary disease (COPD) hospital admissions in London. European Respiratory Review. Vol. 15, No. 101: 185-186.

Masoli, M., Fabian, D., Holt, S., Beasley, R. The global burden of asthma: executive summary of the GINA

Dissemination Committee Report. Allergy 2004: 59: 469-478.

McNaughton, S., Smith, L., Rea, H., Asher, I., Mitchell, E. et al. (1993). The Management of Childhood Asthma: attendance and school performance. New Zealand Journal of Educational Studies, 28(2), 155

MED (2006). Schedule of Domestic Natural Gas Prices Available up to 1 March 2006. http://www.med.govt.nz/templates/MultipageDocumentTOC 19797.aspx. Accessed 05/08/07. 
Melia RJW, Florey CV, Morris R, Goldstein BD, Clark D, \& John H. (1982). Childhood respiratory illnesses and the home environment: relations between nitrogen dioxide temperature and relative humidity. Int J Epidemiol, 11, 155-163.

Milne, G. and Boardman, B. Making Cold Homes Warmer: the effect of energy efficiency improvements in low-income homes. Energy Policy Vol. 28: 411-423

MED. Energy Data File. www med.govt.nz.

MED (2006). (Samuelson, R., Wilkinson, D., Walkington, M., Fairclough, R., Wilkinson, B., Hammond, K., Armstrong, T., Tsui, K.). New Zealand's Energy Outlook to 2030. http://www.med.govt.nz/upload/38641/eo-2006-final.pdf. Accessed 07/07/07.

MED (2007) (Samuelson, R., Wilkinson, D., Lawrence, S., Hammond, K., Walkington, M., Pickup, M.). Benefit-Cost Analysis of the New Zealand Energy Strategy. Ministry of Economic Development.

MED (2007A). New Zealand Energy Greenhouse Gas Emissions 1990-2006. Ministry of Economic Development, Wellington. ttp://www.med.govt.nz/upload/49905/1_GHG_report.pdf. Accessed 03/11/07.

MfE (2004). Warm Homes Technical Report: Social Drivers: Phase 1: Interim Progress Report. http://www.mfe.govt.nz/publications/energy/warm-homes-social-drivers-phase 1nov05/html/index.html. Accessed 05/05/07.

MfE (2005). Resource Management (National Environmental Standards Relating to Certain Air Pollutants, Dioxins, and Other Toxics) Regulations 2004.

http://www .mfe.govt.nz/laws/standards/consolidated-nes-aug2005.pdf

MfE (2005) Warm Homes Technical Report: Detailed Study of Heating Options in New Zealand. Phase I Report. ME number: 699.

MfE (2007). Projected Balance of Emissions Units During the First Commitment Period of the Kyoto Protocol. Ref. ME 808. http:/www.mfe.govt.nz/publications/climate/projected-balance-emissionssep07/index.html. Accessed 18/11/07.

MfE (2007A) New Zealand's Greenhouse Gas Emissions.

http:/www.mfe.govt.nz/publications/climate/nir-jul07/nir-jul07.pdf. Accessed 08/07/07.

MOH (2005). Unflued Gas Heaters and Your Health.

http://www.moh.govt.nz/moh.nsf/indexmh/unflued-gas-heaters-may05. Accessed 04/04/07.

Mudarri, D., Fisk, W.J. (2007) Public health and Economic Impact of Dampness and Mold. Indoor Air 17: 226-235

New Zealand Department of Statistics (1976). Survey of Household Electricity Consumption 19711972, Wellington.

Neuberger, M., Rabczenko, D., Moshammer, H. (2007). Extended effects of air pollution on cardiopulmonary mortality in Vienna. Atmospheric Environment, Vol. 41, No. 38: 8549-8556.

NIWA (2006) Climate change scenarios for New Zealand. http:/www.niwa.cri.nz/ncc/clivar/scenarios. Accessed 05/11/07.

O'Dea, D., Tucker, S. (2005). The Cost of Suicide to Society. Wellington: Ministry of Health. http://www.moh.govt.nz/moh.nsf/0/BFA96AA9486D2C3BCC2570D4008009E6/\$File/thecostofsuicid etosociety.pdf. Accessed 04/12/07.

Oreszczyn, T., Sung H, Ridley, I Wilkinson, P. (2006) Determinants of winter indoor temperatures in low income households in England. Energy and Buildings 38 245-252 
Peat, J.K., Dickerson, J., Li, J. (1998). Effect of damp and mould in the home on respiratory health: a review of the literature. Allergy. 53: 120-128.

Pell, J.P., Cobbe, S.M. (1999). Seasonal variations in coronary heart disease. Q. J. Medicine, 92: 689 696.

Pilotto, L., Nitschke, M., Smoth, B., Pisaniello, D., Ruffin, R., McElroy, H., et al. (2003). Randomized controlled trial of unflued gas heater replacement on respiratory health of asthmatic children. Int $\mathrm{J}$ Epidemiol, 33(1), 208-214.

PHARMAC (2007).New Zealand Pharmaceutical Schedule. http://www pharmac.govt.nz/pdf/Sched.pdf. Accessed 05/02/08.

Rankine, J. (2005). Housing and Health in Auckland: A summary of Selected Research. Auckland Regional Public Health Service, Auckland District Health Board: Auckland New Zealand.

Richards, S. (2007). Occasional papers in Housing, No 3: Housing needs of the future .Nelson Tasman Housing Trust

Richardson, G. , , Barton, A., Basham, M, Foy, C., Eick, S.A., Somerville, M. and on behalf of the Torbay Healthy Housing Group, Torquay, UK (2006) The Watcombe housing study: The short-term effect of improving housing conditions on the indoor environment. Science of the Total Environment, Vol. 361, No. 1-3; 73-80

Rose, D. (2007). Comparing Time Present with Time Future: Discounting in the Stern Review. Policy Quarterly, Vol. 3, No.1.

Sanne, C. (2000) Dealing with environmental savings in a dynamical economy - how to stop chasing your tail is the pursuit of sustainability. Energy Policy, 28: 487-495

Scott, G., Scott, H., Turley, M., Baker, M. Economic cost of community-acquired pneumonia in New Zealand adults. New Zealand Medical Journal. Vol. 117, No. 1196.

Shaw, R.A., Crane, J., O'Donnell, T.V., et al. Increasing asthma prevalence in a rural New Zealand adolescent population: 1975-89. Arch Dis Child 1990; 65: 1319-23

Shortt and Rugkasa (2007). "The walls were so damp and cold" fuel poverty and ill health in Northern Ireland: results from a housing intervention. Health \& Place, 13: 99-110.

Sinclair, G. (2006). Price of Kyoto compliant emission units. Report for the New Zealand Treasury.

Somerville, M. Basham, M., Foy, C., Gay, T., Shute, P., Barton, A. (2002) Housing Improvements and Health: The results of a randomized trial - the Watcombe Housing Study. Health Expectations, 5: 127135.

Statistics New Zealand. Producer Price Index.. www.statistics.govt.nz

Statistics New Zealand (2005). National Family and Household Projections 2001(base)-2021 Update. http:/www.stats.govt.nz/NR/rdonlyres/B855DF8A-71B4-4D4A-9D4E-

1B7E1 1375106/0/nationalfamilyhouseholdprojections01base21updatehotp.pdf. Accessed 03/08/07.

Statistics New Zealand (2006). Quarterly Employment Survey, June 2006. http://www.stats.govt.nz/NR/rdonlyres/OC5A177C-AA5D-4A36-BC89. I61F87500A1F/15393/qesjun06qtralltables xls. Accessed 02/02/08.

Statistics New Zealand (2007). QuickStats About Housing (Revised 2). http:/www.stats.govt.nz/NR/rdonlyres/2471D004-399D-472D-95DA9020B30C 9386/0/quickstatsabouthousingrevised2.pdf Accessed 28-12-07. 
Statistics New Zealand (2007A) Average hourly earnings.

http://www.stats.govt.nz/NR/rdonlyres/228EA244-5E3C-443A-9F9D-7129AE279FE9/0/alltabs.xls. Accessed 06/09/07.

Stern, N. (2007). The Economics of Climate Change: The Stern Review. University Press, Cambridge.

Taras, H., Potts-Datema, W. (2005). Childhood Asthma and Student Performance at School. Journal of School Health. Vol. 75, 8: 296-312.

Thomson, H., Petticrew, M., Morrison, D. (2002). Housing Improvement and Health Gain: A summary and systematic review. MRC Social \& Public Health Sciences Unit. Occasional Paper No. 5.

Tol, R.J.S. The marginal damage costs of carbon dioxide emissions: an assessment of the uncertainties. Energy Policy, Vol. 33, No. 16: 2064-2074.

Treasury (2005). Cost benefit Analysis Primer. New Zealand Treasury. Wellington. http://www.treasury.govt.nz/publications/guidance/costbenefitanalysis. Accessed 06-10-07.

Tunnicliffe W, B. P., Ayres J, . (1994). Effect of domestic concentrations of nitrogen dioxide on airway responses to inhaled allergen in asthmatic patients. The Lancet, 344: 1733-1736

U.S. EPA (1999). Cost of Illness Handbook. Part IV: Respiratory Illnesses. Chapter IV .2. Cost of Asthma. http://www.epa.gov/oppt/coi/pubs/IV_2.pdf. Accessed 10/10/07.

Verbeeck, G., Hens, H. Energy savings in retrofitted dwellings: economically viable? (2005). Energy and Buildings. 37: 747-754.

Walker, R. (2004) Heat Pump Survey. Community Energy Action, Christchurch.

Walker, J.J., Mitchell, R., Platt, S.D., Petticrew, M.P., Hopton, J. (2006). Does usage of domestic heating influence internal environmental conditions and health. European Journal of Public Health, Vol. 16, 5: 463-469.

Weich, S., Lewis, G. (1998). Material standard of living, social class and the prevalence of common mental disorders in Great Britain. Journal of Epidemiology and Community Health. 52: 8-14.

Wilson, N., Howden-Chapman, P., Crane, J., Wickens, K., Chapman, R. (2007). Potential Health Impacts Associated with Mould in "Leaky Buildings": A review commissioned by the Auckland City Council.

World Health Organization (1987). Health impact of low indoor temperatures: Report on a WHO meeting, Copenhagen,11-14 November, 1985. Copenhagen.

World Health Organization MONICA Project Principal Investigators (1988). The World Health Organization MONICA Project (Monitoring trends and determinants in cardiovascular disease): a major international collaboration. Journal of Clinical Epidemiology 41(2): 10514.

Young, L. (2002). Treasury Working Paper 02/21: Determining the Discount Rate for Government Projects. Wellington: The Treasury. 